\title{
Rubidium-Strontium Geochronology and Plate-Tectonic Evolution of the Southern Part of the Arabian Shield
}

GEOLOGICAL SURVEY PROFESIONAL PAPER 1131

Prepared in cooperation with the Saudi Arabian Ministry of Petroleum and Mineral Resources, Directorate General of Mineral Resources

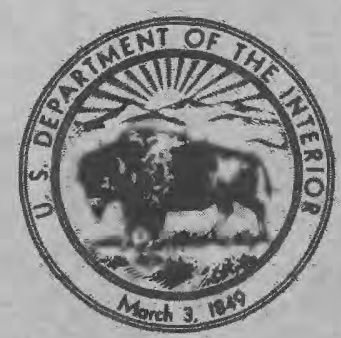




\section{Rubidium-Strontium Geochronology and Plate-Tectonic Evolution of the Southern Part of the Arabian Shield}

By ROBERT J. FLECK, WILLIAM R. GREENWOOD, DONALD G. HADLEY, R. ERNEST ANDERSON, and DWIGHT L. SCHMIDT

Prepared in cooperation with the Saudi Arabian

Ministry of Petroleum and Mineral Resources,

Directorate General of Mineral Resources

A study of the age of rocks and

the geologic history of

southwestern Saudi Arabia

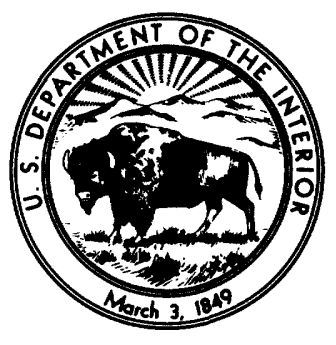




\section{UNITED STATES DEPARTMENT OF THE INTERIOR}

\section{CECIL D. ANDRUS, Secretary}

\section{GEOLOGICAL SURVEY}

H. WiHiam Menard, Director

Library of Congress Cataloging in Publication Data

United States. Geological Survey.

Rubidium-strontium geochronology and plate-tectonic evolution of the southern part of the Arabian Shield.

(Geological Survey professional paper ; 1131)

Bibliography: p.

1. Rubidium-strontium dating. 2. Plate tectonics. 3. Geology-Arabia. I. Fleck, Robert J. II. Title. III. Series: United States. Geological Survey.

Professional paper ; 1131.

QE508.U5 $1979 \quad 555.3 \quad 79-13596$

For sale by the Superintendent of Documents, U.S. Government Printing Office

Washington, D.C. 20402 


\section{CONTENTS}

Abstract

Introduction

Acknowledgments

General geologic setting -

Volcanic and sedimentary rocks ...............

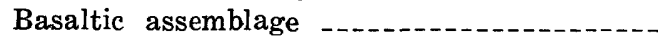

Andesitic assemblage

Plutonic rocks

Dioritic batholiths

Granodiorite gneiss _.......................

Late-orogenic or postorogenic plutons

Layered gabbro and associated rocks ....

Granodiorite to granite intrusions ......

Najd fault system

Previous geochronologic studies

Analytical techniques

Results of rubidium-strontium analyses

Basaltic assemblage
Page

1

1

1

3

3

3

5

5

5

6

7

Results of rubidium-strontium analyses-Continued

Undisturbed isotopic systems _-_._-_-- 16

Disturbed isotopic systems _._._._._._.-. 17

Foliated diorite to trondhjemite batholiths _...- 19

Granodiorite gneiss domes _................. 22

Late-orogenic or postorogenic granodiorite to

granite plutons -..-.- 24

Tindahah batholith _.....-. 24

Bani Thuwr pluton -..-_-_- 24

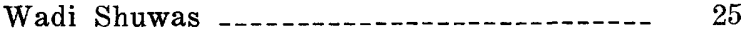

Jabal Shada and Jabal Ibrahim _..._._._.-. 25

Jabal Qal and Jabal 'Aya _...........--- 25

Wadi Halal _-

Wadi al Miyah and Wadi Musayrah _...-- 26

Other late-orogenic or postorogenic plutons - 28

Discussion of the results

Conclusions -

References cited _... 36

\section{ILLUSTRATIONS}

1. Map of the western part of the Arabian Peninsula showing the area studied and the exposed part of the Arabian Shield

2. Chart showing a generalized chronology of igneous and sedimentary units of the southern part of the Arabian Shield and of tectonic events affecting them

3. Geologic map of the southern part of the Arabian Shield in the Kingdom of Saudi Arabia

4. Index map of the location of analyzed samples from the Arabian Shield and of quadrangles -...5-25. Total-rock rubidium-strontium isochron diagrams:

5. Basalt of Wadi al Faqh

6. Arphan Formation of Hadley and meta-andesite of the Junaynah quadrangle

7. Khadrah Formation metavolcanic rocks, rhyolite of the Bi'r Juqjuq quadrangle, and Arphan Formation of Hadley

8. Metavolcanic rocks of Wadi bin Dwaynah

9. Metavolcanic rocks of Hishat al Hawi and Wadi Shuklalah

10. Rhyolite of the Murdama Group

11. Juqjuq Formation of Hadley

12. Quartz diorites of Wadi Khadrah and Wadi ash Shaqah ash Shamiyah, Al Lith area, and of Biljurshi and Wadi Qanunah, Biljurshi area

13. Quartz diorites of An Nimas, Wadi Tarj, and Al Mushirah and the trondhjemite of Wadi Asmak, An Nimas batholith

14. Quartz diorites of Wadi Makhdhul and Simlal and the diorite and trondhjemite of the Wadi Tarib batholith, Wadi Malahah quadrangle

15. Quartz diorites of Jabal Umm al Hashiyah and the southwestern Al Qarah quadrangle -..-

16. Quartz diorite of Malahah dome and granodiorite of Wadi Malahah

17. Granodiorite gneiss of Jabal Mina _.

18. Granodiorite gneiss of Wadi Bagarah and granitic gneiss of Jiddah Airport 
Figures 5-25. Total-rock rubidium-strontium isochron diagrams-Continued

19. Granitic gneisses of Harisi dome and Wadi Bishah

20. Tindahah batholith and the Bani Thuwr pluton, quartz monzonites of Wadi Shuwas, and granites of Jabal Shada and Jabal Ibrahim -

21. Quartz monzonites of Jabal Qal and Jabal 'Aya _.

22. Granodiorite of Wadi Halal

23. Granodiorites of Wadi al Miyah and Wadi Musayrah and an aplite dike of the southwestern Al Qarah quadrangle

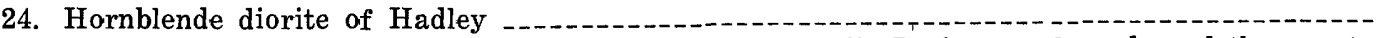

25. Quartz monzonite and gneissic granodiorite of the Bi'r Juqjuq quadrangle and the quartz monzonite of Jabal Tarban

26. Diagrams showing variation of ${ }^{87} \mathrm{Sr} /{ }^{86} \mathrm{Sr}$ and of $\mathrm{Rb} / \mathrm{Sr}$ with age 27, 28. Sketch maps showing geographic distribution of ages:

27. Dioritic complexes and metavolcanic rocks older than 680 million years

28. Volcanic rocks and late-orogenic or postorogenic plutonic rocks younger than 680 million years -

29. Diagram of a plate-tectonic model of the origin and deformation of the Arabian Shield .......

30. Map showing a comparison of continental-collision-related structures of Asia with orogenic structures of the Arabian Shield

TABLES

TABLE

1. Analytical results for rocks of the Arabian Shield

2. Summary of Rb-Sr ages and isochron data

\section{CONVERSION FACTORS}

\begin{tabular}{|c|c|c|c|c|c|c|}
\hline Metric unit & \multicolumn{3}{|c|}{ Inch-Pound equivalent } & \multicolumn{3}{|c|}{ Inch-Pound equivalent } \\
\hline \multicolumn{4}{|c|}{ Length } & \multicolumn{3}{|c|}{ Specific combinations-Continued } \\
\hline $\begin{array}{l}\text { millimeter }(\mathrm{mm}) \\
\text { meter }(\mathrm{m})(\mathrm{km}) \\
\text { kilometer }(\mathrm{km}) \\
\end{array}$ & $\begin{array}{l}\equiv \\
\equiv \\
\equiv\end{array}$ & $\begin{array}{l}0.03937 \\
3.28 \\
.62 \\
\end{array}$ & $\begin{array}{l}\text { inch }(\mathrm{in}) \\
\text { feet }(\mathrm{ft}) \\
\text { mile }(\mathrm{mi})\end{array}$ & $\begin{array}{l}\text { liter per second }(\mathrm{L} / \mathrm{s}) \\
\text { cubic meter per second } \\
\text { per square kilometer } \\
{\left[\left(\mathrm{m}^{3} / \mathrm{s}\right) / \mathrm{km}^{2}\right]}\end{array}$ & $\equiv 91.0353$ & $\begin{array}{l}\text { cubic foot per second } \\
\text { cubic feet per second per } \\
\text { square mile }\left[\left(\mathrm{ft}^{3} / \mathrm{s}\right) / \mathrm{ml}^{2}\right]\end{array}$ \\
\hline \multicolumn{4}{|c|}{ Area } & meter per day $(\mathrm{m} / \mathrm{d})$ & $=3.28$ & $\begin{array}{l}\text { feet per day (hydraulic } \\
\text { conductivity) (ft/d) }\end{array}$ \\
\hline $\begin{array}{l}\text { square meter }\left(\mathrm{m}^{2}\right) \\
\text { square kilometer }\left(\mathrm{km}^{2}\right) \\
\text { hectare (ha) }\end{array}$ & & $\begin{array}{c}10.76 \\
.386 \\
2.47 \\
\end{array}$ & $\begin{array}{l}\text { square feet }\left(\mathrm{ft}^{2}\right) \\
\text { square mile }\left(\mathrm{mi}^{2}\right) \\
\text { acres }\end{array}$ & $\begin{array}{l}\text { meter per kilometer } \\
(\mathrm{m} / \mathrm{km}) \\
\text { kilometer per hour }\end{array}$ & $\begin{array}{l}=5.28 \\
=.9113\end{array}$ & $\begin{array}{l}\text { feet per mile }(\mathrm{ft} / \mathrm{mi}) \\
\text { foot per second }(\mathrm{ft} / \mathrm{s})\end{array}$ \\
\hline \multicolumn{4}{|c|}{ Volume } & $\begin{array}{l}(\mathrm{km} / \mathrm{h}) \\
\text { meter per second }(\mathrm{m} / \mathrm{s})\end{array}$ & $=3.28$ & \\
\hline $\begin{array}{l}\text { cubic centimeter }\left(\mathrm{cm}^{3}\right) \\
\text { liter }(\mathrm{L})\end{array}$ & & $\begin{array}{l}0.061 \\
61.03\end{array}$ & $\begin{array}{l}\left.\text { cubic inch (in }{ }^{3}\right) \\
\text { cubic inches }\end{array}$ & $\begin{array}{l}\text { meter squared per day } \\
\left(\mathrm{m}^{2} / \mathrm{d}\right)\end{array}$ & $=10.764$ & $\begin{array}{l}\text { feet squared per day }\left(\mathrm{ft}^{2} / \mathrm{d}\right) \\
\text { (transmissivity) }\end{array}$ \\
\hline $\begin{array}{l}\text { cubic meter }\left(\mathrm{m}^{3}\right) \\
\text { cubic meter }\end{array}$ & & & $\begin{array}{l}\text { cubic feet }\left(\mathrm{ft}^{3}\right) \\
\text { acre-foot }(\mathrm{acre}-\mathrm{ft})\end{array}$ & $\begin{array}{l}\text { cubic meter per second } \\
\left(\mathrm{m}^{3} / \mathrm{s}\right)\end{array}$ & $=22.826$ & $\begin{array}{l}\text { million gallons per day } \\
(\mathrm{Mgal} / \mathrm{d})\end{array}$ \\
\hline $\begin{array}{l}\text { cubic hectometer }\left(\mathrm{hm}^{3}\right) \\
\text { liter } \\
\text { liter }\end{array}$ & $\begin{array}{l}\equiv 8 \\
\equiv\end{array}$ & $\begin{array}{l}310.7 \\
2.113 \\
1.06\end{array}$ & $\begin{array}{l}\text { acre-feet } \\
\text { pints (pt) } \\
\text { quarts (qt) }\end{array}$ & $\underset{\left(\mathrm{m}^{3} / \mathrm{min}\right)}{\operatorname{cubic} \text { meter }}$ per minute & $=264.2$ & gallons per minute $(\mathrm{gal} / \mathrm{min})$ \\
\hline liter & $\equiv$ & .26 & gallon (gai) & liter per second $(L / s)$ & $=15.85$ & gallons per minute \\
\hline cubic meter & $=$ & .00026 & million gallons (Mgal or & $\begin{array}{l}\text { liter per second per } \\
\text { meter }[(\mathrm{L} / \mathrm{s}) / \mathrm{m}]\end{array}$ & $=4.83$ & $\begin{array}{l}\text { gallons per minute per foot } \\
{[(\mathrm{gal} / \mathrm{min}) / \mathrm{ft}]}\end{array}$ \\
\hline cubic meter & $=$ & 6.290 & barrels (bbl) ( $1 \mathrm{bbl}=42$ gal) & kilometer per hour & .62 & mile per hour $(\mathrm{mi} / \mathrm{h})$ \\
\hline \multicolumn{4}{|c|}{ Weight } & $\begin{array}{l}(\mathrm{km} / \mathrm{h}) \\
\text { meter per second }(\mathrm{m} / \mathrm{s})\end{array}$ & $=2.237$ & \\
\hline $\begin{array}{l}\underset{\text { gram }}{\text { gram }}(\mathrm{g}) \\
\text { metric tons }(\mathrm{t})\end{array}$ & $\equiv$ & $\begin{array}{l}0.035 \\
.0022 \\
1.102\end{array}$ & $\begin{array}{l}\text { ounce, avoirdupois (oz avdp) } \\
\text { pound, avoirdupois (1b avdp) }\end{array}$ & $\begin{array}{l}\text { gram per cubic } \\
\text { centimeter }\left(\mathrm{g} / \mathrm{cm}^{3}\right)\end{array}$ & $=62.43$ & pounds per cubic foot $\left(\mathrm{lb} / \mathrm{ft}^{3}\right)$ \\
\hline $\begin{array}{l}\text { metric tons (t) } \\
\text { metric tons }\end{array}$ & $\equiv$ & $\begin{array}{l}1.102 \\
0.9842 \\
\end{array}$ & $\begin{array}{l}\text { tons, short }(2,000 \mathrm{lb}) \\
\text { ton, long }(2,240 \mathrm{lb})\end{array}$ & $\begin{array}{l}\text { gram per square } \\
\text { centimeter }\left(\mathrm{g} / \mathrm{cm}^{2}\right)\end{array}$ & $=2.048$ & pounds per square foot $\left(\mathrm{lb} / \mathrm{ft}^{2}\right)$ \\
\hline \multicolumn{4}{|c|}{ Specific combinations } & $\begin{array}{c}\text { gram per square } \\
\text { centimeter }\end{array}$ & .0142 & pound per square inch $\left(\mathrm{lb} / \mathrm{in}^{2}\right)$ \\
\hline kilogram per square & & 0.96 & atmosphere (atm) & \multicolumn{3}{|c|}{ Temperature } \\
\hline $\begin{array}{l}\text { kilogram per square } \\
\text { centimeter } \\
\text { cubic meter per second } \\
\left(\mathrm{m}^{3} / \mathrm{s}\right)\end{array}$ & & $\begin{array}{l}.98 \\
35.3\end{array}$ & $\begin{array}{l}\text { bar }(0.9869 \mathrm{~atm}) \\
\text { cubic feet per second }\left(\mathrm{ft}^{3} / \mathrm{s}\right)\end{array}$ & $\begin{array}{l}\text { degree Celsius }\left({ }^{\circ} \mathrm{C}\right) \\
\text { degrees Celsius } \\
\text { (temperature) }\end{array}$ & $\begin{array}{l}=1.8 \\
=\left[\left(1.8 \times{ }^{\circ} \mathrm{C}\right.\right.\end{array}$ & $\begin{array}{l}\text { degrees Fahrenheit }\left({ }^{\circ} \mathbf{F}\right) \\
+32 \text { ] degrees Fahrenheit }\end{array}$ \\
\hline
\end{tabular}




\title{
RUBIDIUM-STRONTIUM GEOCHRONOLOGY AND PLATE-TECTONIC EVOLUTION OF THE SOUTHERN PART OF THE ARABIAN SHIELD
}

\author{
By Robert J. Fleck, William R. Greenwood, Donald G. Hadley, \\ R. Ernest Anderson, and Dwight L. Schmidt
}

\begin{abstract}
Rubidium-strontium studies of Precambrian volcanic and plutonic rocks of the Arabian Shield document an early development of the Arabian craton between 900 and 680 m.y. (million years) ago. Geologic studies indicate an islandarc environment characterized by andesitic (dioritic) magmas, volcaniclastic sedimentation, rapid deposition, and contemporaneous deformation along north- or northwest-trending axes. Magmatic trends show consistent variation in both composition and geographic location as a function of age. The oldest units belong to an assemblage of basaltic strata exposed in western Saudi Arabia that yield an age of $1165 \pm 110$ m.y. The oldest andesitic strata studied yield an age of $912 \pm 76 \mathrm{~m} . \mathrm{y}$. The earliest plutonic units are diorite to trondhjemite batholiths that range from 800 to 900 m.y. in age and occur along the western and southern parts of Saudi Arabia. Younger plutonic units, 680 to $750 \mathrm{~m} . \mathrm{y}$. in age, range from quartz diorite to granodiorite and become more abundant in the central and northeastern parts of the Arabian Shield. Initial ${ }^{\mathrm{s}} \mathrm{Sr} /{ }^{\mathrm{se}} \mathrm{Sr}$ ratios for both dioritic groups range from 0.7023 to 0.7030 and average 0.7027 . The absence of sialic detritus in sedimentary units and the evidence for an island-arc environment suggest the early development of the Arabian craton at a convergent plate margin between plates of oceanic lithosphere. Active subduction apparently extended from at least 900 m.y. to about 680 m.y.
\end{abstract}

Subsequent to this subduction-related magmatism and tectonism, called the Hijaz tectonic cycle, the Arabian craton was sutured to the late Precambrian African plate in a collisional event. This period of orogeny, represented in Arabia and eastern Africa by the Mozambiquian or PanAfrican event, extended from some time before $650 \mathrm{~m} . \mathrm{y}$. to at least $540 \mathrm{~m} . \mathrm{y}$. and perhaps $520 \mathrm{~m} . \mathrm{y}$. B.P. Although the tectonic processes of subduction and continental collision during the $900+$ to $500-$ m.y. period require similar directions of plate convergenoe, the differences in magmatic and tectonic styles of Hijaz orogenesis from those of the PanAfrican and the temporal break between them in much of the southern part of the Arabian Shield support division into at least two events. As defined by the ages of major plutonic units, the axis of magmatic and tectonic activity migrated eastward or northeastward during the Hijaz cycle, the predominantly dioritic plutonic rocks becoming younger and more siliceous to the east. Granodiorite to granite plutonism of the Pan-African event, however, shows no geographic bias, being distributed throughout the Arabian Shield. Although the Hijaz diorites and Pan-African granitic rocks exhibit strong contrasts in composition and age differences as great as $250 \mathrm{~m} . \mathrm{y}$. in the westernmost parts of the area, the two groups are less distinct compositionally and nearly the same age in the eastern part.

\section{INTRODUCTION}

Geologic and geochronologic studies of the southern part of the Arabian Shield present a complex but surprisingly clear view of late Precambrian island-arc development and crustal evolution. The geology of the Arabian Shield between lat $21^{\circ} 30^{\prime} \mathrm{N}$. and the Saudi Arabian-Yemen border (fig. 1) is discussed by Schmidt and others (1973) and by Greenwood and others $(1973,1976,1977)$; geologic mapping was compiled by Greenwood and others (1974). Geochronologic studies of the shield include those by Fleck and others (1972, 1973, and 1976). Results of geologic, geochemical, and preliminary geochronologic studies have been interpreted as indicating formation of the Arabian Shield in an intraoceanic island arc by andesitic volcanism, associated plutonism, and cannibalistic sedimentation (Greenwood and others, 1976, 1977). This report presents $\mathrm{Rb}-\mathrm{Sr}$ results that document a detailed chronology for the development of the shield area. These results were obtained by the U.S. Geological Survey as part of the program of geologic investigations of the Directorate General of Mineral Resources, Ministry of Petroleum and Mineral Resources, Kingdom of Saudi Arabia.

\section{ACKNOWLEDGMENTS}

We thank A. Berry, J. Saburomaru, and W. Doering for laboratory assistance with the rubidiumstrontium analyses reported here. We also thank T. H. Kiilsgaard, W. C. Prinz, R. G. Coleman, J. C. Ratte, and H. R. Cornwall for assistance of various kinds. M. A. Lanphere and F. C. W. Dodge made many useful suggestions. 


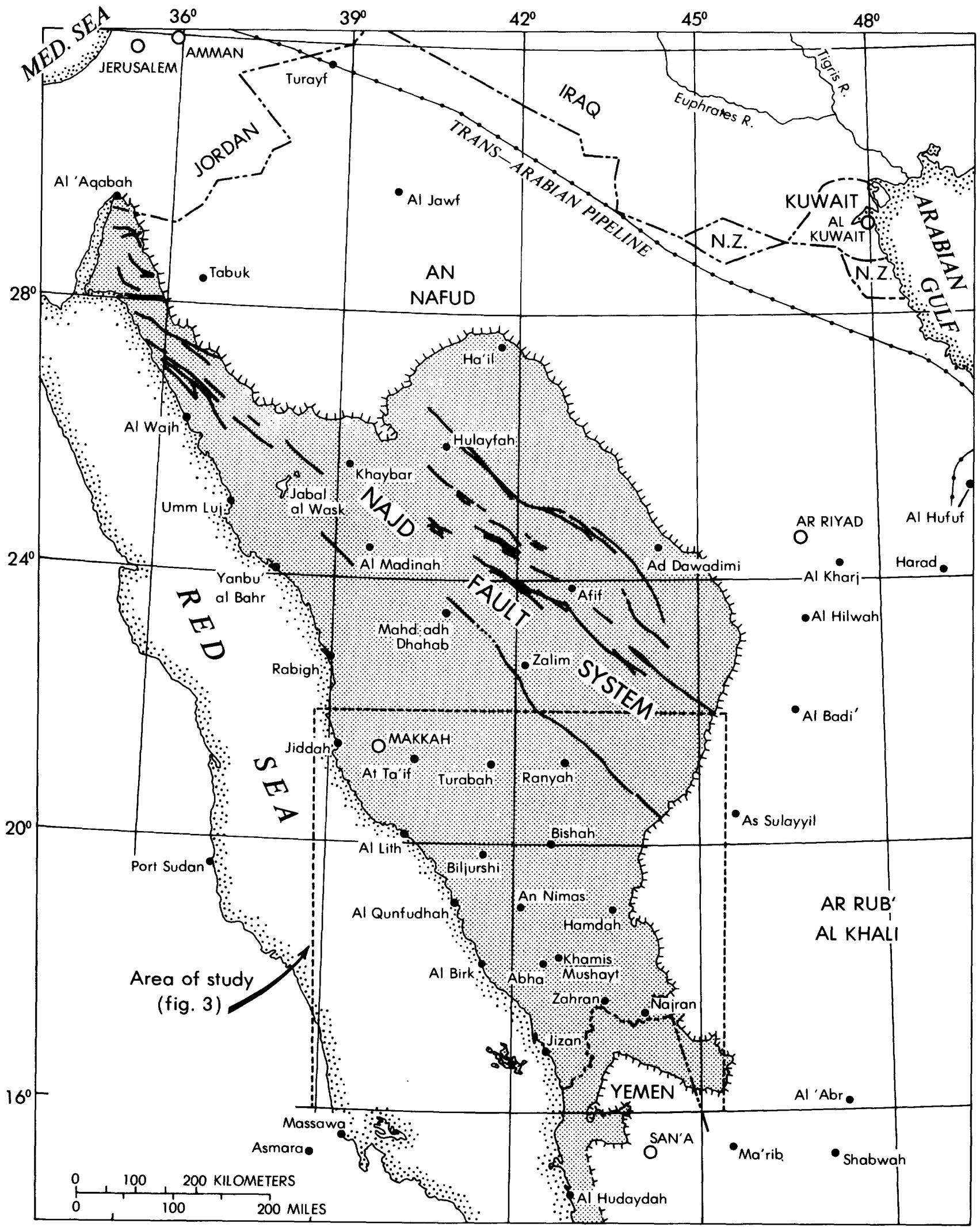

FIGURE 1.-Western part of the Arabian Peninsula showing the area studied and the exposed part of the Arabian Shield (shaded). Hachures are on Paleozoic side of contact between Paleozoic units and units of the shield. Only representative major faults of the Najd fault system are shown. 


\section{GENERAL GEOLOGIC SETTING}

The Arabian Shield represents a terrane of slightly to intensely deformed stratified units and undeformed to partly remobilized plutonic units of late Precambrian (Proterozoic) age. The shield is exposed over an area of $610,000 \mathrm{~km}^{2}$ in the western part of the Arabian Peninsula (fig. 1). On the north and east the shield is overlain by a veneer of nearly flat-lying Phanerozoic sedimentary rocks. On the west the Red Sea rift, containing "oceanic" or "basaltic" crust of Tertiary and Quaternary age, separates the Arabian Shield from its previously continuous counterpart, the Nubian (or African) Shield in Egypt, Sudan, and Ethiopia. A summary of the major sedimentary and igneous units of the southern part of the shield and a generalization of the type of tectonic environment are shown in figure 2. The distribution of these units in the area studied is shown on the geologic map (fig. 3), which is modified from Greenwood and others (1974).

\section{VOLCANIC AND SEDIMENTARY ROCKS BASALTIC ASSEMBLAGE}

The oldest rocks exposed in the southern part of the Arabian Shield constitute an assemblage of clastic and volcaniclastic marine sedimentary rocks, basalt and basaltic andesite flows, flow breccias, tuffs, and, in lesser amounts, chert and impure limestone (marble). Sedimentary units exhibit graded bedding, slump structures, intraformational breccias,

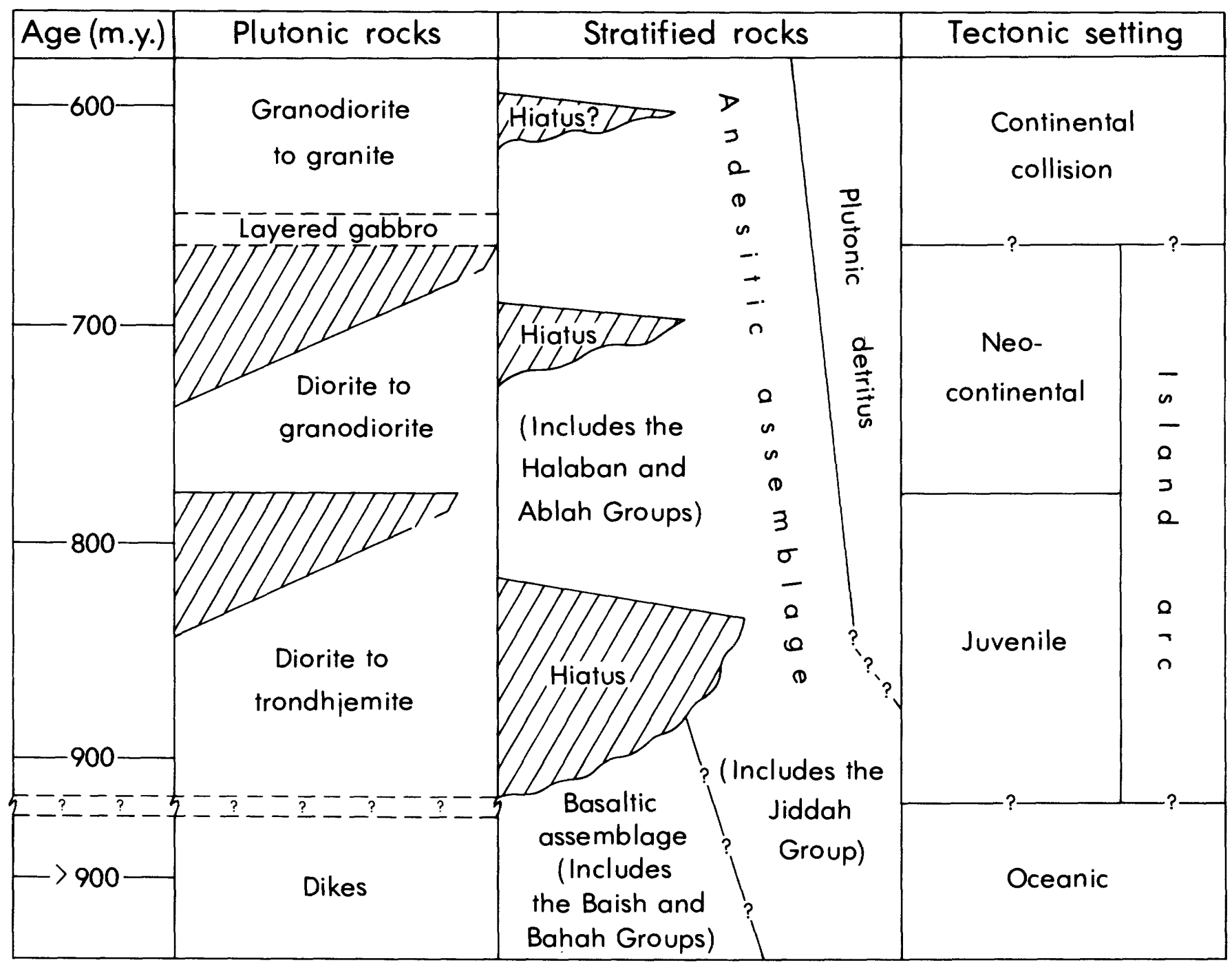

FIGURE 2.-Generalized chronology of igneous and sedimentary units of the southern part of the Arabian Shield and of tectonic events affecting them. Periods indicated by shading were of decreased igneous activity or deposition and are shown as time transgressive because of a general southwest to northeast decrease in age of the early shield-forming units. 


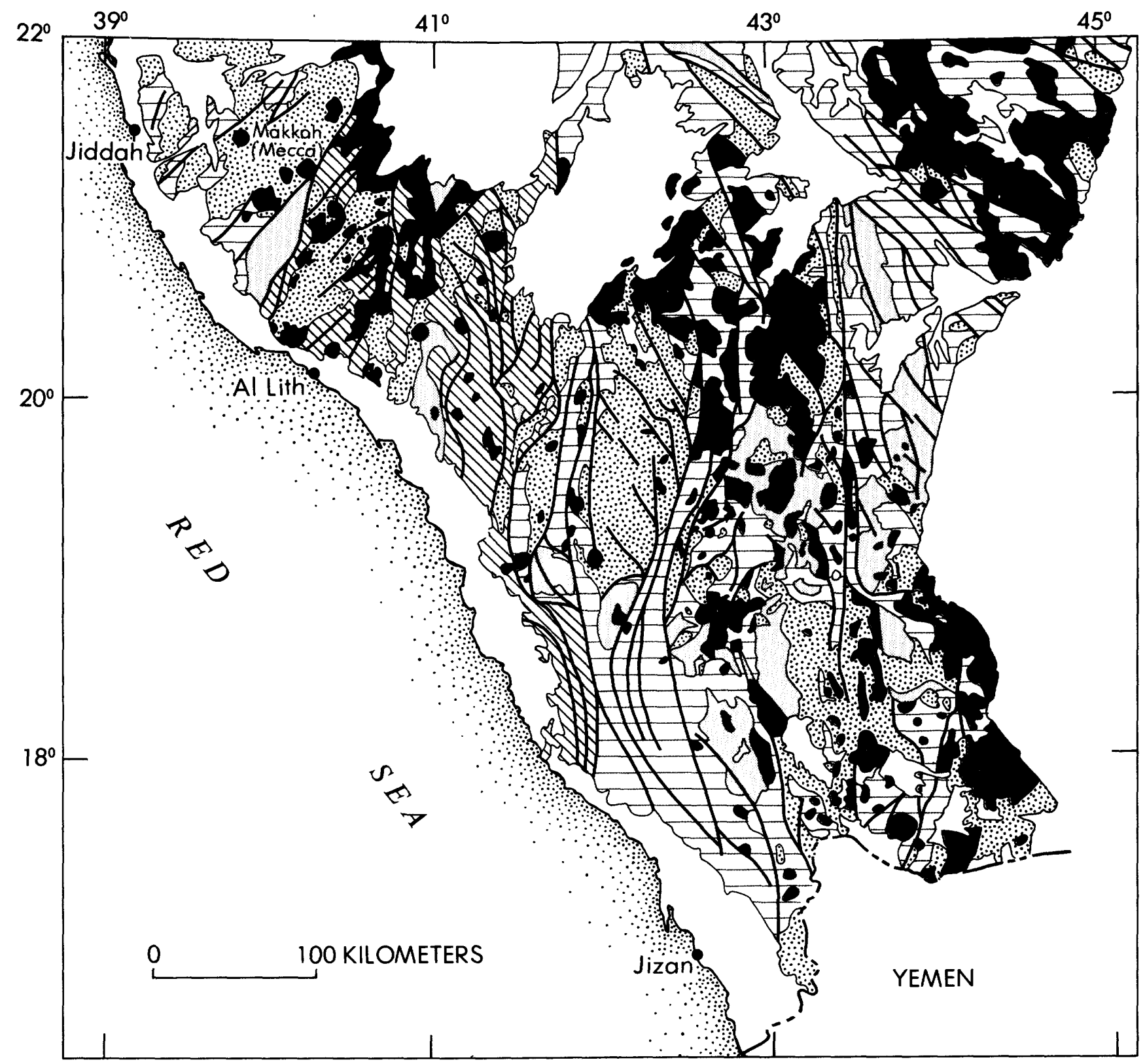

EXPLANATION

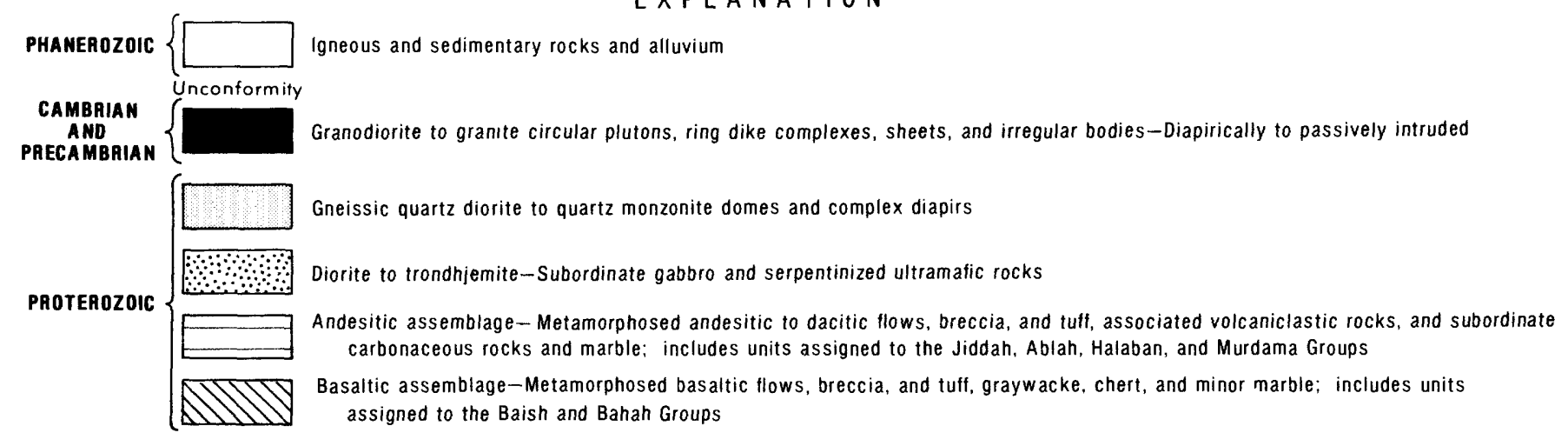

Figure 3.-Geologic map of the southern part of the Arabian Shield in the Kingdom of Saudi Arabia. Modified from Greenwood and others (1974). 
and similar features characteristic of turbidites. Algal structures have been identified in some carbonaceous units (T. Kiilsgaard, oral commun., 1977). Neither potassium feldspar clasts nor terrigenous lithic fragments have been reported from these strata. Contacts between this "basaltic assemblage," as we shall refer to these units, and any other rock unit within the area studied are either tectonic or indicate this unit to be the older. No evidence has been found within the southern part of the Arabian Shield to suggest deposition of the basaltic assemblage on sialic crust, and no older rock units are known. The basaltic assemblage includes units assigned by various workers to the Baish and Bahah Groups. Bakor and others (1976) identify a maficultramafic complex at Jabal al Wask in the northern part of the shield (fig. 1) as "back-arc ophiolite." Although serpentinite occurs within the southern part of the shield, no relation to rocks of the basaltic assemblage has been demonstrated. The sedimentary aspects of the "ophiolite" described by Bakor and others are similar to those described here, but none of the volcanic rocks studied in the southern shield could be classified as spilitic. Any relation between this "ophiolite" and the basaltic assemblage, however, remains to be demonstrated.

\section{ANDESITIC ASSEMBLAGE}

The stratified units of the southern part of the Arabian Shield not part of the basaltic assemblage are classified here as the "andesitic assemblage." This generalization of previously published stratigraphic nomenclature is necessary for several reasons. Correlation of units of an extremely thick, laterally variable, highly deformed, and slightly to highly metamorphosed sequence of lithologically repetitious strata over $610,000 \mathrm{~km}^{2}$ must be described as difficult at best. This remains true when the study area is restricted to the southern one-third of the shield area. Available age determinations are inadequate for regional correlation of strata at a formational level and probably questionable at the group level. Alteration, sedimentary reworking, and varying degrees of metamorphism further complicate the geochronologic interpretation and, as will be shown, invalidate many of the age determinations made on fine-grained volcanic or volcaniclastic units. The andesitic assemblage consists predominantly of calcalkalic andesite, basaltic andesite, dacite, associated volcaniclastic rocks, and subordinate carbonaceous rocks and marble. The volcanic rocks include flows, breccias, and large amounts of tuff, including welded ash-flow tuff. Locally, younger units of this assem- blage rest unconformably on older units of the assemblage, on older plutonic rocks, or on the basaltic assemblage. In some localities, the younger units contain dioritic to granitic detritus; in other areas, the rocks cannot be subdivided easily on this basis.

The relation between the oldest units of the andesitic assemblage and the basaltic assemblage is uncertain because all contacts between these two groups of strata are tectonic. Because the two assemblages are chemically and lithologically distinct, never occur in the same tectonic block, and contain units whose characteristics require significantly different environments of deposition, we conclude that these assemblages accumulated at different times or in areas originally remote one from another and were juxtaposed subsequently by faulting along northsouth shear zones. Although the nature of this displacement is inadequately known, it appears to have occurred during deposition of the andesitic assemblage and therefore must be consistent with the island-arc origin of that sequence.

\section{PLUTONIC ROCKS}

\section{DIORITIC BATHOLITHS}

The basaltic assemblage and the older units of the andesitic assemblage are complexly deformed, metamorphosed, and intruded by diorite, quartz diorite, and trondhjemite batholithic complexes. The intrusive sequence within these complexes generally follows a differentiation trend, beginning with hornblende diorite and gabbro followed by quartz diorite and trondhjemite. In places, however, the sequence was repeated, the trondhjemite being intruded by younger quartz diorite or hornblende diorite. Chemical variations within the batholiths include ranges in $\mathrm{SiO}_{2}$ from 55 to 73 percent, $\mathrm{K}_{2} \mathrm{O}$ from less than 0.4 to more than 1.7 percent, and $\mathrm{CaO}$ from less than 2 to more than 8.5 percent (Greenwood and Brown, 1973). Normative orthoclase commonly makes up less than 10 percent, especially in the western part of the area. As are the older strata, the diorite batholiths are cut by zones of intense shearing that segment the bodies into narrow north-trending slices, at many places separated by similarly sheared blocks of stratified units. Unlike the two older stratified assemblages, which never occur in the same tectonic slices, the batholiths retain a general coherence and, though cut by the same shear zones, exhibit no significant lateral displacement. This relation suggests that batholithic intrusion was probably concurrent with much of the deformation but that the major translation required to juxtapose the older 
units of the andesitic assemblage with the basaltic terrane occurred earlier.

Diorite-trondhjemite complexes (fig. 3) in the southwesternmost part of the shield have been assigned to three large batholiths-Biljurshi, An Nimas, and Wadi Tarib-that exhibit an en echelon pattern from northwest to southeast. The northwesternmost complex, the Biljurshi batholith (Greenwood, 1975a), includes dioritic bodies between $\mathrm{Al}$ Lith and the major north-trending shear zone at approximately long $41^{\circ} 45^{\prime} \mathrm{E}$. South and east of the Biljurshi batholith, dioritic units are assigned to the An Nimas batholith (Greenwood, 1979a; Anderson, 1977), which extends north from the town of An Nimas between long $41^{\circ} 45^{\prime}$ and long $42^{\circ} 30^{\prime} \mathrm{E}$. Dioritic intrusive rocks south and east of the An Nimas batholith are assigned to the Wadi Tarib batholith, which occupies a broad area in the southeastern part of the Arabian Shield (Greenwood, $1979 \mathrm{c}, \mathrm{d})$. The Wadi Tarib batholith includes both dioritic and granodioritic units mapped previously as Khamis Mushayt Gneiss (Coleman, 1973a).

Northeast of the en echelon batholiths, exposures of diorite are generally less extensive, as younger andesitic units cover and younger granodiorite to granite bodies intrude the older terrane. East of the town of Bishah, discontinuous exposures suggest that a large dioritic batholith may once have occupied a large area but this has been reduced by emplacement of a younger granitic body, the granodiorite of Wadi Musayrah.

Subsequent to emplacement of the early dioritic batholiths, younger units of the andesitic assemblage, including conglomerates containing diorite and trondhjemite cobbles, were deposited on both older volcanic assemblages and on erosionally exposed plutons of the batholiths themselves. The younger units of the andesitic assemblage indicate a continuation of andesitic to dacitic volcanism and deposition of andesitic volcaniclastic, fine-grained terrigenous, and carbonate sediments, but the plutonic detritus in the abundant breccias and intraformational conglomerates indicates local uplift and erosion of the early dioritic bodies. Because the younger andesitic strata were deposited on both older andesitic units and the basaltic assemblage, juxtaposition of these two older sequences must have been largely complete before that time. Because these younger andesitic units are also displaced by the north-south shear zones and range in metamorphic grade up to almandine-amphibolite facies (including kyanite and sillimanite in some localities), deformation and metamorphism clearly must have continued for a significant period of time. In some areas, the younger units of the andesitic assemblage are intruded by a second, or younger, generation of diorite batholiths that may in turn be overlain by even younger graywacke, andesite, volcanic tuff, breccia, and agglomerate (fig. 2). Plutonic detritus increases upward in the assemblage but is clearly controlled by local exposures of older diorites. As lateral variations of detrital components are common within these strata, the presence or absence of dioritic detritus at a given locality is unreliable as a means of correlation.

\section{GRANODIORITE GNEISS}

The first major shift in plutonic rock type occurred in the western part of the Arabian Shield after the major dioritic complexes were emplaced in that area. Compositionally variable gneiss domes, ranging from biotite-bearing trondhjemite to biotite-muscovite granite, were emplaced with both internal and host-rock foliations concordant with the intrusive margins. Greenwood (1979a) describes the granodiorite orthogneiss of Jabal Mina as “... a nested to interconnected series of phacoidal and lensoidal bodies separated by thin septae of metamorphic layered rocks. The bodies appear to be regionally conformable with the structure of the metamorphic rocks but have slightly to moderately discordant contacts when viewed on an outcrop scale." Gneiss domes of this general description, though not abundant, are common in the southwestern part of the Arabian Shield. The rocks range from quartz diorite to quartz monzonite and generally contain both biotite and muscovite but no hornblende. The fabric of these rocks is strongly planar, defined primarily by oriented micaceous minerals and stretched inclusions. This foliation is commonly antiformal or domical, paralleling that of the deformed metamorphic rocks at the margins. Contacts between the gneiss domes and adjacent stratified units vary in definition, some being determinable to within a millimeter, as where the intruded strata are amphibolite-grade metavolcanic rocks, and others being almost completely gradational from sillimanite-bearing metapelites into biotite-muscovite granodiorite gneiss. Locally, garnet is an accessory mineral.

The metamorphic grade of stratified units adjacent to these gneiss domes is generally high, and kyanite and sillimanite are common where $\mathrm{Al}_{2} \mathrm{O}_{3}$ contents are adequate for their formation. Grade of metamorphism decreases away from the domes to that of an average greenschist facies, the minimum metamorphic grade of all older strata in this area of the 
Arabian Shield. This metamorphic gradient adjacent to the domes indicates a clear genetic relationship between the gneiss domes and high-pressure, hightemperature metamorphism, but designation of either as cause or effect is generally uncertain.

The gneiss domes are generally elongate, doubly plunging antiforms whose axes and longest dimensions are concordant with the generally north-south trend of schistosity, axes of folds, and faults in the strata intruded. Although the gneiss domes may be bounded by the north-trending shear zones, few are actually truncated by the zones. Minor fractures offset the domes, but in general, most field evidence indicates that emplacement of the domes was simultaneous with movement on the north-south shear zones and with the most intense deformation of both the basaltic and andesitic assemblages. These characteristics have led some workers to refer to the domes as "syntectonic" granites (see, for example, Coleman, 1973a, b).

The emplacement of the gneiss domes marks a significant point in the geologic evolution of the Arabian Shield. Strata younger than the domes may be included within the andesitic assemblage but are generally only broadly folded, except near or in large northwest-trending wrench-fault zones of the Najd fault system (Brown and Jackson, 1960; Delfour, 1970). Metamorphism of post-gneiss-dome units does not exceed the greenschist-facies except in contact metamorphic aureoles around younger, lateorogenic or postorogenic granitic plutons. These youngest units of the andesitic assemblage commonly contain large amounts of plutonic detritus but are included with the assemblage because of the dominance of andesitic to dacitic volcanic and volcaniclastic detritus. The more variable distribution of flow rocks in the post-gneiss-dome strata compared to the older units suggests that volcanic centers were more widely spaced than before, giving rise to broad areas of largely detrital units bounded by areas with interbedded flows and clastic strata with fewer areas of predominantly volcanic units.

\section{LATE-OROGENIC OR POSTOROGENIC PLUTONS}

Volumetrically, the units discussed represent the primary constructional phase of the southern part of the Arabian Shield. These units were emplaced or deposited during a period of active orogenesis, and most are deformed and metamorphosed, exhibiting isoclinal folding, cataclasis, and local migmatization. Early plutonic rocks are less obviously deformed than are stratified units, but they are generally foliated and commonly highly sheared. Late-orogenic or postorogenic units, however, show little evidence of deformation. or metamorphism, although those bodies adjacent to such younger structures as faults of the Najd fault system may exhibit local effects. Two major groups of plutonic rock units occur as late-orogenic or postorogenic bodies: (1) layered gabbro and associated rocks and (2) granodiorite to granite intrusions.

\section{LAYERED GABBRO AND ASSOCIATED ROCKS}

Gabbroic masses occur as mafic phases of the diorite batholiths, as small masses of metagabbro intruding metavolcanic units, and as largely unmetamorphosed, undeformed, subcircular, layered bodies. The first two of these groups are associated with the active orogenic andesite and diorite magmatism of the island arc and probably represent the plutonic equivalents or more mafic differentiates of island-arc basalts. The layered gabbros are younger than the other groups, being intruded after the gneiss domes but before the last thermal events affecting the Arabian Shield. The layering of these gabbros dips inward from the margins, where it is commonly steep, and flattens toward the center (Coleman and others, 1972, 1973). Compositionally, the layering is rhythmic, controlled largely by variations in amount of plagioclase, and represents crystallization and settling from a subalkaline tholeiitic magma.

GRANODIORITE TO GRANITE INTRUSIONS

Late-orogenic or postorogenic granodiorite to granite plutons that yield $\mathrm{K}$-Ar ages between $\mathbf{5 2 0}$ and 620 m.y. (Fleck and others, 1976) intrude the pre-gneiss-dome units and either intrude the youngest andesitic strata or are their plutonic equivalents. These plutons occur throughout the Arabian Shield, representing the last major shield-forming event, which has been equated with the Pan-African event or orogeny of the African continent (Fleck and others, 1976). The intrusions commonly deflect and invade the north-trending shear zones of the shield, at only a few places showing small displacements produced by late movement. The plutons are affected, however, by left-lateral offsets, some of which may be large, along northwest-trending fractures of the Najd fault system and by small, generally rightlateral displacements having an east-west trend that may be conjugate to that of the Najd (Fleck and others, 1976). In composition, the plutons range from granodiorite to granite and follow a calcalkaline differentiation trend (Greenwood and Brown, 1973). 
Although the granodiorite to granite bodies occur as batholiths in some areas, especially in the eastern part of the Arabian Shield, the more common occurrence is as subcircular ring structures or arcuate, lensoidal cone-sheets emplaced between similarly shaped plates of host rock. Many have steeply dipping primary foliations that parallel their margins. Some of these bodies are interpreted as subvolcanic intrusions, whereas cleavage in others suggests diapiric emplacement. With the exception of volumetrically insignificant mafic and siliceous dikes that may cut these plutons, the granodiorite to granite bodies represent the youngest plutonic units of the Arabian Shield.

\section{NAJD FAULT SYSTEM}

Strata younger than the late-orogenic or postorogenic plutons are confined to the northern part of the shield and are generally related to downfaulted areas of the Najd fault system (Hadley, 1974 ; Delfour, 1970). Although volcanic units occur within these strata, the units are dominantly clastic sedimentary rocks derived by erosion from adjacent fault blocks. The Najd fault system trends northwest-southeast across the entire exposure of the Arabian Shield (fig. 1). Brown (1972) suggests a composite left-lateral offset of as much as $240 \mathrm{~km}$ along the system. Only the easternmost part of the area studied by us (fig. 3) is within the Najd fault system.

\section{PREVIOUS GEOCHRONOLOGIC STUDIES}

Most of the previously reported age determinations from the Arabian Shield are either K-Ar analyses or $\mathrm{Rb}-\mathrm{Sr}$ model ages on minerals. The $\mathrm{Rb}-\mathrm{Sr}$ ages were calculated from assumed initial ${ }^{87} \mathrm{Sr} /{ }^{86} \mathrm{Sr}$ ratios. These results provide a reasonably accurate view of the most recent thermal or tectonic event but generally "telescope" older ages toward that event as mineral ages are reset. In this paper the term "apparent age" is used to describe those ages that are analytically correct but are not necessarily representative of the time of formation or "true age" of the unit studied. "Indicated age" is used in a similar sense by some authors. Many workers-Brown and others $(1963 \mathrm{a}, \mathrm{b})$, Bramkamp and others $(1963 \mathrm{a}, \mathrm{b})$, and Jackson and others (1963)-report both K-Ar and $\mathrm{Rb}-\mathrm{Sr}$ apparent ages on 1:500,000 scale maps of the shield. Other age determinations are reported by Coleman and others (1972), Brown $(1970,1972)$, and Lenz and others (1973). Fleck and others (1976) studied rocks from the southern part of the shield in order to investigate the effectiveness of the $\mathrm{K}-\mathrm{Ar}$ technique in age studies of this area.

Because of the obvious inadequacies of $\mathrm{K}-\mathrm{Ar}$ studies in the older units, studies reported here were begun using $\mathrm{Rb}-\mathrm{Sr}$ whole-rock isochrons. Fleck and others (1976) conclude that a pervasive orogenic event with at least two pulses or thermal maxima affected the Arabian Shield between 510 and about 610 m.y. (B.P.) and correlate this event with the Pan-African (Mozambiquian) orogeny or event of eastern Africa. Potassium-argon ages of amphiboles showed that the diorite-granodiorite (here called diorite to trondjhemite) batholiths and the tonalite to granite (here called granodiorite) gneiss domes were at least 765 m.y. old. Preliminary results of the $\mathrm{Rb}-\mathrm{Sr}$ studies (Fleck and others, 1973) suggested ages of $958 \pm 22 \mathrm{~m} . \mathrm{y}$. and $759 \pm 36 \mathrm{~m} . \mathrm{y}$. $\left(\lambda_{\beta}=1.39 \times\right.$ $\left.10^{-11} \mathrm{yr}^{-1}\right)$, respectively, for one of the diorite batholiths and one gneiss dome. Preliminary interpretations of results presented in this report are cited by Greenwood and others $(1976,1977)$, who suggest a general chronology of orogenesis and plate-tectonic evolution of the Arabian Shield from before 960 m.y. to 550 m.y. The present report, presenting Rb-Sr whole-rock isochrons for the major rock groups, modifies that chronology and extends the plate-tectonic model to explain the late-stage orogenesis of the Pan-African event.

\section{ANALYTICAL TECHNIQUES}

Most rock samples collected for $\mathrm{Rb}-\mathrm{Sr}$ analyses were heavier than $10 \mathrm{~kg}$, although locations and access occasionally obviated the collecting of samples of this size. Weathered material was removed. In all cases the volume of material powdered for analysis was greater than that of a cube whose edges were at least 10 times the maximum dimension of any contained mineral grain. In no case, however, was the weight of material used less than $1 \mathrm{~kg}$. In foliated rocks, these dimensions were arbitrarily increased such that the maximum width of mineral bands was used instead of grain size to determine sample size. Most crushed samples averaged $3 \mathrm{~kg}$ or more. This material was crushed so that the entire amount would pass 150 -mesh Tyler sieves. This sieve size was changed to 200 mesh in the latter part of the study. After sieving, the powder was mixed thoroughly and an aliquant of approximately $30 \mathrm{~g}$ was taken for analyses.

Rubidium and strontium concentrations used in this study were measured either by mass-spectrometric isotope dilution or by X-ray fluorescence 
analysis calibrated by standards measured by isotope dilution. For isotope-dilution analyses, standard silicate-digestion $\left(\mathrm{HF}+\mathrm{HClO}_{4}\right)$ and ion-exchange techniques were followed. Because of the use of an ${ }^{87} \mathrm{Rb}$ spike, rubidium and strontium were analyzed in separate splits to permit correction of ${ }^{87} \mathrm{Sr} /{ }^{86} \mathrm{Sr}$ ratios for even very low levels of rubidium. X-ray fluorescence analyses were made using the technique of Norrish and Chappell (1967), modified slightly to correct for short-term instrumental drift. Uncertainties in isotope-dilution analyses were generally less than 1 percent, as determined by replicate measurements. The principal uncertainty appears to have been sample inhomogeneity, as data for both blank runs and spike calibrations indicate errors of less than 0.5 percent (one standard deviation). Standard deviations of isotope-ratio measurements were generally less than 0.0002 , with standard errors of the mean of less than 0.0001 . Uncertainties in concentrations determined by X-ray fluorescence were calculated as the standard deviation of the calibration curve determined on standards analyzed with the unknowns, using three to six standards with each set of unknowns. In most cases, at least one unknown from each data set was analyzed by isotope dilution and included in the calibration curve. Standard deviations varied from less than $1 \mathrm{ppm}$ (part per million) to about $6 \mathrm{ppm}$ for rubidium (unknowns from 1 to $200 \mathrm{ppm}$ ) and from about 2 to $15 \mathrm{ppm}$ for strontium (unknowns from 10 to $1,200 \mathrm{ppm}$ ). Except those for concentrations below $20 \mathrm{ppm}$, these values generally represented uncertainties between 1 and 4 percent (one standard deviation).

Isochron data were calculated using the leastsquares regression technique of York (1969) with provision for correlated errors. No correlation of errors was used, however, because none of the ${ }^{87} \mathrm{Sr} /{ }^{86} \mathrm{Sr}$ values exceeded 1.0. Studies of two-error regression techniques by Brooks and others (1972) found no significant correlation of errors in samples with ${ }^{87} \mathrm{Sr} /{ }^{86} \mathrm{Sr}$ below 1.0 ; their recommendation to use a correlation coefficient of zero in these cases has been followed here. Uncertainties in age and strontium initial-ratio reported in this study represent values calculated by the York (1969) regression technique using estimates of analytical errors and do not reflect the actual fit of data points to, or degree of scatter about, the best-fit line. As shown by York (1969), to incorporate a measure of the actual degree of scatter of the data points about the best line, the uncertainties reported here are multiplied by the index of fit, (SUMS/ $(n-2))^{1 / 2}$, given here simply as "Index." Because Index values are less than 1.0 in all but three of the isochrons in this study, uncertainties reflecting this degree of fit are much lower than those reported. As discussed by Brooks and others (1972), this Index may be used as an indicator of geologic error on the basis of its comparison of the observed dispersion of the data with that calculated from assigned experimental errors. They suggest a cutoff between analytical and geologic error at an Index level of $1.58{ }^{1}$. With the exception of those for two rock units, Index values obtained in this study are consistently below this value, even when geologic error is implied by other evidence. Because the estimated experimental errors in the concentrations of rubidum and strontium are based not on replicate isotope-dilution analyses of similar samples but on regressions of standards for $\mathrm{X}$-ray fluorescence and on only limited numbers of strictly similar duplicates, we use the Index only as a comparative device, not as a quantitative measure of geologic error. As used in this paper, "isochron" refers only to the type of diagram or the best-fit line for a given set of points and does not imply any conclusion regarding presence or absence of geologic error.

The decay constant of ${ }^{87} \mathrm{Rb}$ used here is $\lambda_{\beta}=1.42 \times$ $10^{-11} \mathrm{yr}^{-1}$ (Neumann and Huster, 1976). A rubidium abundance ratio ${ }^{85} \mathrm{Rb} /{ }^{87} \mathrm{Rb}=\mathbf{2 . 5 9 2 6 5}$ and atomic weight of $\mathrm{Rb}=85.46776$ (Catanzaro and others, 1969) were used. Strontium isotope-ratios were normalized to the Nier $(1938){ }^{86} \mathrm{Sr} /{ }^{88} \mathrm{Sr}$ value of 0.1194 . Measurements of ${ }^{87} \mathrm{Sr} /{ }^{86} \mathrm{Sr}$ in National Bureau of Standards $\mathrm{SrCO}_{3} 987$ during the period of analyses $(n=69)$ yield a weighted mean of $0.71024 \pm$ 0.00002 (standard error of the mean).

\section{RESULTS OF RUBIDIUM-STRONTIUM ANALYSES}

Locations of samples used in total-rock $\mathrm{Rb}-\mathrm{Sr}$ studies reported here are shown in figure 4. Locality numbers shown correspond to those in table 1 , which presents the analytical results. Samples represent all of the major geologic units shown in figure 2 with the exception of the quartz-porphyry and gabbro bodies. Rb-Sr ages obtained from these data (table 2) are discussed in the context of the generalized geologic units (fig. 2). In all cases these ages are calculated from whole-rock $\mathrm{Rb}-\mathrm{Sr}$ analyses. Those

${ }^{1}$ Following Brooks and others (1972), most authors, including us, have used (SUMS $/(n-2))^{1 / 2}$ as the equivalent of the mean square of weighted deviates (MSWD) of McIntyre and others (1966). As pointed out by Roddick (1978), however, the MSWD is actually equivalent to SUMS/ $(n-2)$ and not its square root. Values reported here as Index should be squared to be compared directly with MSWD and the cutoff value of 2.5 recommended for that quantity by Brooks and others (1972). 


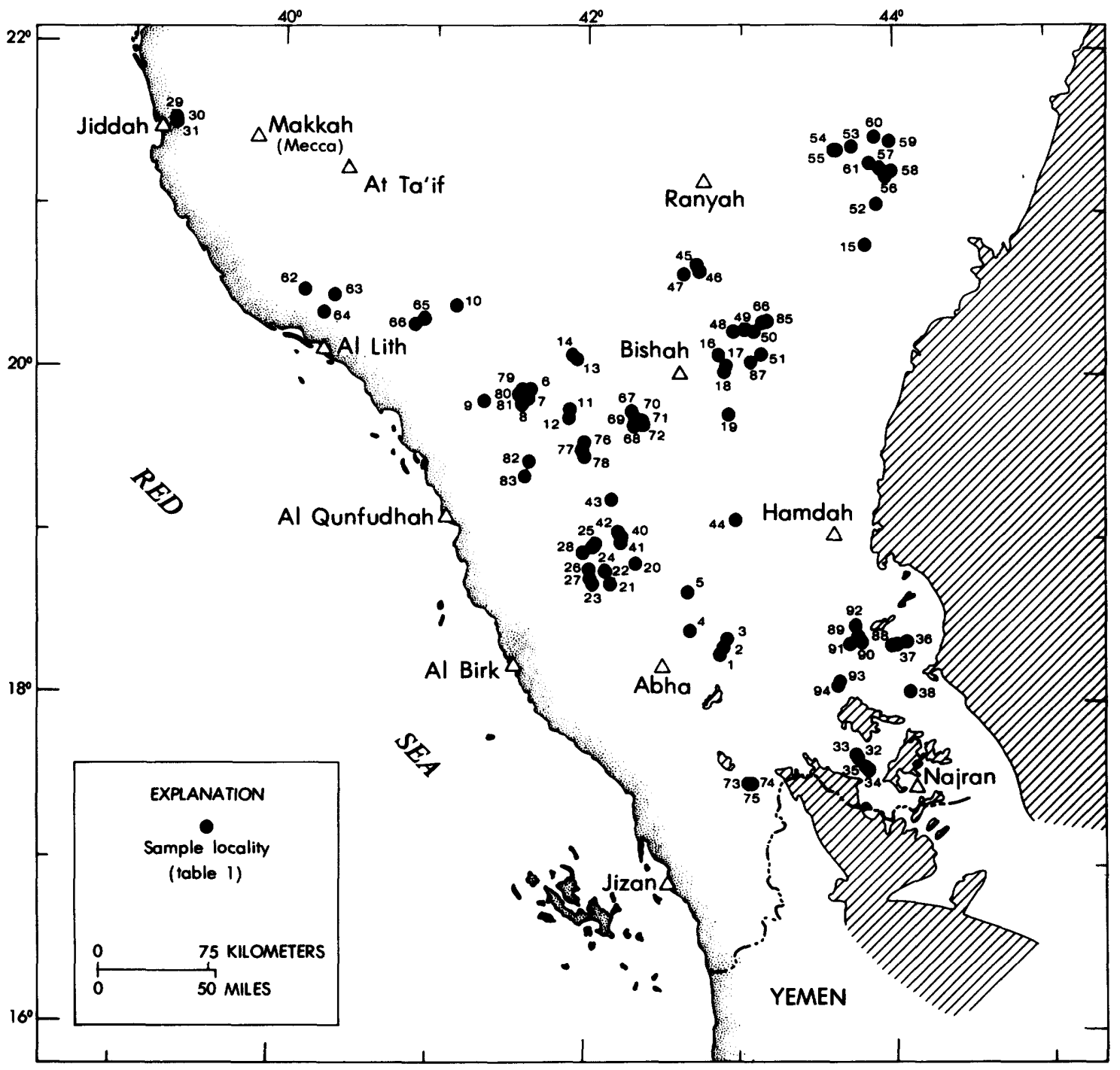

30 MINUTE QUADRANGLES

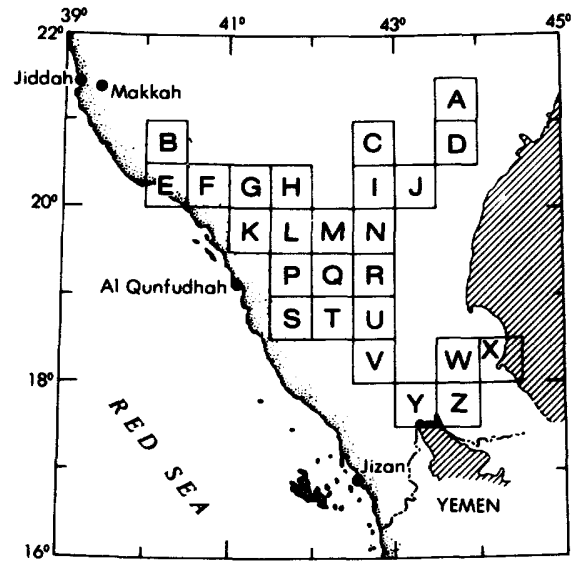
A. Bi'r Juqiug
B. Wadi Sadiyah
C. Wadi al Miyah
D. Jabal Yafikh
E. Al Lith
F. Jabal 'Afaf
G. Jabal Ibrahim
H. Al 'Aqiq
1. Junaynah
J. Al Qarah
K. Jabal Shada
L. Biljurshi
M. Wadi Tari

N. Wadi Harjab

P. Wadi Yiba

Q. An Nimas

R. Khadrah

S. Wadi Hali

T. Jabal 'Aya

U. Khaybar

V. Khamis Mushayt

W. Wadi Malahah

X. Wadi Wassat

Y. Wadi 'Aff

Z. Mayza

Figure 4.-Location of analyzed samples from the Arabian Shield (unpatterned areas) and of quadrangles. Cross-ruled areas represent areas of Paleozoic strata. Tertiary and Quaternary units are not shown. 
RESULTS OF RUBIDIUM-STRONTIUM ANALYSES

TABLE 1.-Analytical results for rocks of the Arabian Shield

\begin{tabular}{|c|c|c|c|c|c|c|c|c|}
\hline $\begin{array}{l}\text { Locality } \\
\text { No. }\end{array}$ & le No. & $\begin{array}{c}\text { North } \\
\text { lat }\end{array}$ & $\begin{array}{l}\text { East } \\
\text { long }\end{array}$ & $\begin{array}{c}\mathrm{Rb} \\
(\mathrm{ppm})\end{array}$ & $\begin{array}{c}\mathrm{Sr} \\
(\mathrm{ppm})\end{array}$ & ${ }^{87} \mathbf{R b} /{ }^{86} \mathrm{Sr}$ & ${ }^{87} \mathrm{Sr} /{ }^{86} \mathrm{Sr}$ & Rock type \\
\hline $\begin{array}{l}1--71-8-9 \mathrm{~A} \\
2--71-8-9 \mathrm{~B} \\
3--71-8-9 \mathrm{C}\end{array}$ & --- & $\begin{array}{l}18^{\circ} 18.6^{\prime} \\
18^{\circ} 20.5^{\prime} \\
18^{\circ} 23.5^{\prime}\end{array}$ & $\begin{array}{l}42^{\circ} 52.1^{\prime} \\
42^{\circ} 53.4^{\prime} \\
42^{\circ} 55.3^{\prime}\end{array}$ & $\begin{array}{l}345.7 \\
150.9 \\
156.1\end{array}$ & $\begin{array}{l}253.5 \\
349.9 \\
283.6\end{array}$ & $\begin{array}{l}3.958 \\
1.249 \\
1.594\end{array}$ & $\begin{array}{r}0.73905 \\
.71487 \\
.71788\end{array}$ & $\begin{array}{l}\text { Granodiorite-quartz monzonite } \\
\text { Do. } \\
\text { Do. }\end{array}$ \\
\hline $\begin{array}{r}71-8-9 D \\
71-8-9 E \\
71-8-9 F\end{array}$ & 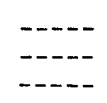 & $\begin{array}{l}18^{\circ} 26.7^{\prime} \\
18^{\circ} 26.7^{\prime} \\
18^{\circ} 26.7^{\prime}\end{array}$ & $\begin{array}{l}42^{\circ} 40.1^{\prime} \\
42^{\circ} 40.1^{\prime} \\
42^{\circ} 40.1^{\prime}\end{array}$ & $\begin{array}{l}107.6 \\
112.8 \\
113.7\end{array}$ & $\begin{array}{c}163.1 \\
71.17 \\
50.05\end{array}$ & $\begin{array}{l}1.911 \\
4.603 \\
6.608\end{array}$ & $\begin{array}{l}.72153 \\
.74846 \\
.76607\end{array}$ & $\begin{array}{l}\text { Garnet-bearing granite } \\
\text { Do. } \\
\text { Do. }\end{array}$ \\
\hline $5 \ldots-71-8-11 \mathrm{~K}$ & $K \ldots$ & $18^{\circ} 40.7^{\prime}$ & $42^{\circ} 39.5^{\prime}$ & 294.6 & 235.4 & 3.631 & .73467 & Quartz monzonite \\
\hline $\begin{array}{l}\text { 6-- -71-8-13H } \\
7---71-8-13 \mathrm{I} \\
8---71-8-13 \mathrm{~J}\end{array}$ & H.-- & $\begin{array}{l}19^{\circ} 55.0^{\prime} \\
19^{\circ} 51.0^{\prime} \\
19^{\circ} 50.0^{\prime}\end{array}$ & $\begin{array}{l}41^{\circ} 37.0^{\prime} \\
41^{\circ} 36.0^{\prime} \\
41^{\circ} 34.5^{\prime}\end{array}$ & $\begin{array}{l}39.9 \\
17.6 \\
58.5\end{array}$ & $\begin{array}{l}377 \\
358 \\
345\end{array}$ & $\begin{array}{l}.3058 \\
.1417 \\
.4838\end{array}$ & $\begin{array}{l}.70652 \\
.70442 \\
.70877\end{array}$ & $\begin{array}{l}\text { Quartz diorite } \\
\text { Do. } \\
\text { Do. }\end{array}$ \\
\hline $\begin{array}{r}9---71-8-14 \mathrm{~A} \\
10---71-8-14 \mathrm{~B}\end{array}$ & $\begin{array}{l}A \\
B \\
B---\end{array}$ & $\begin{array}{l}19^{\circ} 50.5^{\prime} \\
20^{\circ} 25.5^{\prime}\end{array}$ & $\begin{array}{l}41^{\circ} 19.0^{\prime} \\
41^{\circ} 08.5^{\prime}\end{array}$ & $\begin{array}{l}163.0 \\
131.9\end{array}$ & $\begin{array}{l}85.70 \\
222.4\end{array}$ & $\begin{array}{l}5.528 \\
1.718\end{array}$ & $\begin{array}{l}.75365 \\
.71930\end{array}$ & $\begin{array}{l}\text { Biotite, muscovite granite } \\
\text { Do. }\end{array}$ \\
\hline $\begin{array}{l}11---71-8-15 \mathrm{~A} \\
12---71-8-15 \mathrm{C} \\
13---71-8-15 \mathrm{D} \\
14---71-8-15 \mathrm{E}\end{array}$ & $\begin{array}{l}--- \\
-\cdots \\
---\end{array}$ & $\begin{array}{l}19^{\circ} 48.0^{\prime} \\
19^{\circ} 44.5^{\prime} \\
20^{\circ} 07.0^{\prime} \\
20^{\circ} 07.5^{\prime}\end{array}$ & $\begin{array}{l}41^{\circ} 52.5^{\prime} \\
41^{\circ} 52.5^{\prime} \\
41^{\circ} 55.0^{\prime} \\
41^{\circ} 54.0^{\prime}\end{array}$ & $\begin{array}{r}123.2 \\
24.68 \\
31.71 \\
49.22\end{array}$ & $\begin{array}{c}209.3 \\
493.5 \\
218.3 \\
52.94\end{array}$ & $\begin{array}{l}1.705 \\
.1447 \\
.4131 \\
2.6955\end{array}$ & $\begin{array}{l}.71888 \\
.70485 \\
.70709 \\
.72859\end{array}$ & $\begin{array}{l}\text { Quartz monzonite } \\
\text { Do. } \\
\text { Perthitic quartz monzonite } \\
\text { Do. }\end{array}$ \\
\hline $\begin{array}{r}15--71-8-18 \mathrm{M} \\
71-8-18 \mathrm{~N} \\
71-8-18 \mathrm{P}\end{array}$ & $\begin{array}{l}\text { M } \\
\text { N } \\
0 \\
-\cdots--\end{array}$ & $\begin{array}{l}20^{\circ} 48.6^{\prime} \\
20^{\circ} 48.6^{\prime} \\
20^{\circ} 48.6^{\prime}\end{array}$ & $\begin{array}{l}43^{\circ} 48.9^{\prime} \\
43^{\circ} 48.9^{\prime} \\
43^{\circ} 48.9^{\prime}\end{array}$ & $\begin{array}{l}57.00 \\
60.95 \\
52.05\end{array}$ & $\begin{array}{c}121.0 \\
51.02 \\
75.19\end{array}$ & $\begin{array}{l}1.364 \\
3.466 \\
2.006\end{array}$ & $\begin{array}{l}.71711 \\
.73383 \\
.72244\end{array}$ & $\begin{array}{c}\text { Rhyolite tuff } \\
\text { Do. } \\
\text { Do. }\end{array}$ \\
\hline $\begin{array}{r}16 \ldots-724-28 \mathrm{~J} \\
724-28 \mathrm{~K} \\
724-28 \mathrm{~L}\end{array}$ & & $\begin{array}{l}20^{\circ} 08.2^{\prime} \\
20^{\circ} 08.2^{\prime} \\
20^{\circ} 08.2^{\prime}\end{array}$ & $\begin{array}{l}42^{\circ} 51.0^{\prime} \\
42^{\circ} 51.0^{\prime} \\
42^{\circ} 51.0^{\prime}\end{array}$ & $\begin{array}{r}20.8 \\
23.1 \\
3.7\end{array}$ & $\begin{array}{l}245 \\
187 \\
208\end{array}$ & $\begin{array}{l}.2450 \\
.3584 \\
.0518\end{array}$ & $\begin{array}{l}.70528 \\
.70656 \\
.70312\end{array}$ & $\begin{array}{c}\text { Andesite tuff } \\
\text { Do. } \\
\text { Do. }\end{array}$ \\
\hline $\begin{array}{r}17--724-28 \mathrm{~N} \\
18--724-28 \mathrm{P} \\
19---724-29 \mathrm{H} \\
724-29 \mathrm{I}\end{array}$ & $\begin{array}{l}-\cdots \\
-\cdots- \\
-\cdots- \\
-\cdots-\end{array}$ & $\begin{array}{l}20^{\circ} 03.6^{\prime} \\
20^{\circ} 02.7^{\prime} \\
19^{\circ} 46.5^{\prime} \\
19^{\circ} 46.5^{\prime}\end{array}$ & $\begin{array}{l}42^{\circ} 54.3^{\prime} \\
42^{\circ} 54.2^{\prime} \\
42^{\circ} 55.4^{\prime} \\
42^{\circ} 55.4^{\prime}\end{array}$ & $\begin{array}{l}54.2 \\
41.5 \\
60.2 \\
92.1\end{array}$ & $\begin{array}{l}692 \\
680 \\
534 \\
545\end{array}$ & $\begin{array}{l}.2266 \\
.1766 \\
.3262 \\
.4887\end{array}$ & $\begin{array}{l}.70486 \\
.70439 \\
.70587 \\
.70760\end{array}$ & $\begin{array}{l}\text { Quartz diorite } \\
\text { Do. } \\
\text { Do. } \\
\text { Do. }\end{array}$ \\
\hline $\begin{array}{r}20 \\
21--725-6 \mathrm{~L} \\
--725-6 \mathrm{M} \\
725-6 \mathrm{~N} \\
22- \\
--725-6 \mathrm{P}\end{array}$ & 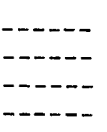 & $\begin{array}{l}18^{\circ} 52.0^{\prime} \\
18^{\circ} 43.7^{\prime} \\
18^{\circ} 43.7^{\prime} \\
18^{\circ} 48.2^{\prime}\end{array}$ & $\begin{array}{l}42^{\circ} 19.0^{\prime} \\
42^{\circ} 09.1^{\prime} \\
42^{\circ} 09.1^{\prime} \\
42^{\circ} 07.1^{\prime}\end{array}$ & $\begin{array}{l}202 \\
150 \\
160 \\
131\end{array}$ & $\begin{array}{c}101 \\
116 \\
28.4 \\
396\end{array}$ & $\begin{array}{c}5.830 \\
3.745 \\
16.50 \\
.9573\end{array}$ & $\begin{array}{l}.75419 \\
.73651 \\
.84947 \\
.71188\end{array}$ & $\begin{array}{l}\text { Quartz monzonite } \\
\text { Do. } \\
\text { Granite } \\
\text { Granodiorite }\end{array}$ \\
\hline 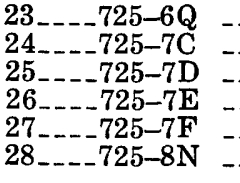 & 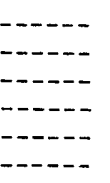 & $\begin{array}{l}18^{\circ} 44.0^{\prime} \\
18^{\circ} 57.9^{\prime} \\
18^{\circ} 58.5^{\prime} \\
18^{\circ} 49.0^{\prime} \\
18^{\circ} 45.6^{\prime} \\
18^{\circ} 56.0^{\prime}\end{array}$ & $\begin{array}{l}42^{\circ} 02.0^{\prime} \\
42^{\circ} 02.7^{\prime} \\
42^{\circ} 03.4^{\prime} \\
42^{\circ} 01.2^{\prime} \\
42^{\circ} 01.6^{\prime} \\
41^{\circ} 59.0^{\prime}\end{array}$ & $\begin{array}{l}56.1 \\
34.1 \\
33.2 \\
53.7 \\
21.6 \\
35.0\end{array}$ & $\begin{array}{l}388 \\
167 \\
370 \\
385 \\
366 \\
415\end{array}$ & $\begin{array}{l}.4178 \\
.5903 \\
.2600 \\
.4036 \\
.1723 \\
.2438\end{array}$ & $\begin{array}{l}.70773 \\
.70960 \\
.70600 \\
.70783 \\
.70548 \\
.70583\end{array}$ & $\begin{array}{c}\text { Granodiorite gneiss } \\
\text { Do. } \\
\text { Do. } \\
\text { Do. } \\
\text { Do. } \\
\text { Do. }\end{array}$ \\
\hline $\begin{array}{r}29+-742-15 \mathrm{~A} \\
742-15 \mathrm{~B} \\
30 \_-742-15 \mathrm{C} \\
31 \_-742-15 \mathrm{D}\end{array}$ & $\begin{array}{l}-\cdots- \\
-\cdots- \\
-\cdots- \\
----\end{array}$ & $\begin{array}{l}21^{\circ} 31.3^{\prime} \\
21^{\circ} 31.3^{\prime} \\
21^{\circ} 31.1^{\prime} \\
21^{\circ} 30.7^{\prime}\end{array}$ & $\begin{array}{l}39^{\circ} 16.0^{\prime} \\
39^{\circ} 16.0^{\prime} \\
39^{\circ} 16.0^{\prime} \\
39^{\circ} 16.3^{\prime}\end{array}$ & $\begin{array}{l}66.6 \\
62.3 \\
68.6 \\
66.9\end{array}$ & $\begin{array}{l}214 \\
239 \\
226 \\
214\end{array}$ & $\begin{array}{l}.9017 \\
.7532 \\
.8802 \\
.9035\end{array}$ & $\begin{array}{l}.71251 \\
.71081 \\
.71219 \\
.71237\end{array}$ & $\begin{array}{l}\text { Do. } \\
\text { Do. } \\
\text { Do. } \\
\text { Do. }\end{array}$ \\
\hline $\begin{array}{c}32-742-19 \mathrm{R} \\
742-19 \mathrm{~S} \\
742-19 \mathrm{~T} \\
33--742-19 \mathrm{U} \\
742-19 \mathrm{~V} \\
742-19 \mathrm{~W}\end{array}$ & $\begin{array}{l}-\cdots- \\
-\cdots- \\
-\cdots- \\
-\cdots \\
-\cdots-\end{array}$ & $\begin{array}{l}17^{\circ} 40.8^{\prime} \\
17^{\circ} 40.8^{\prime} \\
17^{\circ} 40.8^{\prime} \\
17^{\circ} 41.2^{\prime} \\
17^{\circ} 41.2^{\prime} \\
17^{\circ} 41.2^{\prime}\end{array}$ & $\begin{array}{l}43^{\circ} 45.1^{\prime} \\
43^{\circ} 45.1^{\prime} \\
43^{\circ} 45.1^{\prime} \\
43^{\circ} 45.0^{\prime} \\
43^{\circ} 45.0^{\prime} \\
43^{\circ} 45.0^{\prime}\end{array}$ & $\begin{array}{l}12.5 \\
12.2 \\
22.1 \\
26.7 \\
22.9 \\
28.7\end{array}$ & $\begin{array}{l}114 \\
136 \\
150 \\
212 \\
180 \\
138\end{array}$ & $\begin{array}{l}.3169 \\
.2609 \\
.4282 \\
.3648 \\
.3683 \\
.6013\end{array}$ & $\begin{array}{l}.70615 \\
.70618 \\
.70761 \\
.70710 \\
.70717 \\
.70908\end{array}$ & $\begin{array}{c}\text { Metadacite } \\
\text { Do. } \\
\text { Do. } \\
\text { Do. } \\
\text { Do. } \\
\text { Do. }\end{array}$ \\
\hline $\begin{array}{r}34-742-20 \mathrm{~A} \\
742-20 \mathrm{~B} \\
35--742-20 \mathrm{C} \\
742-20 \mathrm{D} \\
742-20 \mathrm{E} \\
742-20 \mathrm{~F} \\
742-20 \mathrm{G}\end{array}$ & 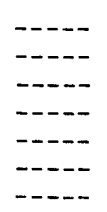 & $\begin{array}{l}17^{\circ} 36.2^{\prime} \\
17^{\circ} 36.2^{\prime} \\
17^{\circ} 36.3^{\prime} \\
17^{\circ} 36.3^{\prime} \\
17^{\circ} 36.3^{\prime} \\
17^{\circ} 36.3^{\prime} \\
17^{\circ} 36.3^{\prime}\end{array}$ & $\begin{array}{l}43^{\circ} 49.1^{\prime} \\
43^{\circ} 49.1^{\prime} \\
43^{\circ} 49.0^{\prime} \\
43^{\circ} 49.0^{\prime} \\
43^{\circ} 49.0^{\prime} \\
43^{\circ} 49.0^{\prime} \\
43^{\circ} 49.0^{\prime}\end{array}$ & $\begin{array}{l}38.6 \\
94.2 \\
51.9 \\
40.7 \\
45.6 \\
55.9 \\
94.6\end{array}$ & $\begin{array}{l}570 \\
157 \\
304 \\
602 \\
396 \\
379 \\
207\end{array}$ & $\begin{array}{l}.1958 \\
1.736 \\
.4936 \\
.1955 \\
.3332 \\
.4261 \\
1.324\end{array}$ & $\begin{array}{l}.70489 \\
.71905 \\
.70719 \\
.70472 \\
.70586 \\
.70678 \\
.71493\end{array}$ & $\begin{array}{l}\text { Quartz diorite } \\
\text { Granodiorite } \\
\text { Do. } \\
\text { Quartz diorite } \\
\text { Granodiorite } \\
\text { Do. } \\
\text { Do. }\end{array}$ \\
\hline $\begin{array}{r}36-742-20 \mathrm{I} \\
742-20 \mathrm{~J} \\
742-20 \mathrm{~K} \\
742-20 \mathrm{~L}\end{array}$ & $\begin{array}{l}-1 \\
-\cdots-1 \\
-\cdots-1\end{array}$ & $\begin{array}{l}18^{\circ} 23^{\prime} \\
18^{\circ} 23^{\prime} \\
18^{\circ} 23^{\prime} \\
18^{\circ} 23^{\prime}\end{array}$ & $\begin{array}{l}44^{\circ} 04^{\prime} \\
44^{\circ} 04^{\prime} \\
44^{\circ} 04^{\prime} \\
44^{\circ} 04^{\prime}\end{array}$ & $\begin{array}{l}25.5 \\
35.9 \\
26.2 \\
16.4\end{array}$ & $\begin{array}{l}526 \\
562 \\
704 \\
626\end{array}$ & $\begin{array}{l}.1403 \\
.1844 \\
.1075 \\
.0760\end{array}$ & $\begin{array}{l}.70427 \\
.70467 \\
.70394 \\
.70378\end{array}$ & $\begin{array}{c}\text { Quartz diorite } \\
\text { Do. } \\
\text { Do. } \\
\text { Do. }\end{array}$ \\
\hline $\begin{array}{r}37-742-21 \mathrm{~F} \\
742-21 \mathrm{G} \\
742-21 \mathrm{H}\end{array}$ & $\begin{array}{l}-\cdots- \\
-\cdots- \\
-\cdots-\end{array}$ & $\begin{array}{l}18^{\circ} 22^{\prime} \\
18^{\circ} 22^{\prime} \\
18^{\circ} 22^{\prime}\end{array}$ & $\begin{array}{l}44^{\circ} 00^{\prime} \\
44^{\circ} 00^{\prime} \\
44^{\circ} 00^{\prime}\end{array}$ & $\begin{array}{l}44.1 \\
31.5 \\
37.2\end{array}$ & $\begin{array}{l}530 \\
615 \\
767\end{array}$ & $\begin{array}{l}.2405 \\
.1482 \\
.1401\end{array}$ & $\begin{array}{l}.70521 \\
.70412 \\
.70402\end{array}$ & $\begin{array}{l}\text { Do. } \\
\text { Do. } \\
\text { Do. }\end{array}$ \\
\hline $\begin{array}{r}38-742-21 \mathrm{~A} \\
742-21 \mathrm{~B}\end{array}$ & $-\cdots$ & $\begin{array}{l}18^{\circ} 05^{\prime} \\
18^{\circ} 05^{\prime}\end{array}$ & $\begin{array}{l}44^{\circ} 05^{\prime} \\
44^{\circ} 05^{\prime}\end{array}$ & $\begin{array}{l}29.5 \\
18.4\end{array}$ & $\begin{array}{l}496 \\
537\end{array}$ & $\begin{array}{l}.1715 \\
.0989\end{array}$ & $\begin{array}{l}.70455 \\
.70387\end{array}$ & $\begin{array}{l}\text { Do. } \\
\text { Do. }\end{array}$ \\
\hline $\begin{array}{r}40--742-22 \mathrm{~A} \\
742-22 \mathrm{~B} \\
742-22 \mathrm{C} \\
742-22 \mathrm{D} \\
742-22 \mathrm{E} \\
41-\ldots-742-22 \mathrm{~F}\end{array}$ & $\begin{array}{l}---- \\
-\cdots- \\
-\cdots- \\
-\cdots- \\
-\cdots-\end{array}$ & $\begin{array}{l}19^{\circ} 01^{\prime} \\
19^{\circ} 01^{\prime} \\
19^{\circ} 01^{\prime} \\
19^{\circ} 01^{\prime} \\
19^{\circ} 01^{\prime} \\
19^{\circ} 00^{\prime}\end{array}$ & $\begin{array}{l}42^{\circ} 13^{\prime} \\
42^{\circ} 13^{\prime} \\
42^{\circ} 13^{\prime} \\
42^{\circ} 13^{\prime} \\
42^{\circ} 13^{\prime} \\
42^{\circ} 13^{\prime}\end{array}$ & $\begin{array}{l}60.2 \\
59.5 \\
63.6 \\
57.3 \\
69.8 \\
64.4\end{array}$ & $\begin{array}{r}62.5 \\
62.9 \\
96.8 \\
61.9 \\
92.0 \\
132\end{array}$ & $\begin{array}{l}2.797 \\
2.746 \\
1.902 \\
2.686 \\
2.200 \\
1.410\end{array}$ & $\begin{array}{l}.73433 \\
.73318 \\
.72477 \\
.73335 \\
.72724 \\
.71638\end{array}$ & $\begin{array}{c}\text { Granodiorite gneiss } \\
\text { Do. } \\
\text { Do. } \\
\text { Do. } \\
\text { Do. } \\
\text { Do. }\end{array}$ \\
\hline
\end{tabular}


TABLE 1.-Analytical results for rocks of the Arabian Shield-Continued

\begin{tabular}{|c|c|c|c|c|c|c|c|c|}
\hline $\begin{array}{c}\text { Locality } \\
\text { No. }\end{array}$ & No. & $\begin{array}{c}\text { North } \\
\text { lat }\end{array}$ & $\begin{array}{l}\text { East } \\
\text { long }\end{array}$ & $\underset{(\mathrm{ppm})}{\mathrm{Rb}}$ & $\underset{(\mathrm{ppm})}{\mathrm{Sr}}$ & ${ }^{8 \pi} \mathrm{Rb} /{ }^{86} \mathrm{Sr}$ & ${ }^{87} \mathrm{Sr} /{ }^{80} \mathrm{Sr}$ & Rock type \\
\hline $\begin{array}{r}42-742-23 \mathrm{~F} \\
742-23 \mathrm{G} \\
742-23 \mathrm{H} \\
742-23 \mathrm{I}\end{array}$ & $\begin{array}{l}---1 \\
----1 \\
---1 \\
---1\end{array}$ & $\begin{array}{l}19^{\circ} 03.0^{\prime} \\
19^{\circ} 03.0^{\prime} \\
19^{\circ} 03.0^{\prime} \\
19^{\circ} 07^{\prime}\end{array}$ & $\begin{array}{l}42^{\circ} 12.0^{\prime} \\
42^{\circ} 12.0^{\prime} \\
42^{\circ} 12.0^{\prime} \\
42^{\circ} 09^{\prime}\end{array}$ & $\begin{array}{r}46.6 \\
36.2 \\
1.1 \\
35.4\end{array}$ & $\begin{array}{l}329 \\
422 \\
488 \\
365\end{array}$ & $\begin{array}{r}0.4097 \\
.2479 \\
.0063 \\
.2807\end{array}$ & $\begin{array}{r}0.70765 \\
.70559 \\
.70282 \\
.70616\end{array}$ & $\begin{array}{c}\text { Quartz diorite } \\
\text { Do. } \\
\text { Do. } \\
\text { Do. }\end{array}$ \\
\hline $\begin{array}{c}43 \\
742-24 \mathrm{C} \\
742-24 \mathrm{D} \\
742-24 \mathrm{E} \\
742-24 \mathrm{~F} \\
742-24 \mathrm{G} \\
742-24 \mathrm{H} \\
742-24 \mathrm{I}\end{array}$ & 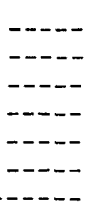 & $\begin{array}{l}19^{\circ} 15^{\prime} \\
19^{\circ} 15^{\prime} \\
19^{\circ} 15^{\prime} \\
19^{\circ} 15^{\prime} \\
19^{\circ} 15^{\prime} \\
19^{\circ} 15^{\prime} \\
19^{\circ} 15^{\prime}\end{array}$ & $\begin{array}{l}42^{\circ} 09^{\prime} \\
42^{\circ} 09^{\prime} \\
42^{\circ} 09^{\prime} \\
42^{\circ} 09^{\prime} \\
42^{\circ} 09^{\prime} \\
42^{\circ} 09^{\prime} \\
42^{\circ} 09^{\prime}\end{array}$ & $\begin{array}{r}54.8 \\
14.0 \\
34.1 \\
36.8 \\
12.9 \\
8.5 \\
22.2\end{array}$ & $\begin{array}{l}170 \\
228 \\
209 \\
345 \\
662 \\
409 \\
255\end{array}$ & $\begin{array}{l}.9352 \\
.1779 \\
.4721 \\
.3085 \\
.0565 \\
.0601 \\
.2521\end{array}$ & $\begin{array}{l}.71142 \\
.70477 \\
.70739 \\
.70662 \\
.70320 \\
.70358 \\
.70575\end{array}$ & $\begin{array}{l}\text { Meta-andesite or dacite } \\
\text { Do. } \\
\text { Do. } \\
\text { Do. } \\
\text { Do. } \\
\text { Do. } \\
\text { Do. }\end{array}$ \\
\hline $\begin{aligned} & 44- 742-24 \mathrm{~J} \\
& 742-24 \mathrm{~K} \\
& 742-24 \mathrm{~L} \\
& 742-24 \mathrm{M}\end{aligned}$ & $\begin{array}{l}---1 \\
-\cdots- \\
---1\end{array}$ & $\begin{array}{l}19^{\circ} 08^{\prime} \\
19^{\circ} 08^{\prime} \\
19^{\circ} 08^{\prime} \\
19^{\circ} 08^{\prime}\end{array}$ & $\begin{array}{l}42^{\circ} 58^{\prime} \\
42^{\circ} 58^{\prime} \\
42^{\circ} 58^{\prime} \\
42^{\circ} 58^{\prime}\end{array}$ & $\begin{array}{l}21.4 \\
50.5 \\
50.5 \\
22.0\end{array}$ & $\begin{array}{c}167 \\
58.7 \\
52.0 \\
109\end{array}$ & $\begin{array}{l}.3704 \\
2.494 \\
2.818 \\
.5837\end{array}$ & $\begin{array}{l}.70623 \\
.72925 \\
.73438 \\
.70839\end{array}$ & $\begin{array}{c}\text { Meta-andesite } \\
\text { Do. } \\
\text { Do. } \\
\text { Do. }\end{array}$ \\
\hline $\begin{array}{c}45--742-25 \mathrm{~J} \\
46--742-25 \mathrm{~K} \\
-742-25 \mathrm{~L}\end{array}$ & $-\cdots$ & $\begin{array}{l}20^{\circ} 40.7^{\prime} \\
20^{\circ} 40.1^{\prime} \\
20^{\circ} 40.1^{\prime}\end{array}$ & $\begin{array}{l}42^{\circ} 42.9^{\prime} \\
42^{\circ} 43.2^{\prime} \\
42^{\circ} 43.2^{\prime}\end{array}$ & $\begin{array}{l}113 \\
118 \\
121\end{array}$ & $\begin{array}{l}142 \\
165 \\
141\end{array}$ & $\begin{array}{l}2.312 \\
2.066 \\
2.481\end{array}$ & $\begin{array}{l}.72418 \\
.72176 \\
.72492\end{array}$ & $\begin{array}{l}\text { Quartz monzonite } \\
\text { Do. } \\
\text { Do. }\end{array}$ \\
\hline $\begin{array}{c}47-742-25 \mathrm{M} \\
742-25 \mathrm{~N} \\
742-25 \mathrm{P} \\
742-25 \mathrm{Q} \\
742-25 \mathrm{R} \\
742-25 \mathrm{~S} \\
742-25 \mathrm{~T} \\
742-25 \mathrm{U}\end{array}$ & $\begin{array}{l}---- \\
---- \\
---- \\
---- \\
---- \\
--- \\
---- \\
----\end{array}$ & $\begin{array}{l}20^{\circ} 38.4^{\prime} \\
20^{\circ} 38.4^{\prime} \\
20^{\circ} 38.4^{\prime} \\
20^{\circ} 38.4^{\prime} \\
20^{\circ} 38.4^{\prime} \\
20^{\circ} 38.4^{\prime} \\
20^{\circ} 38.4^{\prime} \\
20^{\circ} 38.4^{\prime}\end{array}$ & $\begin{array}{l}42^{\circ} 37.2^{\prime} \\
42^{\circ} 37.2^{\prime} \\
42^{\circ} 37.2^{\prime} \\
42^{\circ} 37.2^{\prime} \\
42^{\circ} 37.2^{\prime} \\
42^{\circ} 37.2^{\prime} \\
42^{\circ} 37.2^{\prime} \\
42^{\circ} 37.2^{\prime}\end{array}$ & $\begin{array}{r}1.5 \\
2.3 \\
1.0 \\
6.2 \\
1.1 \\
20.8 \\
11.7 \\
8.8\end{array}$ & $\begin{array}{c}43.4 \\
53.5 \\
46.1 \\
41.0 \\
46.7 \\
270 \\
327 \\
232\end{array}$ & $\begin{array}{l}.1024 \\
.1222 \\
.0607 \\
.4347 \\
.0695 \\
.2224 \\
.1033 \\
.1090\end{array}$ & $\begin{array}{l}.70463 \\
.70497 \\
.70483 \\
.70806 \\
.70445 \\
.70508 \\
.70439 \\
.70435\end{array}$ & $\begin{array}{c}\text { Meta-andesite } \\
\text { Do. } \\
\text { Do. } \\
\text { Do. } \\
\text { Do. } \\
\text { Do. } \\
\text { Do. } \\
\text { Do. }\end{array}$ \\
\hline $\begin{array}{r}48 \ldots-742-26 \mathrm{~A} \\
742-26 \mathrm{~B} \\
49 \\
--742-26 \mathrm{C} \\
742-26 \mathrm{D} \\
742-26 \mathrm{E} \\
50 \_-742-26 \mathrm{~F} \\
742-26 \mathrm{G}\end{array}$ & 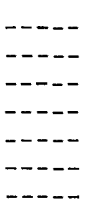 & $\begin{array}{l}20^{\circ} 16.7^{\prime} \\
20^{\circ} 16.7^{\prime} \\
20^{\circ} 17.9^{\prime} \\
20^{\circ} 17.9^{\prime} \\
20^{\circ} 17.9^{\prime} \\
20^{\circ} 17.2^{\prime} \\
20^{\circ} 17.2^{\prime}\end{array}$ & $\begin{array}{l}42^{\circ} 57.2^{\prime} \\
42^{\circ} 57.2^{\prime} \\
43^{\circ} 01.1^{\prime} \\
43^{\circ} 01.1^{\prime} \\
43^{\circ} 01.1^{\prime} \\
43^{\circ} 05.0^{\prime} \\
43^{\circ} 05.0^{\prime}\end{array}$ & $\begin{array}{c}69.7 \\
97.6 \\
115 \\
65.0 \\
137 \\
75.2 \\
107\end{array}$ & $\begin{array}{l}401 \\
299 \\
270 \\
311 \\
264 \\
340 \\
262\end{array}$ & $\begin{array}{l}.5030 \\
.9439 \\
1.235 \\
.6048 \\
1.505 \\
.6404 \\
1.182\end{array}$ & $\begin{array}{l}.70769 \\
.71159 \\
.71435 \\
.70866 \\
.71665 \\
.70903 \\
.71347\end{array}$ & $\begin{array}{c}\text { Granodiorite } \\
\text { Do. } \\
\text { Do. } \\
\text { Do. } \\
\text { Do. } \\
\text { Do. } \\
\text { Do. }\end{array}$ \\
\hline $\begin{array}{r}51-742-26 \mathrm{M} \\
742-26 \mathrm{~N} \\
742-26 \mathrm{P} \\
742-26 \mathrm{Q} \\
742-26 \mathrm{R}\end{array}$ & $\begin{array}{l}---1 \\
---- \\
-\cdots- \\
----\end{array}$ & $\begin{array}{l}20^{\circ} 08.9^{\prime} \\
20^{\circ} 08.9^{\prime} \\
20^{\circ} 08.9^{\prime} \\
20^{\circ} 08.9^{\prime} \\
20^{\circ} 08.9^{\prime}\end{array}$ & $\begin{array}{l}43^{\circ} 08.5^{\prime} \\
43^{\circ} 08.5^{\prime} \\
43^{\circ} 08.5^{\prime} \\
43^{\circ} 08.5^{\prime} \\
43^{\circ} 08.5^{\prime}\end{array}$ & $\begin{array}{l}25.4 \\
24.2 \\
82.2 \\
19.1 \\
74.8\end{array}$ & $\begin{array}{l}794 \\
886 \\
316 \\
764 \\
304\end{array}$ & $\begin{array}{l}.0925 \\
.0790 \\
.7537 \\
.0723 \\
.7119\end{array}$ & $\begin{array}{l}.70359 \\
.70380 \\
.70945 \\
.70347 \\
.70908\end{array}$ & $\begin{array}{l}\text { Quartz diorite } \\
\text { Do. } \\
\text { Aplite } \\
\text { Quartz diorite } \\
\text { Aplite }\end{array}$ \\
\hline $\begin{array}{r}52-742-27 \mathrm{D} \\
742-27 \mathrm{E} \\
742-27 \mathrm{~F}\end{array}$ & & $\begin{array}{l}21^{\circ} 04.0^{\prime} \\
21^{\circ} 04.0^{\prime} \\
21^{\circ} 04.0^{\prime}\end{array}$ & $\begin{array}{l}43^{\circ} 53.6^{\prime} \\
43^{\circ} 53.6^{\prime} \\
43^{\circ} 53.9^{\prime}\end{array}$ & $\begin{array}{l}81 \\
82 \\
92\end{array}$ & $\begin{array}{l}312 \\
352 \\
208\end{array}$ & $\begin{array}{r}.7500 \\
.6743 \\
1.2814\end{array}$ & $\begin{array}{l}.70997 \\
.70911 \\
.71572\end{array}$ & $\begin{array}{l}\text { Rhyolite ash-flow } \\
\text { Do. } \\
\text { Do. }\end{array}$ \\
\hline $\begin{aligned} 53- & 742-27 \mathrm{I} \\
& 742-27 \mathrm{~J} \\
& 742-27 \mathrm{~K}\end{aligned}$ & $\begin{array}{l}--- \\
-\cdots-\end{array}$ & $\begin{array}{l}21^{\circ} 25.2^{\prime} \\
21^{\circ} 25.2^{\prime} \\
21^{\circ} 25.2^{\prime}\end{array}$ & $\begin{array}{l}43^{\circ} 43.5^{\prime} \\
43^{\circ} 43.5^{\prime} \\
43^{\circ} 43.5^{\prime}\end{array}$ & $\begin{array}{l}14 \\
39 \\
40.8\end{array}$ & $\begin{array}{l}650 \\
390 \\
620\end{array}$ & $\begin{array}{l}.0648 \\
.2899 \\
.1904\end{array}$ & $\begin{array}{l}.70397 \\
.70596 \\
.70508\end{array}$ & $\begin{array}{c}\text { Pyroxene andesite } \\
\text { Do. } \\
\text { Basaltic andesite }\end{array}$ \\
\hline $\begin{array}{r}54--742-27 \mathrm{~L} \\
742-27 \mathrm{M}\end{array}$ & $=---$ & $\begin{array}{l}21^{\circ} 23.7^{\prime} \\
21^{\circ} 23.7^{\prime}\end{array}$ & $\begin{array}{l}43^{\circ} 37.9^{\prime} \\
43^{\circ} 37.9^{\prime}\end{array}$ & $\begin{array}{l}87.0 \\
90.6\end{array}$ & $\begin{array}{l}82.1 \\
95.2\end{array}$ & $\begin{array}{l}3.073 \\
2.758\end{array}$ & $\begin{array}{l}.73137 \\
.72862\end{array}$ & $\begin{array}{l}\text { Rhyolite ash-flow } \\
\text { Do. }\end{array}$ \\
\hline $\begin{array}{r}55+742-27 \mathrm{~N} \\
742-27 \mathrm{P} \\
742-27 \mathrm{Q} \\
742-27 \mathrm{R} \\
742-27 \mathrm{~S}\end{array}$ & $\begin{array}{l}-\cdots- \\
-\cdots- \\
-\cdots- \\
-\cdots-\end{array}$ & $\begin{array}{l}21^{\circ} 23.5^{\prime} \\
21^{\circ} 23.5^{\prime} \\
21^{\circ} 23.5^{\prime} \\
21^{\circ} 23.5^{\prime} \\
21^{\circ} 23.5^{\prime}\end{array}$ & $\begin{array}{l}43^{\circ} 37.6^{\prime} \\
43^{\circ} 37.6^{\prime} \\
43^{\circ} 37.6^{\prime} \\
43^{\circ} 37.6^{\prime} \\
43^{\circ} 37.6^{\prime}\end{array}$ & $\begin{array}{l}36.6 \\
13.0 \\
13.0 \\
32.2 \\
42.4\end{array}$ & $\begin{array}{l}698 \\
358 \\
358 \\
465 \\
619\end{array}$ & $\begin{array}{l}.1516 \\
.1050 \\
.1200 \\
.2000 \\
.1979\end{array}$ & $\begin{array}{l}.70449 \\
.70412 \\
.70414 \\
.70558 \\
.70556\end{array}$ & $\begin{array}{l}\text { Basaltic andesite } \\
\text { Volcanic breccia } \\
\text { Basaltic andesite } \\
\text { Volcanic breccia } \\
\text { Do. }\end{array}$ \\
\hline $\begin{array}{r}56-742-28 \mathrm{~A} \\
742-28 \mathrm{~B} \\
742-28 \mathrm{C} \\
57--742-28 \mathrm{D} \\
742-28 \mathrm{E}\end{array}$ & $\begin{array}{l}-\cdots- \\
-\cdots- \\
-\cdots- \\
-\cdots-\end{array}$ & $\begin{array}{l}21^{\circ} 14.2^{\prime} \\
21^{\circ} 14.2^{\prime} \\
21^{\circ} 14.2^{\prime} \\
21^{\circ} 16.8^{\prime} \\
21^{\circ} 16.8^{\prime}\end{array}$ & $\begin{array}{l}43^{\circ} 56.9^{\prime} \\
43^{\circ} 56.9^{\prime} \\
43^{\circ} 56.9^{\prime} \\
43^{\circ} 54.9^{\prime} \\
43^{\circ} 54.9^{\prime}\end{array}$ & $\begin{array}{c}90.4 \\
89.7 \\
93.7 \\
107 \\
94.2\end{array}$ & $\begin{array}{l}334 \\
338 \\
350 \\
296 \\
346\end{array}$ & $\begin{array}{c}.7823 \\
.7667 \\
.7752 \\
1.044 \\
.7865\end{array}$ & $\begin{array}{l}.71019 \\
.71024 \\
.71003 \\
.71222 \\
.71007\end{array}$ & $\begin{array}{c}\text { Granodiorite } \\
\text { Do. } \\
\text { Do. } \\
\text { Do. } \\
\text { Do. }\end{array}$ \\
\hline $\begin{array}{r}58 \ldots-742-28 \mathrm{~F} \\
742-28 \mathrm{G} \\
742-28 \mathrm{H}\end{array}$ & $\begin{array}{ll}--\cdots \\
---1-\end{array}$ & $\begin{array}{l}21^{\circ} 16.5^{\prime} \\
21^{\circ} 16.5^{\prime} \\
21^{\circ} 16.5^{\prime}\end{array}$ & $\begin{array}{l}43^{\circ} 59.4^{\prime} \\
43^{\circ} 59.4^{\prime} \\
43^{\circ} 59.4^{\prime}\end{array}$ & $\begin{array}{r}6.8 \\
15.7 \\
11.7\end{array}$ & $\begin{array}{l}773 \\
726 \\
662\end{array}$ & $\begin{array}{l}.0254 \\
.0625 \\
.0511\end{array}$ & $\begin{array}{l}.70352 \\
.70380 \\
.70370\end{array}$ & $\begin{array}{l}\text { Hornblende diorite } \\
\text { Do. } \\
\text { Do. }\end{array}$ \\
\hline $\begin{array}{r}59-742-28 \mathrm{I} \\
742-28 \mathrm{~J} \\
742-28 \mathrm{~K}\end{array}$ & 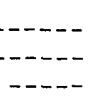 & $\begin{array}{l}21^{\circ} 28.1^{\prime} \\
21^{\circ} 28.1^{\prime} \\
21^{\circ} 28.1^{\prime}\end{array}$ & $\begin{array}{l}43^{\circ} 58.6^{\prime} \\
43^{\circ} 58.6^{\prime} \\
43^{\circ} 58.6^{\prime}\end{array}$ & $\begin{array}{l}147 \\
157 \\
148\end{array}$ & $\begin{array}{l}312 \\
332 \\
306\end{array}$ & $\begin{array}{l}1.364 \\
1.371 \\
1.400\end{array}$ & $\begin{array}{l}.71619 \\
.71553 \\
.71540\end{array}$ & $\begin{array}{l}\text { Quartz monzonite } \\
\text { Do. } \\
\text { Do. }\end{array}$ \\
\hline $\begin{array}{r}60-742-28 \mathrm{~L} \\
742-28 \mathrm{M}\end{array}$ & ---- & $\begin{array}{l}21^{\circ} 29.6^{\prime} \\
21^{\circ} 29.6^{\prime}\end{array}$ & $\begin{array}{l}43^{\circ} 52.5^{\prime} \\
43^{\circ} 52.5^{\prime}\end{array}$ & $\begin{array}{l}110 \\
112\end{array}$ & $\begin{array}{l}338 \\
322\end{array}$ & $\begin{array}{l}.9427 \\
1.045\end{array}$ & $\begin{array}{l}.71157 \\
.71213\end{array}$ & $\begin{array}{c}\text { Granodiorite } \\
\text { Do. }\end{array}$ \\
\hline $\begin{array}{r}61--742-28 \mathrm{~N} \\
742-28 \mathrm{P}\end{array}$ & $-\cdots$ & $\begin{array}{l}21^{\circ} 19.5^{\prime} \\
21^{\circ} 19.5^{\prime}\end{array}$ & $\begin{array}{l}43^{\circ} 50.6^{\prime} \\
43^{\circ} 50.6^{\prime}\end{array}$ & $\begin{array}{l}108 \\
110\end{array}$ & $\begin{array}{l}195 \\
177\end{array}$ & $\begin{array}{l}1.603 \\
1.808\end{array}$ & $\begin{array}{l}.71764 \\
.71949\end{array}$ & $\begin{array}{l}\text { Quartz monzonite } \\
\text { Do. }\end{array}$ \\
\hline
\end{tabular}


TABLE 1.-Analytical results for rocks of the Arabian Shield-Continued

\begin{tabular}{|c|c|c|c|c|c|c|c|}
\hline $\begin{array}{c}\text { Locality } \\
\text { No. }\end{array}$ & $\begin{array}{c}\text { North } \\
\text { lat }\end{array}$ & $\begin{array}{l}\text { East } \\
\text { long }\end{array}$ & $\begin{array}{c}\mathbf{R b} \\
(\mathrm{ppm})\end{array}$ & $\begin{array}{c}\mathrm{Sr} \\
(\mathrm{ppm})\end{array}$ & ${ }^{87} \mathbf{R b} /{ }^{86} \mathrm{Sr}$ & ${ }^{8 \pi} \mathrm{Sr} /{ }^{80} \mathrm{Sr}$ & Rock type \\
\hline 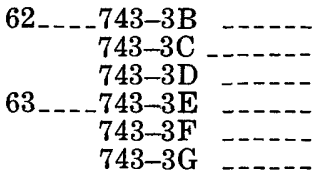 & $\begin{array}{l}20^{\circ} 30.9^{\prime} \\
20^{\circ} 30.9^{\prime} \\
20^{\circ} 30.9^{\prime} \\
20^{\circ} 29.5^{\prime} \\
20^{\circ} 29.5^{\prime} \\
20^{\circ} 29.5^{\prime}\end{array}$ & $\begin{array}{l}40^{\circ} 08.5^{\prime} \\
40^{\circ} 08.5^{\prime} \\
40^{\circ} 08.5^{\prime} \\
40^{\circ} 20.6^{\prime} \\
40^{\circ} 20.6^{\prime} \\
40^{\circ} 20.6^{\prime}\end{array}$ & $\begin{array}{l}28.6 \\
26.4 \\
18.4 \\
16.3 \\
10.1 \\
13.7\end{array}$ & $\begin{array}{l}500 \\
512 \\
609 \\
578 \\
492 \\
558\end{array}$ & $\begin{array}{l}0.1656 \\
.1490 \\
.0872 \\
.0818 \\
.0593 \\
.0708\end{array}$ & $\begin{array}{l}0.70469 \\
.70448 \\
.70344 \\
.70384 \\
.70337 \\
.70346\end{array}$ & $\begin{array}{c}\text { Quartz diorite } \\
\text { Do. } \\
\text { Do. } \\
\text { Do. } \\
\text { Do. } \\
\text { Do. }\end{array}$ \\
\hline $\begin{array}{r}64 \ldots \\
743-3 \mathrm{I}=\ldots \\
743-3 J \\
-\ldots\end{array}$ & $\begin{array}{l}20^{\circ} 22.6^{\prime} \\
20^{\circ} 22.6^{\prime} \\
20^{\circ} 22.6^{\prime}\end{array}$ & $\begin{array}{l}40^{\circ} 15.9^{\prime} \\
40^{\circ} 15.9^{\prime} \\
40^{\circ} 15.9^{\prime}\end{array}$ & $\begin{array}{c}4.82 \\
0.42 \\
12.9\end{array}$ & $\begin{array}{l}548 \\
416 \\
347\end{array}$ & $\begin{array}{l}.0255 \\
.0030 \\
.1077\end{array}$ & $\begin{array}{l}.70335 \\
.70292 \\
.70468\end{array}$ & $\begin{array}{c}\text { Metabasalt } \\
\text { Do. } \\
\text { Do. }\end{array}$ \\
\hline $\begin{array}{r}65 \ldots \\
743-4 \mathrm{G} \\
743-4 \mathrm{H}=\ldots \\
743-4 \mathrm{~J} \\
\mathbf{7}\end{array}$ & $\begin{array}{l}20^{\circ} 20.6^{\prime} \\
20^{\circ} 20.6^{\prime} \\
20^{\circ} 20.6^{\prime} \\
20^{\circ} 20.6^{\prime}\end{array}$ & $\begin{array}{l}40^{\circ} 56.2^{\prime} \\
40^{\circ} 56.2^{\prime} \\
40^{\circ} 56.2^{\prime} \\
40^{\circ} 56.2^{\prime}\end{array}$ & $\begin{array}{l}35.2 \\
14.9 \\
15.4 \\
11.7\end{array}$ & $\begin{array}{l}516 \\
620 \\
591 \\
512\end{array}$ & $\begin{array}{l}.1973 \\
.0694 \\
.0755 \\
.0656\end{array}$ & $\begin{array}{l}.70471 \\
.70395 \\
.70427 \\
.70382\end{array}$ & $\begin{array}{l}\text { Quartz diorite } \\
\text { Do. } \\
\text { Do. } \\
\text { Do. }\end{array}$ \\
\hline $\begin{array}{r}66 \ldots \\
743-4 \mathrm{~K} \\
743-4 \mathrm{M} \\
743-4 \mathrm{~N}\end{array}$ & $\begin{array}{l}20^{\circ} 19.0^{\prime} \\
20^{\circ} 19.0^{\prime} \\
20^{\circ} 19.0^{\prime} \\
20^{\circ} 19.0^{\prime}\end{array}$ & $\begin{array}{l}40^{\circ} 52.3^{\prime} \\
40^{\circ} 52.3^{\prime} \\
40^{\circ} 52.3^{\prime} \\
40^{\circ} 52.3^{\prime}\end{array}$ & $\begin{array}{l}22.8 \\
44.5 \\
38.2 \\
43.3\end{array}$ & $\begin{array}{l}584 \\
458 \\
402 \\
433\end{array}$ & $\begin{array}{l}.1128 \\
.2812 \\
.2754 \\
.2893\end{array}$ & $\begin{array}{l}.70487 \\
.70623 \\
.70678 \\
.70683\end{array}$ & $\begin{array}{l}\text { Do. } \\
\text { Do. } \\
\text { Do. } \\
\text { Do. }\end{array}$ \\
\hline 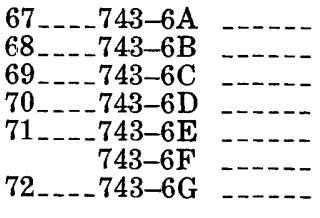 & $\begin{array}{l}19^{\circ} 46.8^{\prime} \\
19^{\circ} 42.3^{\prime} \\
19^{\circ} 44.0^{\prime} \\
19^{\circ} 43.7^{\prime} \\
19^{\circ} 43.3^{\prime} \\
19^{\circ} 43.3^{\prime} \\
19^{\circ} 43.0^{\prime}\end{array}$ & $\begin{array}{l}42^{\circ} 17.4^{\prime} \\
42^{\circ} 18.4^{\prime} \\
42^{\circ} 19.4^{\prime} \\
42^{\circ} 19.8^{\prime} \\
42^{\circ} 21.5^{\prime} \\
42^{\circ} 21.5^{\prime} \\
42^{\circ} 21.8^{\prime}\end{array}$ & $\begin{array}{l}4.87 \\
18.8 \\
28.0 \\
22.8 \\
39.9 \\
11.8 \\
17.3\end{array}$ & $\begin{array}{l}510 \\
408 \\
593 \\
539 \\
448 \\
701 \\
744\end{array}$ & $\begin{array}{l}.0276 \\
.1329 \\
.1366 \\
.1225 \\
.2578 \\
.0486 \\
.0674\end{array}$ & $\begin{array}{l}.70322 \\
.70448 \\
.70443 \\
.70442 \\
.70571 \\
.70333 \\
.70348\end{array}$ & $\begin{array}{c}\text { Trondhjemite } \\
\text { Do. } \\
\text { Quartz diorite } \\
\text { Do. } \\
\text { Do. } \\
\text { Do. } \\
\text { Do. }\end{array}$ \\
\hline $\begin{array}{l}73 \ldots--7411-9 \mathrm{~A} \\
74 \ldots-\ldots-11-9 \mathrm{~B}\end{array}$ & $\begin{array}{l}17^{\circ} 31.0^{\prime} \\
17^{\circ} 30.8^{\prime}\end{array}$ & $\begin{array}{l}43^{\circ} 03.5^{\prime} \\
43^{\circ} 04.1^{\prime}\end{array}$ & $\begin{array}{l}179 \\
62.8\end{array}$ & $\begin{array}{l}131 \\
914\end{array}$ & $\begin{array}{l}3.953 \\
.1986\end{array}$ & $\begin{array}{l}.74638 \\
.70492\end{array}$ & $\begin{array}{l}\text { Granodiorite } \\
\text { Quartz monzonite }\end{array}$ \\
\hline $75 \ldots--7411-9 \mathrm{C} \quad \ldots$ & $17^{\circ} 30.9^{\prime}$ & $43^{\circ} 04.0^{\prime}$ & 121 & 286 & 1.219 & .71404 & Do. \\
\hline $\begin{array}{r}76 \\
77--J-653 \\
78 \\
78- \\
\text { J-669 }\end{array}$ & $\begin{array}{l}19^{\circ} 36.0^{\prime} \\
19^{\circ} 33.0^{\prime} \\
19^{\circ} 31.0^{\prime} \\
19^{\circ} 31.0^{\prime}\end{array}$ & $\begin{array}{l}41^{\circ} 58.5^{\prime} \\
41^{\circ} 57.6^{\prime} \\
41^{\circ} 59.3^{\prime} \\
41^{\circ} 59.3^{\prime}\end{array}$ & $\begin{array}{r}18.5 \\
18.1 \\
19.3 \\
6.6\end{array}$ & $\begin{array}{l}411 \\
400 \\
439 \\
589\end{array}$ & $\begin{array}{l}.1290 \\
.1296 \\
.1258 \\
.0316\end{array}$ & $\begin{array}{l}.70420 \\
.70417 \\
.70407 \\
.70312\end{array}$ & $\begin{array}{l}\text { Quartz diorite } \\
\text { Do. } \\
\text { Do. } \\
\text { Do. }\end{array}$ \\
\hline 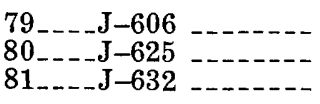 & $\begin{array}{l}19^{\circ} 54.4^{\prime} \\
19^{\circ} 51.3^{\prime} \\
19^{\circ} 52.6^{\prime}\end{array}$ & $\begin{array}{l}41^{\circ} 34.2^{\prime} \\
41^{\circ} 34.6^{\prime} \\
41^{\circ} 34.8^{\prime}\end{array}$ & $\begin{array}{l}16.3 \\
11.5 \\
19.0\end{array}$ & $\begin{array}{l}370 \\
381 \\
394\end{array}$ & $\begin{array}{l}.1277 \\
.0874 \\
.1395\end{array}$ & $\begin{array}{l}.70450 \\
.70401 \\
.70464\end{array}$ & $\begin{array}{l}\text { Do. } \\
\text { Do. } \\
\text { Do. }\end{array}$ \\
\hline $\begin{array}{l}82 \ldots-725-9 \mathrm{~A} \\
83+\cdots\end{array}$ & $\begin{array}{l}19^{\circ} 29.0^{\prime} \\
19^{\circ} 23.6^{\prime}\end{array}$ & $\begin{array}{l}41^{\circ} 37.0^{\prime} \\
41^{\circ} 35.3^{\prime}\end{array}$ & $\begin{array}{l}26.2 \\
23.6\end{array}$ & $\begin{array}{l}376 \\
407\end{array}$ & $\begin{array}{l}.2014 \\
.1686\end{array}$ & $\begin{array}{l}.70552 \\
.70490\end{array}$ & $\begin{array}{l}\text { Do. } \\
\text { Do. }\end{array}$ \\
\hline 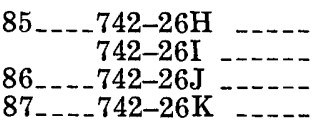 & $\begin{array}{l}20^{\circ} 20.1^{\prime} \\
20^{\circ} 20.1^{\prime} \\
20^{\circ} 19.8^{\prime} \\
20^{\circ} 05.6^{\prime}\end{array}$ & $\begin{array}{l}43^{\circ} 10.0^{\prime} \\
43^{\circ} 10.0^{\prime} \\
43^{\circ} 08.5^{\prime} \\
43^{\circ} 04.0^{\prime}\end{array}$ & $\begin{array}{l}21.7 \\
21.4 \\
13.1 \\
52.1\end{array}$ & $\begin{array}{l}703 \\
798 \\
948 \\
567\end{array}$ & $\begin{array}{l}.0891 \\
.0776 \\
.0401 \\
.2655\end{array}$ & $\begin{array}{l}.70387 \\
.70327 \\
.70329 \\
.70547\end{array}$ & $\begin{array}{l}\text { Do. } \\
\text { Do. } \\
\text { Do. } \\
\text { Do. }\end{array}$ \\
\hline $\begin{array}{r}88 \\
89 \\
8411-7 \mathrm{~A}\end{array}$ & $\begin{array}{l}18^{\circ} 21.7^{\prime} \\
18^{\circ} 24.3^{\prime} \\
18^{\circ} 24.3^{\prime} \\
18^{\circ} 24.3^{\prime} \\
18^{\circ} 23.3^{\prime} \\
18^{\circ} 23.3^{\prime} \\
18^{\circ} 23.3^{\prime} \\
18^{\circ} 23.3^{\prime} \\
18^{\circ} 21.9^{\prime} \\
18^{\circ} 21.9^{\prime} \\
18^{\circ} 28.3^{\prime} \\
18^{\circ} 28.3^{\prime} \\
18^{\circ} 08.2^{\prime} \\
18^{\circ} 08.2^{\prime} \\
18^{\circ} 07.0^{\prime}\end{array}$ & $\begin{array}{l}43^{\circ} 59.5^{\prime} \\
43^{\circ} 45.6^{\prime} \\
43^{\circ} 45.6^{\prime} \\
43^{\circ} 45.6^{\prime} \\
43^{\circ} 46.8^{\prime} \\
43^{\circ} 46.8^{\prime} \\
43^{\circ} 46.8^{\prime} \\
43^{\circ} 46.8^{\prime} \\
43^{\circ} 42.3^{\prime} \\
43^{\circ} 42.3^{\prime} \\
43^{\circ} 44.8^{\prime} \\
43^{\circ} 44.8^{\prime} \\
43^{\circ} 38.5^{\prime} \\
43^{\circ} 38.5^{\prime} \\
43^{\circ} 37.8^{\prime}\end{array}$ & $\begin{array}{l}33.9 \\
18.2 \\
54.4 \\
39.8 \\
23.0 \\
33.4 \\
26.9 \\
19.7 \\
72.7 \\
74.3 \\
43.3 \\
24.4 \\
3.02 \\
2.72 \\
2.8\end{array}$ & $\begin{array}{r}867 \\
1214 \\
858 \\
440 \\
1019 \\
821 \\
862 \\
979 \\
518 \\
508 \\
723 \\
509 \\
636 \\
1294 \\
1048\end{array}$ & $\begin{array}{l}.1131 \\
.0433 \\
.1834 \\
.2622 \\
.0653 \\
.1177 \\
.0901 \\
.0581 \\
.4062 \\
.4236 \\
.1732 \\
.1388 \\
.0138 \\
.0061 \\
.0077\end{array}$ & $\begin{array}{l}.70394 \\
.70314 \\
.70465 \\
.70539 \\
.70351 \\
.70395 \\
.70375 \\
.70347 \\
.70684 \\
.70701 \\
.70457 \\
.70451 \\
.70232 \\
.70242 \\
.70256\end{array}$ & $\begin{array}{l}\text { Granodiorite } \\
\text { Quartz diorite } \\
\text { Granodiorite } \\
\text { Quartz diorite } \\
\text { Do. } \\
\text { Granodiorite } \\
\text { Do. } \\
\text { Quartz diorite } \\
\text { Granodiorite } \\
\text { Do. } \\
\text { Do. } \\
\text { Quartz diorite } \\
\text { Do. } \\
\text { Do. } \\
\text { Trondhjemite }\end{array}$ \\
\hline
\end{tabular}


TABLE 2.-Summary of $R b-S r$ ages and isochron data

[See table 1 for listing of localities]

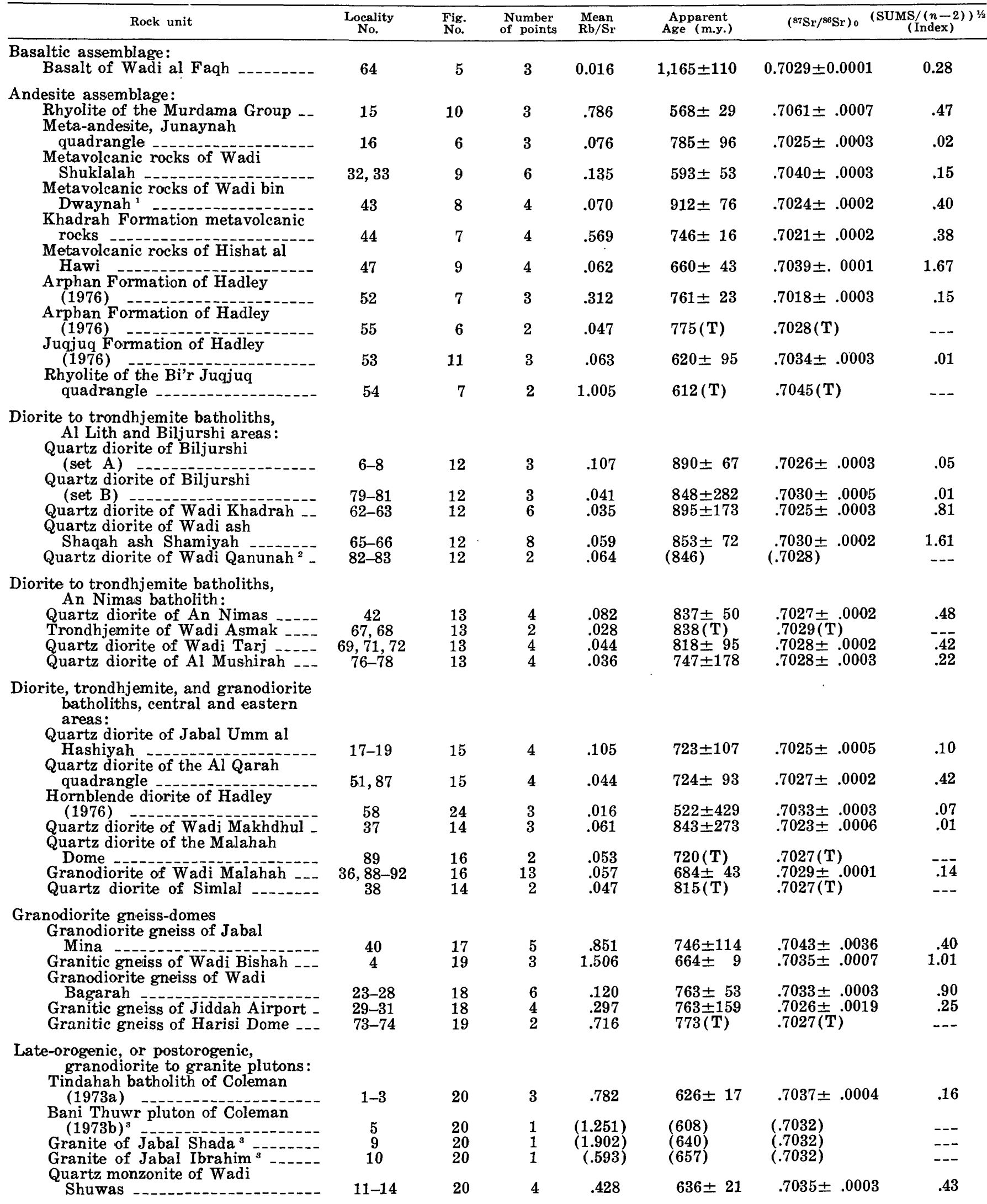


TABLE 2.-Summary of Rb-Sr ages and isochron data-Continued

\begin{tabular}{|c|c|c|c|c|c|c|c|}
\hline Rock unit & $\begin{array}{l}\text { Locality } \\
\text { No. }\end{array}$ & $\begin{array}{l}\text { Fig. } \\
\text { No. }\end{array}$ & $\begin{array}{c}\text { Number } \\
\text { of points }\end{array}$ & $\begin{array}{l}\mathrm{Mean} \\
\mathrm{Rb} / \mathrm{Sr}\end{array}$ & $\begin{array}{l}\text { Apparent } \\
\text { Age (m.y.) }\end{array}$ & $\left({ }^{87} \mathrm{Sr} /{ }^{808} \mathrm{Sr}\right)_{0}$ & ${ }_{(\text {Index }}^{(\text {SUMS } /(n-2))^{3 / 2}}$ \\
\hline \multicolumn{8}{|l|}{$\begin{array}{l}\text { Late-orogenic, or postorogenic, } \\
\text { granodiorite to granite } \\
\text { plutons-Continued }\end{array}$} \\
\hline Quartz monzonite of Jabal Qal _--- & $21-22$ & 21 & 3 & 2.429 & $620 \pm 18$ & $0.7034 \pm 0.0005$ & 0.03 \\
\hline Granodiorite of Wadi Halal & $34-35$ & 22 & 7 & .232 & $643 \pm 20$ & $.7028 \pm .0002$ & .52 \\
\hline Granodiorite of Wadi al Miyah & $45-46$ & 23 & 3 & .789 & $587 \pm 99$ & $.7045 \pm .0030$ & .77 \\
\hline Granodiorite of Wadi Musayrah & $48-50$ & 23 & 7 & .326 & $623 \pm 18$ & $.7033 \pm .0003$ & .42 \\
\hline $\begin{array}{l}\text { Aplite dike, southwestern Al Qarah } \\
\text { quadrangle }\end{array}$ & 51 & 23 & 0 & 252 & 21 (T) & 7000 (T) & \\
\hline & b1 & 23 & 2 & .253 & $621(1)$ & $.6028(1)$ & $\cdots$ \\
\hline $\begin{array}{l}\text { quadrangle }^{2} \\
\text { Quartz monzonite, Bi'r Juqjuq }\end{array}$ & $56-57$ & 25 & 5 & .287 & $(620)$ & $(.7032)$ & --- \\
\hline $\begin{array}{l}\text { quadrangle }{ }^{2} \\
\text { Quartz monzonite of Jabal }\end{array}$ & 59 & 25 & 3 & .476 & $(636)$ & $(.7032)$ & -- \\
\hline Tarban & 61 & 25 & 2 & .589 & $635(\mathrm{~T})$ & $.7031(\mathrm{~T})$ & -- \\
\hline
\end{tabular}

1 Only data points for older isochron summarized here.

2 Data have insufficient spread in $\mathrm{Rb} / \mathrm{Sr}$. Age reported represents model age, using weighted mean of all points and assumed initial ${ }^{87} \mathrm{Sr} /{ }^{\mathrm{se}} \mathrm{Sr}$.

${ }^{3}$ Data are for single point. Age reported represents model age using assumed initial ${ }^{87} \mathrm{Sr} /{ }^{86} \mathrm{Sr}$.

ages in table 2 with cited uncertainties were calculated by least-squares regression. Ages and initial ratios followed by $(\mathrm{T})$ are 2-point "isochrons" and no uncertainties are quoted because their validity cannot be evaluated. Ages shown in parentheses are "model ages," calculated for single samples by assuming the initial ${ }^{87} \mathrm{Sr} /{ }^{86} \mathrm{Sr}$ ratio shown in parentheses. The assumed values are the mean initial ${ }^{87} \mathrm{Sr} /{ }^{86} \mathrm{Sr}$ ratios (here designated $r_{i}$ for convenience) obtained from isochrons of similar rock units. Because of the high degree of uniformity in $r_{i}$ values of a given rock type (table 2 ), the uncertainty attached to this assumption is probably only slightly greater than estimates cited for many of the isochrons themselves (c.a. $<0.0005$ at one standard deviation). As in the case of 2-point "isochrons," uncertainties are not assigned. These data not only provide a chronology for the evolution of the Arabian Shield but also support a plate-tectonic model for that evolution.

\section{BASALTIC ASSEMBLAGE}

Although stratified rocks of the basaltic assemblage occur over large areas of the southern part of the shield, only one locality (loc. 64, fig. 4) has been sampled sufficiently to yield a total-rock Rb-Sr isochron. Basaltic flow rocks (now in greenschist facies) from this locality, near Wadi al Faqh, were very low in rubidium, averaging about $6 \mathrm{ppm}$. For this reason, all analyses of rubidium and strontium were done by isotope dilution. The isochron diagram for the three basalts (fig. 5) indicates an age of $1165 \pm 110 \mathrm{~m} . y$. and $r_{i}$ of $0.7029 \pm 0.0001$. The calculated Index of 0.28 suggests that dispersion of the points is easily within analytical uncertainty.
Rubidium concentrations of the basalts of Wadi al Faqh are similar to those of tholeiitic basalts from either island-arc or ocean-floor environments but are significantly less than those of alkali basalts (Hart and others, 1970). Strontium concentrations, however, are significantly greater than those of oceanfloor basalts and comparable to those of island arcs such as New Britain or Japan. Similar conclusions regarding magma genesis were drawn by Greenwood

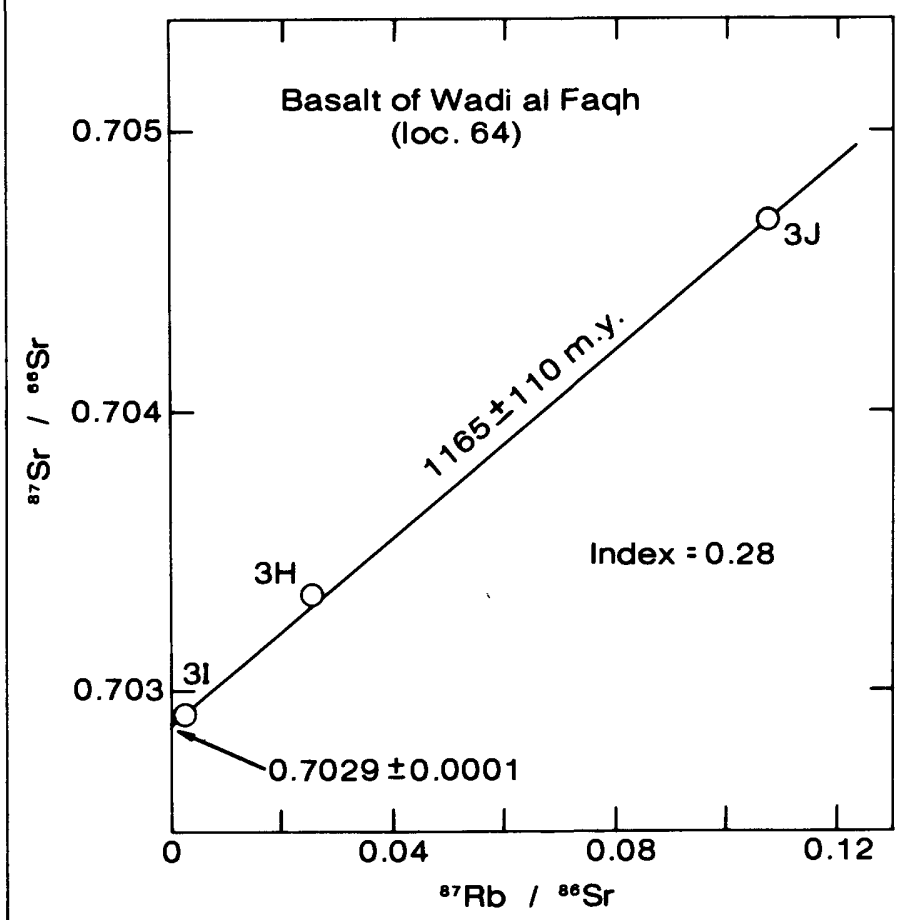

FIGURE 5.-Total-rock Rb-Sr isochron diagram of the basalt of Wadi al Faqh. Sample location is shown on figure 4 and in tables 1 and 2. 
and others (1976) from major-element concentrations in rocks of the basaltic assemblage and by Greenwood and others (1977) from trace-element concentrations.

\section{ANDESITIC ASSEMBLAGE}

Age determinations of volcanic rocks of the andesitic assemblage (table 2) exhibit significant variation. Geologic controls provided by K-Ar minimum ages or $\mathrm{Rb}-\mathrm{Sr}$ ages of plutonic bodies that intrude the volcanic units indicate that some of the apparent ages of fine-grained volcanic strata have been reset by low-temperature events. Five of the units sampled, however, yield ages that are consistent with geologic constraints and are interpreted as times of extrusion.

\section{UNDISTURBED ISOTOPIC SYSTEMS}

Isochron diagrams of data from andesitic strata that reflect closed isotopic systems, shown in figures 6 and 7 , yield ages between 746 and 785 m.y. for four of the five groups. Samples of the Arphan Formation of Hadley (1976) (Halaban Group) from two different localities (locs. 52, 55; tables 1 and 2; fig. 4) yield ages of $761 \pm 23$ m.y. and 775 m.y., although the latter is a two-point "isochron." Metaandesites from the Junaynah (loc. 16) and Khadrah (loc. 44) quadrangles yield ages of $785 \pm 96$ m.y. and $746 \pm 16$ m.y., respectively, with excellent indices of fit (table 2 ). The generally low initial ratios of these groups (0.7018 and 0.7028) support the interpretation of these ages as times of eruptions, although the low $\mathrm{Rb} / \mathrm{Sr}$ ratios of some of the samples would

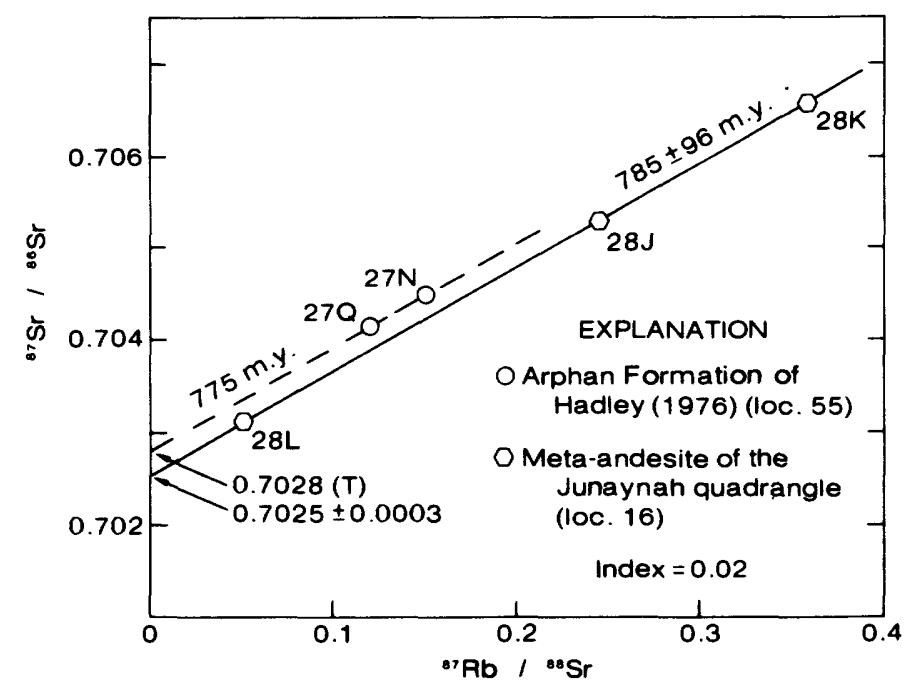

Figure 6.-Total-rock Rb-Sr isochron diagram of the Arphan Formation of Hadley (1976) and of the meta-andesite of the Junaynah quadrangle. Sample locations correspond to those in figure 4 and table 1.

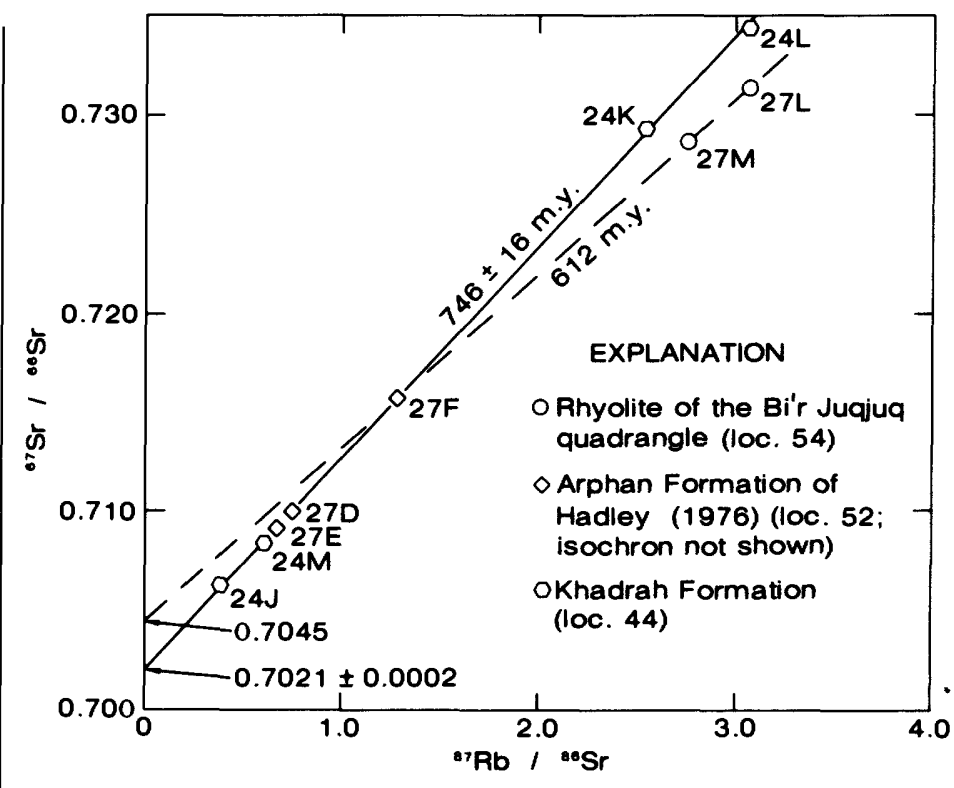

FIGURE 7.-Total-rock $\mathrm{Rb}-\mathrm{Sr}$ isochron diagram of the Khadrah Formation metavolcanic rocks (Greenwood, 1979b), rhyolite of the Bi'r Juqjuq quadrangle, and the Arphan Formation of Hadley (1976). Where isochrons are so nearly simultaneous as to add confusion to the diagram, one or more of them may have been omitted. Data for all isochrons are shown in table 2. Sample locations are shown in figure 4 and listed in table 1.

still permit an interpretation of isotopic homogenization of slightly older volcanic rocks as an undeniable alternative. Even if the calculated ages were reset, however, the data do not permit the times of eruption to be much older.

The youngest andesitic unit studied, discussed here as the rhyolite of the Bi'r Juqjuq quadrangle (loc. 54), was mapped as the youngest unit of the Arphan Formation of Hadley (1976) and considered to be part of the Halaban Group (Hadley, 1976). The apparent age of the strata, as determined from only two points, is 612 m.y., however, considerably younger than the andesitic units upon which it rests (locs. 52 and 55; figs. 6, 7). Hadley (1976) mapped sills of a similar rhyolite porphyry that intrude the older units nearby; this relationship suggests that the rhyolite may be a late-orogenic or postorogenic shallow intrusive or ash flow related to the sills. Although the high $r_{i}$ of 0.7045 for this "isochron" (fig. 7) would permit interpretation of the age as having been reset by metamorphism, the high $\mathrm{Rb} / \mathrm{Sr}$ ratio of the rocks would require an $r_{i}$ below meteorite values (less than 0.699 ) in order for the rhyolite to have been contemporaneous with the other units of the Arphan Formation studied (ca. 770 m.y.). We now consider this rhyolite to be a younger, post- 
metamorphic unit, possibly equivalent to the Shammer Rhyolite of Brown and Jackson (1960) or to the Shammer Formation of Delfour (1970).

\section{DISTURBED ISOTOPIC SYSTEMS}

Units of the andesitic assemblage discussed although folded and faulted are less deformed than many sequences of andesitic strata within the Arabian Shield. The discussed localities are confined to the central and eastern parts of the area studied and probably represent younger parts of the assemblage (Halaban Group). Older units of the assemblage, however, are exposed to the west, such as in Wadi bin Dwaynah, where metavolcanic rocks mapped as Jiddah Group by Greenwood (1979a) are part of a large roof pendant in the An Nimas batholith. This batholith is one of the older quartz diorite to trondhjemite batholiths of the southern part of the shield and predates much of the andesitic assemblage (see fig. 2). A $\mathrm{Rb}-\mathrm{Sr}$ isochron diagram (fig. 8) of all samples from the Wadi bin Dwaynah locality (loc. 43, fig. 4) suggests open-system behavior in some samples. The data points define two intersecting linear arrays that yield apparent ages of $912 \pm 76$ m.y. $\left(r_{i}=0.7024 \pm 0.0002\right)$ and $641 \pm 28$ m.y. $\left(r_{i}=0.7030 \pm 0.0002\right)$. All seven samples fit one array or the other, with Index Values of 0.40 and 0.42 for the older and younger groups, respectively. As a whole, however, the data set yields a composite age of $669 \pm 25 \mathrm{~m} . \mathrm{y} ., r_{i}$ of $0.7030 \pm 0.0001$, and an Index of 1.67. The Index is equal to the highest obtained in this study and exceeds the cutoff of 1.58, suggesting geologic error. Where it intrudes these units of the andesitic assemblage near Wadi bin Dwaynah, the An Nimas batholith yields an age of $837 \pm 50$ m.y., a minimum age for the metavolcanic rocks. The apparent age obtained from the composite of all Wadi bin Dwaynah samples is significantly younger than the age of the batholith at all confidence levels up to 99.7 percent, and the strontium isotopic system of the metavolcanic rocks is considered disturbed.' The 912-m.y. apparent age obtained from one grouping of samples would be consistent with the $837 \pm 50$ m.y. age of the batholith, but this grouping cannot be justified by any independent criteria. We suggest that the $912 \pm 76$ m.y. age may represent the time of extrusion of the strata, but we are aware of the tenuous nature of this conclusion.

Fine-grained metavolcanic rocks sampled near Hishat al Hawi (loc. 47, fig. 4) yield an elevated Index of fit and young apparent age of $660 \pm 43$ m.y. (fig. 9). As do the Indices for the Wadi bin Dwaynah rocks, the Index of 1.67 itself indicates geologic error; the clearly open-system behavior of some of the Wadi bin Dwaynah rocks that yield an identical age and index of fit leaves open the possibility of partial or complete isotopic homogenization of strontium in the Hishat al Hawi strata as well. The $r_{i}$ of these rocks of $0.7039 \pm 0.0001$ is high relative to other andesitic units but is not sufficiently elevated to indicate unambiguously a resetting of isotopic systems. Because the strata are correlated with the

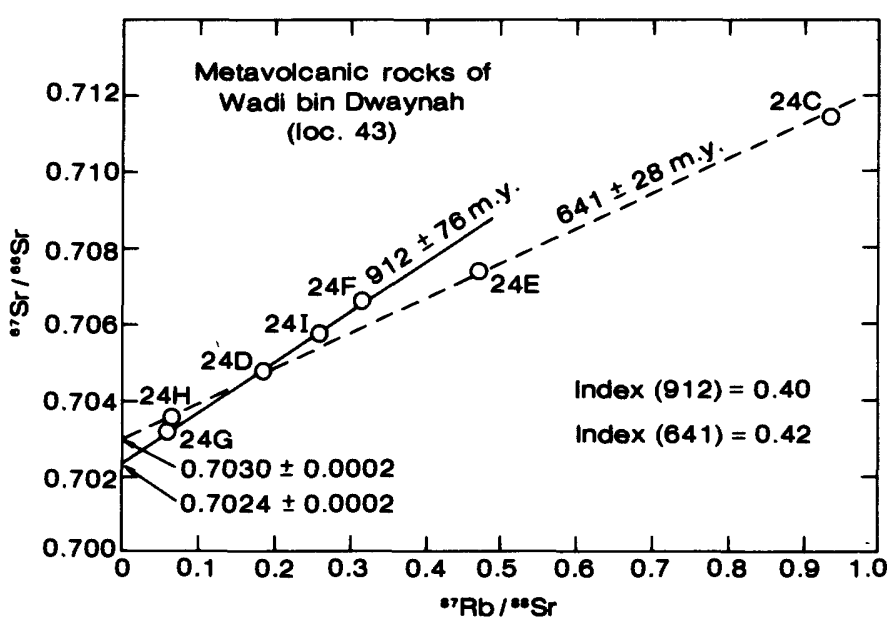

FIGURE 8.-Total-rock $\mathrm{Rb}-\mathrm{Sr}$ isochron diagram of the metavolcanic rocks of Wadi bin Dwaynah. See figure 7 for explanation of isochron data.

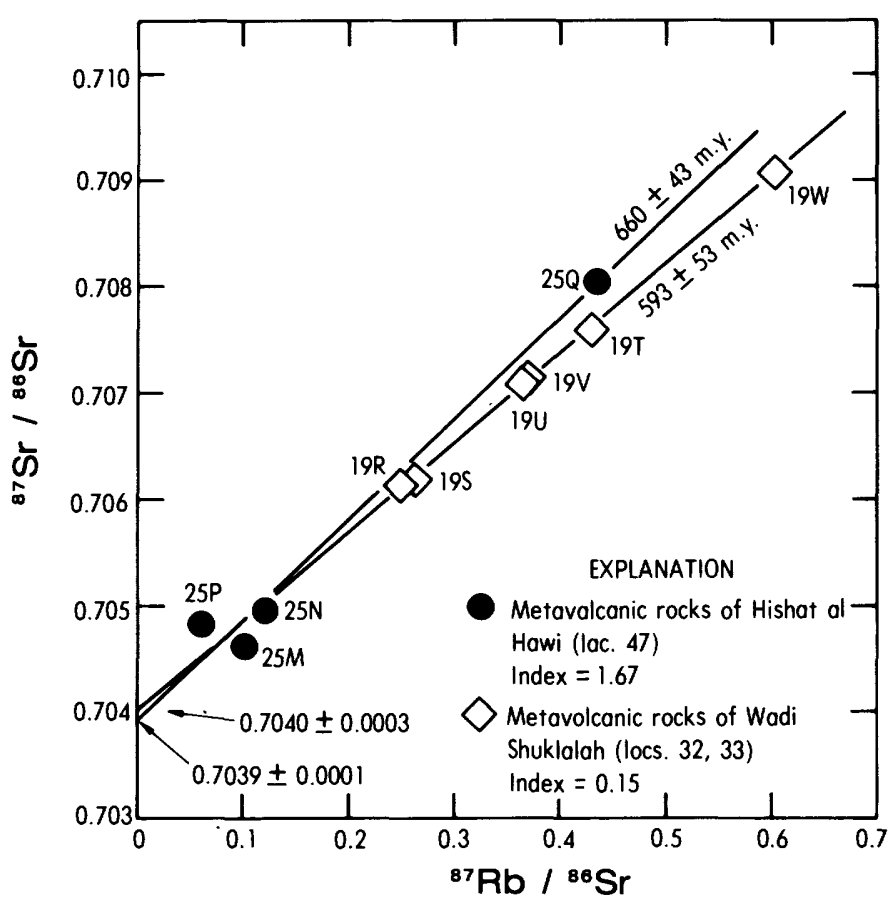

FIGURE 9.-Total-rock Rb-Sr isochron diagram of metavolcanic rocks of Hishat al Hawi and Wadi Shuklalah. See figure 7 for explanation of isochron data. 
meta-andesite of the Junaynah quadrangle (loc. 16), which yields an age of $785 \pm 96$ m.y., however, we conclude that the $660-\mathrm{m} . \mathrm{y}$. age does not represent the time of extrusion of the metavolcanic rocks of Hishat al Hawi.

Several other sequences of fine-grained metavolcanic strata yield isochrons having acceptable indices of fit (Brooks and others, 1972) that probably reflect the time of metamorphism rather than of extrusion. The metavolcanic rocks of Wadi Shuklalah (locs. 32, 33, fig. 4), plotted in figure 9, are of this type. These strata, found in the southeastern part of the shield, are deformed and metamorphosed by a quartz diorite to granodiorite body (granodiorite of Wadi Halal) that yields an age of $643 \pm 20$ m.y., but the total-rock isochron for the metavolcanic rocks yields an age of $593 \pm 53$ m.y., $r_{i}$ of $0.7040 \pm 0.0003$, and Index of 0.15 . Although the age difference is significant only at confidence levels less than 62.3 percent, the geologic control and slightly elevated $r_{i}$ suggest a metamorphic age even though the original ash-flow tuff fabric of some units is nearly undisturbed.

Fine-grained rhyolite within young strata of the andesitic assemblage (the Murdama Group) in the Jabal Yafikh quadrangle (loc. 15) also appears to yield a reset total-rock isochron, with an age of $568 \pm 29$ m.y. (fig. 10). Although the strata are tightly folded, metamorphic effects are not detectable in the volcanic rocks studied. Deformation appears to increase with proximity to one of the major shear zones of the Najd fault system (Brown and Jackson, 1960). Discordant K-Ar ages on hornblende are reported by Fleck and others (1976) for quartz diorite upon which the strata were deposited, but no geologic control of minimum age is available within the area. The interpretation of the age as reset is based on the elevated $r_{i}$ of $0.7061 \pm 0.0007$ but must be considered tenuous because of the lack of independent control.

Andesitic strata in the Bi'r Juqjuq quadrangle assigned to the Juqjuq Formation of Hadley (1976) (Halaban Group) are intruded by the quartz monzonite of Jabal Tarban, which yields an apparent age of about 635 m.y. (table 2, loc. 61). As in the Jabal Yafikh area (loc. 15), the stratified units are folded, but metamorphism is not represented by petrographically detectable textural or mineralogical changes. Where sampled (loc. 53), the Juqjuq Formation contains detrital, volcaniclastic, and volcanic units. Three andesites from the unit yield a totalrock isochron age of $620 \pm 95 \mathrm{~m} . \mathrm{y} ., r_{i}$ of $0.7034 \pm$ 0.0003 , and an Index of 0.01 , indicating extremely low dispersion about the isochron (fig. 11). All have very fine grained mesostases, although two samples from these andesites contain coarse phenocrysts. The quartz monzonite of Jabal Tarban truncates both bedding and fold axes in the Juqjuq Formation, indicating a late-deformation or postdeformation emplacement. The apparent age of the intruded strata is essentially the same as that of the intrusive body, raising the possibility of isotopic homogenization during intrusion. The $r_{i}$ of the andesitic unit

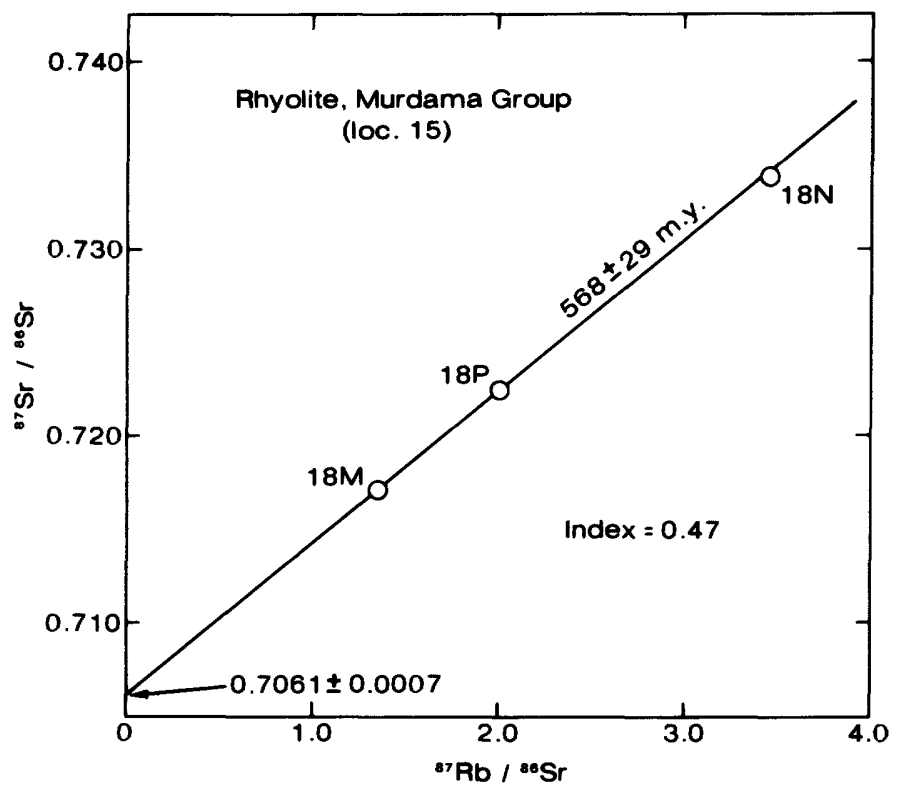

FIgURE 10.-Total-rock $\mathrm{Rb}-\mathrm{Sr}$ isochron diagram of the rhyolite of the Murdama Group. See figure 7 for explanation of symbols and isochron data.

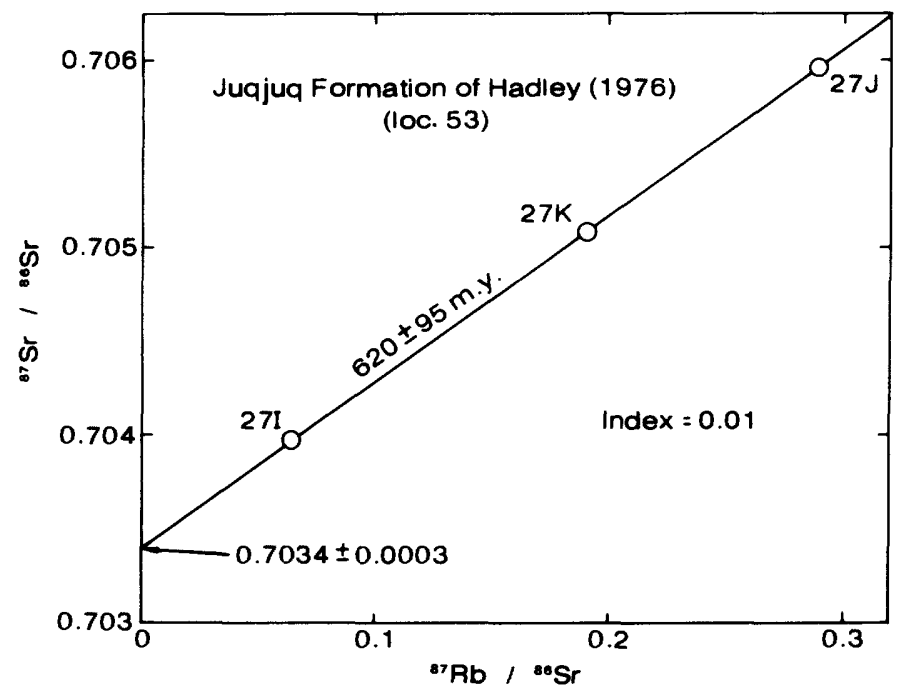

FigURE 11.-Total-rock Rb-Sr isochron diagram of the Juqjuq Formation of Hadley (1976). See figure 7 for explanation of isochron data. 
(0.7034) is not significantly elevated to support a homogenization hypothesis, however, as magmas related to the late-orogenic or postorogenic plutonism commonly have values in that range. $\mathrm{Rb} / \mathrm{Sr}$ ratios of the young plutons, however, are commonly much higher than the 0.063 value (table 2) from the Juqjuq Formation. Hadley (1976) considered the Juqjuq to be older than his Arphan Formation, determined here to be 760-775 m.y. For these reasons, we interpret the apparent age obtained from these strata to be reset but consider this assignment tentative.

Ages interpreted as times of extrusion of volcanic units of the andesitic assemblage range from 612 to 912 m.y. This range of ages does not represent magma generation of constant rate, common source, or similar mechanism, but rather includes a variety of volcanic and volcaniclastic units whose average composition is andesitic. The majority of ages, probably representing as much as 50 percent of the total volume of andesitic strata, are between 740 and 790 m.y. In the southern part of the shield, andesitic units younger than about 700 m.y. probably represent a volumetrically small proportion of the assemblage. Some of the less deformed strata, as well as some of the most deformed ones, yield ages younger than plutonic units that intrude them, indicating disturbance and, commonly, rehomogenization of the strontium isotopic systems within them. The timing of this disturbance is inadequately known. The data indicate repeated, possibly episodic, deposition and metamorphism of andesitic strata over broad areas of the Arabian Shield and severely limit correlation by rock type and degree of deformation. Conversely, the evidence of disturbance of isotopic systems in these strata emphasizes the tenuous nature of interpretation of isotopic ages in fine-grained rocks without additional geologic and geochronologic control.

\section{FOLIATED DIORITE TO TRONDHJEMITE BATHOLITHS}

Plutonic rocks represent about 40 percent of rocks exposed in the Arabian Shield (Greenwood and Brown, 1973). In the western part of the shield, major batholiths range in composition from diorite to trondhjemite, but granodiorite increases in abundance eastward. In the western and southern parts of the area (fig. 3), dioritic batholiths represent 60-80 percent of the intrusive units. In the eastern part, granodiorite gneiss and younger granitic rocks are more abundant. $\mathrm{Rb}-\mathrm{Sr}$ total-rock isochron ages of diorite to trondhjemite bodies provide the most complete and reliable chronology of the evolution of the southern part of the Arabian Shield. Field evidence, particularly in the Biljurshi and al 'Aqiq quadrangles (Greenwood, 1975a, b), demonstrates repeated andesitic volcanism, volcaniclastic sedimentation, dioritic intrusion, deformation, and erosion. Ages of dioritic bodies shown in table 2 support a model of continued, though possibly episodic, igneous activity between about 900 and 680 m.y. ago.

The oldest plutonic rocks studied occur in the $\mathrm{Al}$ Lith and Biljurshi areas (figs. 3 and 4), where they intrude rocks of both the basaltic and andesitic assemblages. Five suites of samples from these areas were studied; $\mathrm{Rb}-\mathrm{Sr}$ data are presented in tables 1 and 2 and figure 12. Quartz diorites of Wadi Khadrah and Wadi ash Shaqah ash Shamiyah (locs. $62,63,65$, and 66) in the area of Al Lith yield ages of $895 \pm 173 \mathrm{~m} . \mathrm{y}$. and $853 \pm 72 \mathrm{~m} . \mathrm{y}$. and $r_{i}$ values of $0.7025 \pm 0.0003$ and $0.7030 \pm 0.0002$, respectively. $\mathrm{Rb} / \mathrm{Sr}$ values for these rocks are very low, rubidium concentrations averaging about $25 \mathrm{ppm}$. Index values of 0.81 and 1.61 are larger than many values

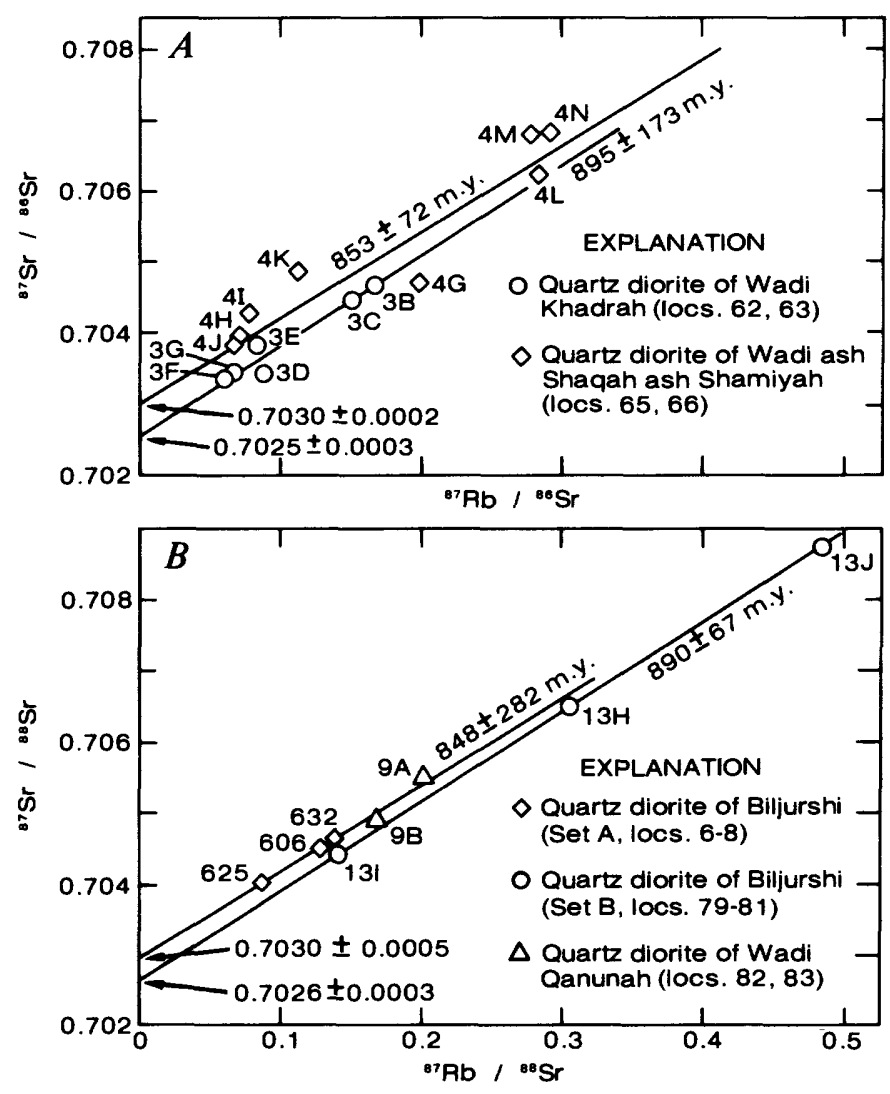

Figure 12.-Total-rock $\mathrm{Rb}-\mathrm{Sr}$ isochron diagrams. $A$, Al Lith area, quartz diorities of Wadi Khadrah and Wadi ash Shaqah ash Shamiyah. $B$, Biljurshi area, quartz diorites of Biljurshi and Wadi Qanunah. Data for all isochrons are shown in table 2. Sample locations are shown in figure 4 and listed in table 1. 
obtained from the diorite units studied, although neither is significantly above the 1.58 cutoff suggested for distinguishing between analytical and geologic error. Data shown in figure 12, however, reveal the much greater scatter of these sets when compared with other sets reported here, and this is reflected in the uncertainties in the apparent ages.

Data for samples from the Biljurshi batholith yield ages similar to those from the Al Lith area, although the intrusive complexes are spatially distinct. Quartz diorite of Biljurshi (locs. 6-8 and 79-81), mapped by Greenwood (1975a) as older quartz diorite, intrudes units of both the basaltic and andesitic assemblages and generally is cut by the major northor northeast-trending fracture systems. Other exposures, however, indicate that some of these faults formed prior to diorite emplacement and were truncated by the plutons. The presence within the fracture system of faults older than the diorite and others that are younger indicates contemporaneous deformation and dioritic intrusion. The ages indicated by the two data sets, $890 \pm 67$ m.y. and $848 \pm 282 \mathrm{~m} . \mathrm{y}$. and $r_{i}$ values of $0.7026 \pm 0.0003$ and $0.7030 \pm 0.0005$, are almost identical with those of the Al Lith samples. In contrast with the Al Lith data, however, the Biljurshi sets show extraordinarily linear arrays with extremely low Index values. Based solely on dispersion of the data, uncertainties calculated for the first and second groups, respectively, are 3 and $4 \mathrm{~m} . \mathrm{y}$. In the southern part of the Biljurshi batholith, the quartz diorite of Wadi Qanunah (locs. 82, 83) yields similar isotopic results, but the two samples from this body are similar in $\mathrm{Rb} / \mathrm{Sr}$ ratio. Assuming an initial ratio of 0.7028 , the average of the other Biljurshi quartz diorites, an apparent age of $846 \mathrm{~m} . \mathrm{y}$. is calculated for this body.

Diorite to trondhjemite bodies of the An Nimas batholith (Greenwood, 1979a; Anderson, 1977) fall into two age groups. The older group, which represents the oldest plutonic rocks of that region, yields ages of 818 to 838 m.y. (locs. $42,67-69,71,72$ ), as shown in figure 13. The quartz diorite of An Nimas (loc. 42) intrudes older units of the andesitic assemblage; at Wadi bin Dwaynah, the metavolcanic rocks yielded an age of $912 \pm 76 \mathrm{~m} . \mathrm{y}$. The $837 \pm 50$ m.y. age (table 2) of the quartz diorite body is consistent with the age interpretation discussed for the metavolcanic rocks, thereby making improbable an alternative hypothesis of a contact metamorphic origin for the 912 m.y. isochron. Quartz diorites in the batholith in the Wadi Tarj quadrangle (Anderson, 1977) yield ages and $r_{i}$ values similar to those of rocks near An Nimas. Trondhjemite of Wadi Asmak

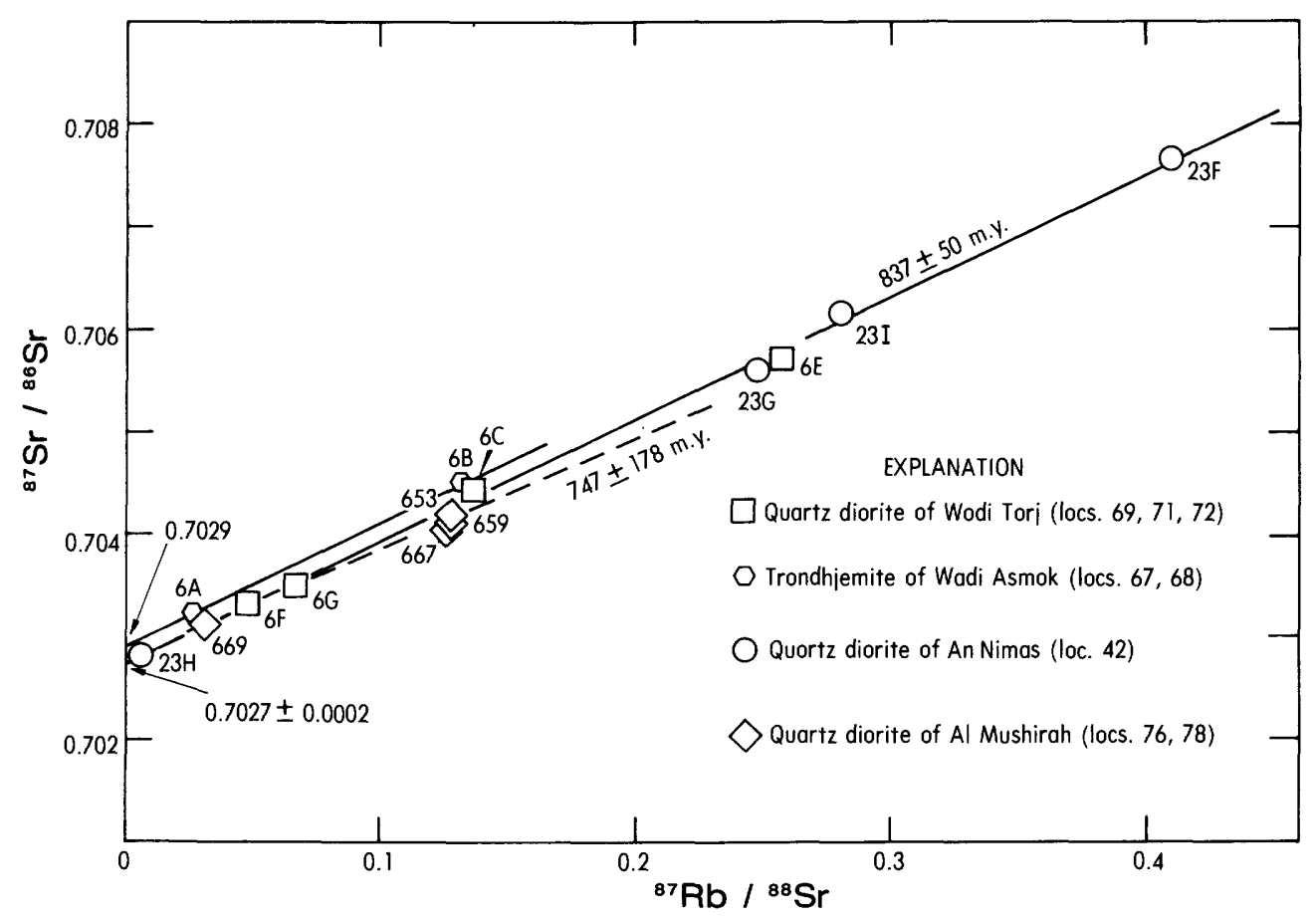

FIGURE 13.-Total-rock Rb-Sr isochron diagram of the quartz diorites of An Nimas, Wadi Tarj, and Al Mushirah and the trondhjemite of Wadi Asmak, An Nimas batholith. See figure 12 for explanation of isochron data. 
(locs. 67, 68) yields a two-point "isochron" age of 838 m.y.; the quartz diorite of Wadi Tarj (locs. 69, $71,72)$, which is closely related to the trondhjemite, defines an isochron of $818 \pm 95$ m.y., with an $r_{i}$ of $0.7028 \pm 0.0002$. In the eastern part of the $\mathrm{Al}$ 'Aqiq (Greenwood, 1975b) and Biljurshi (Greenwood, 1975a) quadrangles, the younger group of dioritic units of the An Nimas batholith intrude strata of the andesitic assemblage (the Ablah Group of Greenwood, 1975b) that were deposited nonconformably on the Biljurshi batholith. One of these, the quartz diorite of Al Mushirah (locs. 76-78), though petrologically indistinguishable from older diorites, yields an age of $747 \pm 178$ m.y. and $r_{i}$ of $0.7028 \pm$ 0.0003 (fig. 13). The age of the younger unit does not differ significantly, however, from that of the quartz diorite of An Nimas (loc. 42) at levels of confidence above 37 percent because three of the four data points have nearly identical $\mathrm{Rb} / \mathrm{Sr}$ ratios.

Dioritic complexes in the central and eastern parts of the area studied yield results similar to those for the Biljurshi and An Nimas batholiths, although ages are commonly younger. Data presented in table 2 define an age range from 522 to 843 m.y., although the youngest age is not considered representative of a time of emplacement. The oldest ages of plutonic rocks from the eastern parts of the region are part of the Wadi Tarib batholith in the Wadi Wassat and Wadi Malahah quadrangles (Greenwood, 1979c, d), where they intrude units of the andesitic assemblage. The diorite complex near Wadi Makhdhul was found to be a composite of plutons of several different ages. The oldest of these probably represents the earliest phase of plutonism in the eastern part of the Arabian Shield, yielding an age of $843 \pm 273$ m.y. and $r_{i}$ of $0.7023 \pm 0.0006$ (loc. 37, fig. 14). Data from diorite and trondhjemite of the Wadi Tarib batholith sampled west of this locality in the southern part of the Wadi Malahah quadrangle (locs. 93, 94) show greater dispersion, as might be expected from the extremely low $\mathrm{Rb}$ content (table 1 ), but fall on the same isochron as those of the Wadi Makhdhul samples (fig. 14). When these three points are included, the isochron age and $r_{i}$ are virtually unchanged, $846 \pm 82$ m.y. and $0.7023 \pm 0.0001$, but their uncertainties are greatly reduced. The Index changes from 0.01 to 0.61 , reflecting the increased scatter, but still indicates that the dispersion is well within the range of analytical variation. Quartz diorite from the area of Simlal, south of Wadi Wassat in the Wadi Qatan area (loc. 38, figs. 4, 14), yields an apparent age of 815 m.y. but is represented by only two points and consequently a large uncertainty.

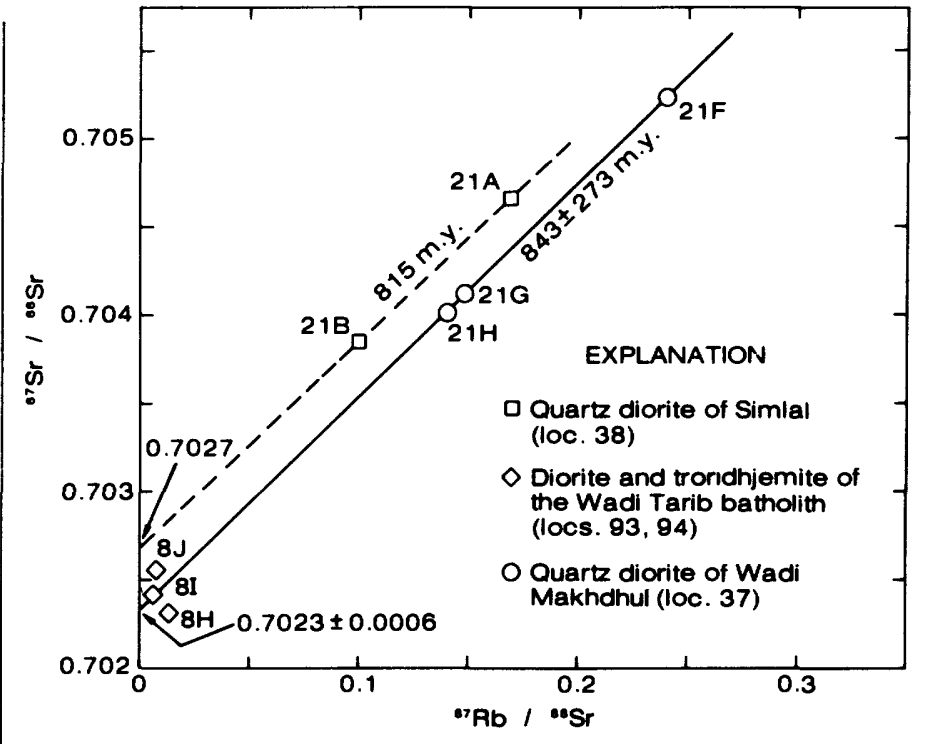

FIgURE 14.-Total-ro:k Rb-Sr isochron diagram of the quartz diorites of Wadi Makhdhul and Simlal and the diorite and trondhjemite of the Wadi Tarib batholith, Wadi Malahah quadrangle. See figure 12 for explanation of isochron data.

The Simlal data do not fit the Wadi Makhdhul diorite isochron (fig. 14) but fall above that line, yielding an $r_{i}$ of 0.7027 . The quartz diorite of the Simlal area is thought to postdate ore deposits in the andesitic assemblage in the Wadi Qatan area (Dodge and Rossman, 1975). Because of its significance for models of evolution of ore deposits, we cannot overemphasize the uncertainty of the two-point "isochron."

Most batholithic units sampled in the central and eastern parts of the area of study yield ages significantly younger than the $815-850$ m.y. range and ${ }^{87} \mathrm{Sr} /{ }^{86} \mathrm{Sr}$ initial ratios greater than 0.7023 . Many of these units have more siliceous phases, ranging from quartz diorite to granodiorite with significant amounts of potassium feldspar and $\mathrm{Rb} / \mathrm{Sr}$ ratios 10-50 times those of the older diorites. Dioritegranodiorites samples from two different units east of Bishah that intrude andesitic units, quartz diorites of Jabal Umm al Hashiyah (locs. 17-19) and southwestern Al Qarah quadrangle (locs. 51, 87), yield ages of $723 \pm 107$ m.y. and $724 \pm 93$ m.y., as shown in figure 15. Initial ratios obtained are $0.7025 \pm 0.0005$ and $0.7027 \pm 0.0002$, respectively. Strata intruded include those of the andesitic assemblage (loc. 16, table 2) that yield an age of $785 \pm 96$ m.y. and represent the oldest unit exposed in the Bishah area.

Diorite and granodiorite units in the Wadi Malahah quadrangle show the greatest age range within the Arabian Shield. As stated, trondhjemite 
and hornblende diorite in the southernmost part of the quadrangle represent very primitive units consistent with the 843 m.y. age of the Wadi Makhdhul diorites. Results from other units including the quartz diorite and granodiorite plotted in figure 16, define significantly different isochrons. Quartz diorite to granodiorite gneiss forms a somewhat sigmoidal east-west-trending body in the Wadi Malahah and western Wadi Wassat quadrangles (fig. 3). North of this body, quartz diorite petrologically similar to the quartz diorite of Wadi Makhdhul is cut by dikes of the granodiorite. These units form the southern margin of a large structural high called the Malahah dome by Greenwood (1979d). Although the regional structure may be domical, a domical foliation is not obvious in the individual plutonic

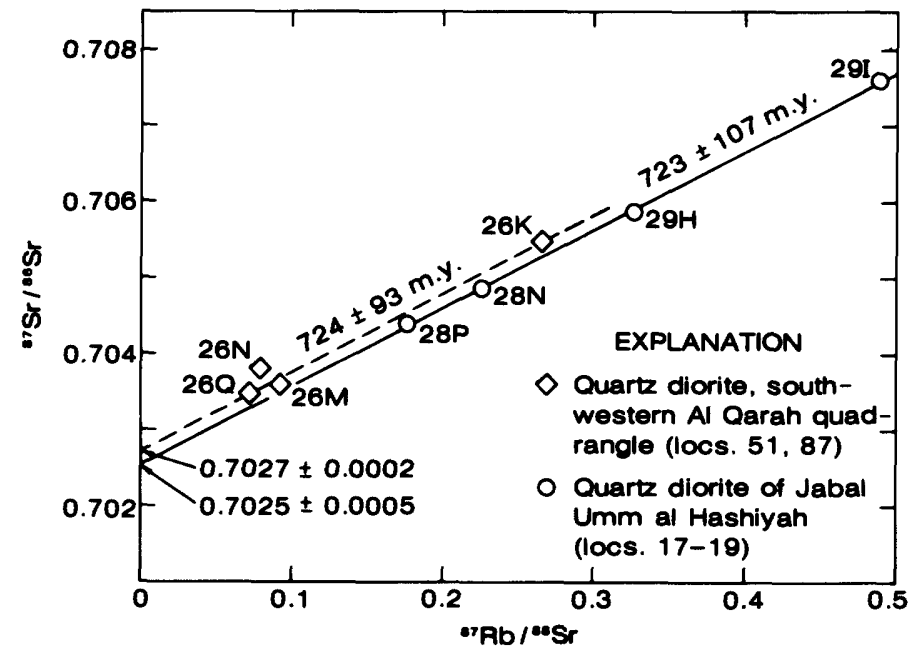

FIGURE 15.-Total-rock $\mathrm{Rb}$-Sr isochron diagram of the quartz diorites of Jabal Umm al Hashiyah and the southwestern Al Qarah quadrangle. See figure 12 for explanation of isochron data.

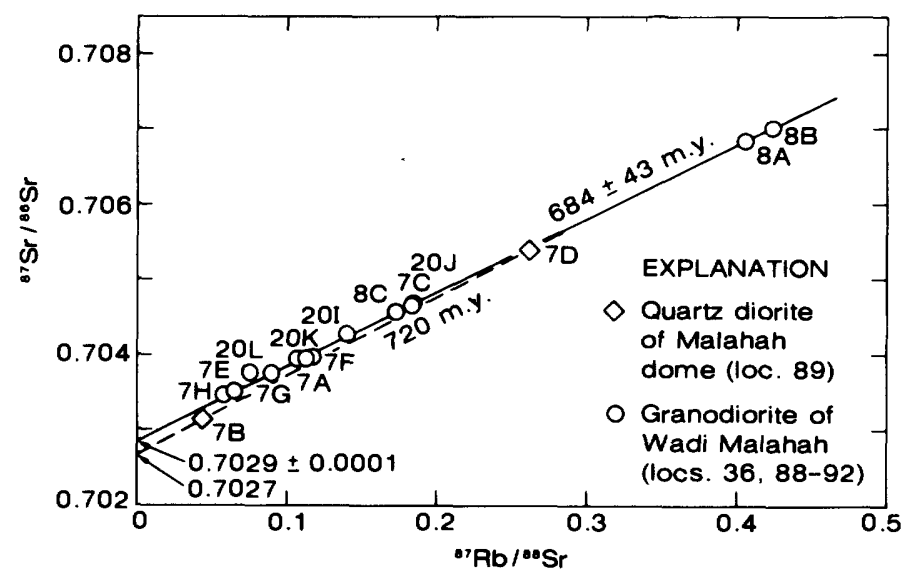

Figure 16-Total-rock $\mathrm{Rb}-\mathrm{Sr}$ isochron diagram of the quartz diorite of Malahah dome and granodiorite of Wadi Malahah. See figure 12 for explanation' of isochron data. units as it is in many of the smaller gneiss domes. The younger of the two major units of the dome, referred to here as the granodiorite of Wadi Malahah, is represented by 13 samples (locs. 36, 88-92) that define an excellent isochron (fig. 16). The age of the unit is $684 \pm 43 \mathrm{~m} . \mathrm{y}$., with an $r_{i}$ of 0.7029 \pm 0.0001 and Index of 0.14 , making this body one of the youngest deformed units studied. The older phase of the dome, the quartz diorite, is represented by only two samples (loc. 89), and although the two differ greatly in $\mathrm{Rb} / \mathrm{Sr}$ ratio, the uncertainties in age and initial ratios easily include those of the younger granodiorite. The apparent age of the quartz diorite is 720 m.y., with an $r_{i}$ of 0.7027 (fig. 16). Although the uncertainties cannot be evaluated, the similiarity in age and $r_{i}$ to those of quartz diorite-granodiorite bodies in the Bishah area (table 2; fig. 15) offers additional support to the results.

Diorite-trondhjemite batholithic complexes occur in a northwest-trending en echelon pattern in the southwestern part of the shield. These bodies range in age from about 750 to $900 \mathrm{~m} . \mathrm{y}$. Diorite-granodiorite complexes are more common in the central and eastern parts of the region than diorite-trondhjemite. They range in age from about 680 to 750 m.y. $\mathrm{Rb} / \mathrm{Sr}$ ratios of the dioritic phases of these two groups are similar, but the granodiorite units show marked increases in $\mathrm{Rb} / \mathrm{Sr}$ relative to the diorites, whereas trondhjemites are characterized by sharp decreases in both $\mathrm{Rb}$ and $\mathrm{Sr}$ from diorite values but with little difference in the $\mathrm{Rb} / \mathrm{Sr}$ ratio.

\section{GRANODIORITE GNEISS DOMES}

Total-rock $\mathrm{Rb}-\mathrm{Sr}$ isochrons were obtained from five deformed granodiorite to granite gneiss domes whose foliations are generally concordant with their intrusive margins. The granodiorite gneiss of Jabal Mina (loc. 40) intrudes a highly deformed amphibolite-grade sequence of the andesite assemblage south and east of An Nimas. Data obtained from the gneiss define a $746 \pm 114$ m.y. isochron (fig. 17), with an $r_{i}$ of $0.7043 \pm 0.0036$ and Index of 0.40 . At the scale of figure 3, the Jabal Mina body is shown as contiguous with the granodiorite gneiss of Wadi Bagarah (locs. 23-28), whereas small pendants of the andesitic assemblage or diorite of the An Nimas batholith actually separate the two. The age of the Wadi Bagarah body was reported earlier by Fleck and others (1973) as 759 m.y. The effects of additional analyses and revised ${ }^{87} \mathrm{Rb}$ decay constants offset each other such that all data for the gneiss now yield an isochron age of $763 \pm 53$ m.y., with an $r_{i}$ of $0.7033 \pm 0.0003$ and Index of 0.90 (fig. 18). Because 


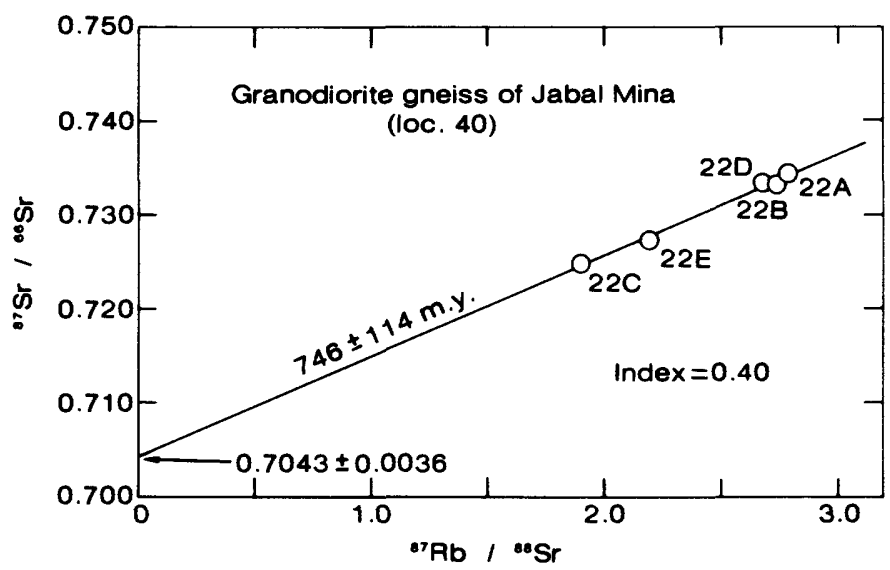

RIGURE 17.-Total-rock $\mathrm{Rb}-\mathrm{Sr}$ isochron diagram of the granodiorite gneiss of Jabal Mina. Data for all isochrons are shown in table 2. Sample locations are shown in figure 4 and listed in table 1.

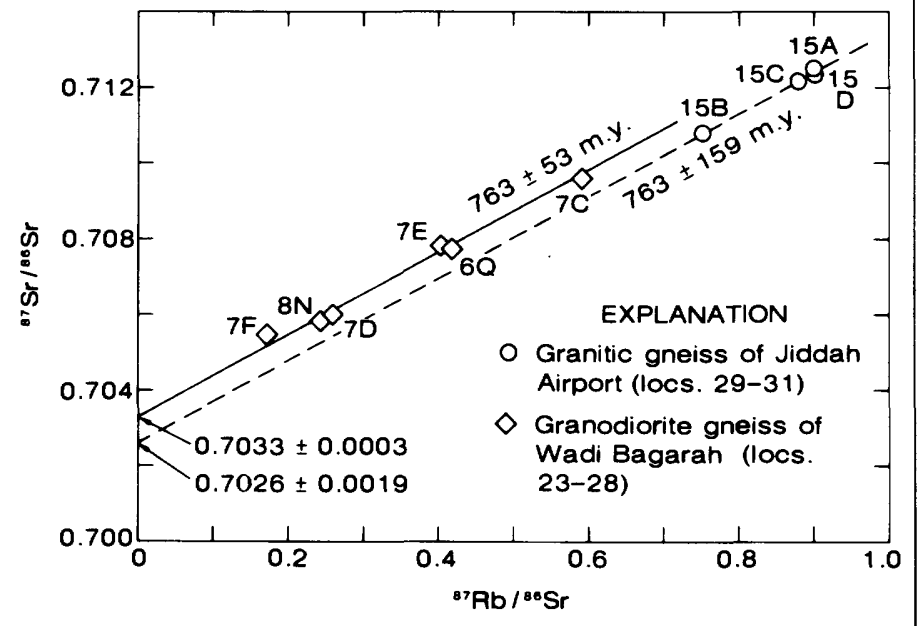

FIgURE 18.-Total-rock Rb-Sr isochron diagram of the granodiorite gneiss of Wadi Bagarah and the granitic gneiss of Jiddah Airport. See figure 17 for explanation of isochron data.

the $\mathrm{Rb} / \mathrm{Sr}$ ratios of the Jabal Mina and Wadi Bagarah bodies differ significantly (table 2), reflecting the lower content of potassium feldspar in the Wadi Bagarah body, the two are considered separate intrusions. The similar ages, tectonic styles, and twomica mineralogy, however, suggest a similar origin.

Samples of biotite-muscovite granitic gneiss exposed $10 \mathrm{~km}$ east of Jiddah were analyzed previously by L. T. Aldrich, quoted by Brown and others (1962), who reports a $\mathrm{Rb}-\mathrm{Sr}$ apparent age on biotite of 1,050 m.y. Brown $(1970,1972)$ referred to these rocks and more widespread exposures of granodiorite and quartz diorite near Mecca as the oldest radiometrically dated units in the Arabian Shield. The rocks exposed near Jiddah, here called the granite gneiss of Jiddah Airport, were resampled by us for whole-rock Rb-Sr analysis (locs. 29-31). Results from these samples yield a whole-rock isochron age of $763 \pm 159$ m.y., an $r_{i}$ of $0.7026 \pm 0.0019$, and Index of 0.25 . These data are inconsistent with an age of 1,050 m.y., at all levels of confidence up to 92.9 percent. They do not invalidate ages reported for dioritic rocks closer to Mecca but suggest a greater similarity to two-mica granitic rocks such as those of Wadi Bagarah or Jabal Mina.

The granite gneiss of Harisi Dome in the southwestern part of the Wadi 'Atf quadrangle (locs. 73, 74 ) is represented by only two samples and the uncertainties associated with the "isochron" obtained cannot be adequately evaluated (fig. 19). These rocks, like the other gneiss domes, are biotitemuscovite gneisses with foliation concordant with both the intrusive margins and the schistosity in the adjacent andesitic assemblage rocks. The Harisi dome, like the Wadi Bagarah and Jabal Mina gneiss domes, intrudes and deforms rocks assigned to the Ablah Group (Anderson, 1978a). The apparent age (773 m.y.) and $r_{i}(0.7027)$ obtained from the two samples are consistent with results from the other two intrusives.

Rubidium-strontium studies of the garnet-bearing granitic gneiss of Wadi Bishah (loc. 4) indicate an apparent age of $664 \pm 9 \mathrm{~m} . y$., significantly less than the 761-m.y. average of the other four domes. The Index value of 1.01 is higher but cannot be interpreted as indicating disturbance of the $\mathrm{Rb}-\mathrm{Sr}$ system (fig. 19). The $r_{i}$ of $0.7035 \pm 0.0007$ is in agreement

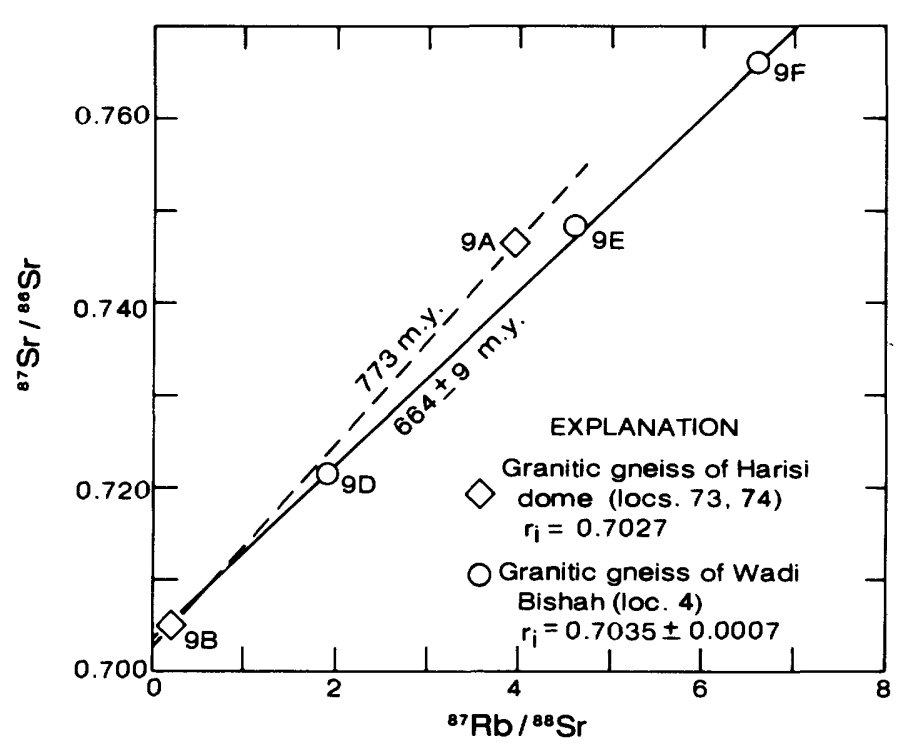

FIgURE 19.-Total-rock $\mathrm{Rb}-\mathrm{Sr}$ isochron diagram of the granitic gneisses of Harisi dome and Wadi Bishah. See figure 17 for explanation of isochron data. 
with values obtained for the other domes. The three sample sites within the body were separated by at least $100 \mathrm{~m}$ but represent the same general location. Because the body is strongly deformed and contains a metamorphic mineral assemblage, the possibility of metamorphic homogenization of strontium cannot be entirely disregarded. Amphibolites of the andesite unit that were intruded by the gneiss dome have high strontium concentrations and low ${ }^{87} \mathrm{Sr} /{ }^{86} \mathrm{Sr}$ ratios. Because of the relatively low strontium (95 $\mathrm{ppm}$, average) in the gneiss, exchange between the gneiss and amphibolites during metamorphism could reset the ${ }^{87} \mathrm{Sr} /{ }^{86} \mathrm{Sr}$ ratio to the low value of 0.7035 , even though the body has a $\mathrm{Rb} / \mathrm{Sr}$ ratio of 1.5 . Although we consider this possibility unlikely and regard the 664-m.y. age as a reasonable value for the age of intrusion, the data do not permit a more definitive conclusion. Regardless of whether the apparent age represents the time of intrusion or of metamorphism, major deformation occurred in the southern part of the Arabian Shield at or subsequent to $664 \pm 9$ m.y. ago.

\section{LATE-OROGENIC OR POSTOROGENIC GRANODIORITE TO GRANITE PLUTONS}

Fleck and others (1976), reporting K-Ar and ${ }^{40} \mathrm{Ar} /{ }^{39} \mathrm{Ar}$ ages for late-orogenic or postorogenic (socalled "Pan-African") granodiorite to granite plutons (fig. 3), conclude that most of the plutons were emplaced between about 610 m.y. and about 560 m.y., as determined by concordant mineral ages. Recalculating these data to the decay constants of Beckinsale and Gale (1969) and potassium abundance values of Garner and others (1975) changes this range to about $620 \mathrm{~m} . \mathrm{y}$. to $570 \mathrm{~m} . \mathrm{y}$. Rb-Sr results for 14 different granodiorite-granite plutons or intrusive complexes are shown in table 2. Totalrock isochron ages obtained for 8 bodies range from 587 to 643 m.y., but the youngest of these, from Wadi al Miyah (locs. 45, 46), has a very large uncertainty (one standard deviation is 17 percent) and high $r_{i}$. The remaining isochron ages cluster between $620 \mathrm{~m} . \mathrm{y}$. and $643 \mathrm{~m} . \mathrm{y}$., averaging $629 \mathrm{~m} . \mathrm{y}$. with a mean $r_{i}$ of 0.7032 . Model ages obtained for five other bodies by assuming this initial ratio $(0.7032)$ range from 608 m.y. to 657 m.y., with a mean of 632 m.y., nearly identical with the mean value of isochron ages.

The $\mathrm{Rb}-\mathrm{Sr}$ ages indicate that the oldest $\mathrm{K}$-Ar ages within the granodiorite to granite bodies are between 15 and 45 m.y. too young. Because many of these bodies contain no amphiboles and $\mathrm{K}-\mathrm{Ar}$ ages reported are for micas, some of the differentials might be explained by slow cooling. As discussed by Fleck and others (1976, p. 20), however, a later thermal pulse or phase between about 550 and 520 m.y. (recalculated values) is required by the irregular regional pattern of discordance in $\mathrm{K}$-Ar ages. Studies using ${ }^{40} \mathrm{Ar} /{ }^{39} \mathrm{Ar}$ ages indicate that even the oldest apparent ages have been affected to some degree. As shown by the Rb-Sr ages, the timing of the last major intrusive phase of shield formation was earlier than that defined by $\mathrm{K}-\mathrm{Ar}$ ages, commencing about 645 m.y. and continuing for at least 45 m.y., and probably longer.

\section{TINDAHAH BATHOLITH}

The Tindahah batholith of Coleman (1973a), in the eastern Khamis Mushayt quadrangle, intrudes deformed units of the andesite assemblage and gneissic to migmatitic bodies of quartz diorite to granodiorite, called the Khamis Mushayt Gneiss by Coleman (1973a), which represent the western part of the Wadi Tarib batholith. The Tindahah batholith, which ranges in composition from granodiorite to quartz monzonite, exhibits little or no evidence of deformation and forms an elongate body whose largest dimension parallels the fold axes and foliation in adjacent units. In contrast to many other late-orogenic or postorogenic bodies, the margins of the Tindahah batholith are irregular, invading adjacent stratified units both across and along structural trends. Rubidium-strontium total-rock data for the Tindahah batholith, shown in figure 20 , form an excellent isochron, with an age of $626 \pm 17$ m.y., $r_{i}$ of $0.7037 \pm 0.0004$, and Index of 0.16 . Potassium-argon ages reported by Fleck and others (1976) for this body range from 567 to 579 m.y. when recalculated using the new constants. Although the differences between K-Ar ages are not significant at the 95-percent level of confidence, they are clearly discordant with the $\mathrm{Rb}-\mathrm{Sr}$ age and indicate either slow cooling or subsequent reheating. As discussed by Fleck and others (1976), however, discordant ${ }^{40} \mathrm{Ar} /{ }^{39} \mathrm{Ar}$ age-spectra and the irregular regional pattern of $\mathrm{K}-\mathrm{Ar}$ ages indicate argon loss due to a thermal event subsequent to initial cooling.

\section{BANI THUWR PLUTON}

The Bani Thuwr pluton of Coleman (1973b) is a complex of coalescing ring structures that intrudes the same rock units as the Tindahah batholith approximately $20 \mathrm{~km}$ northwest of that body. It is a coarse-grained quartz monzonite containing large phenocrysts of pink microcline in some facies. Potassium-argon ages reported by Fleck and others 


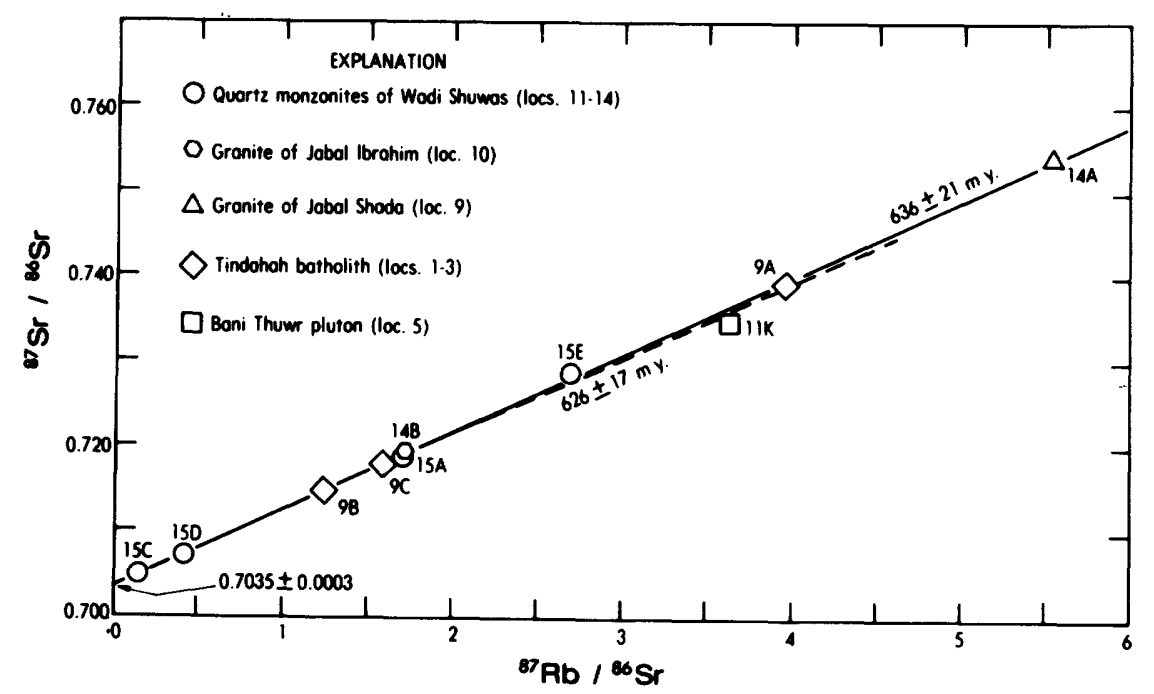

FIgURE 20.-Total-rock Rb-Sr isochron diagram of the Tindahah batholith and the Bani Thuwr pluton, quartz monzonites of Wadi Shuwas, and the granites of Jabal Shada and Jabal Ibrahim. Data for all isochrons are shown in table 2. Sample locations are shown in figure 4 and listed in table 1.

(1976) range from 529 m.y. to 590 m.y., with obvious discordance. Because only a single sample was suitable for Rb-Sr study, no isochron could be obtained. As shown in figure 20, the single data point falls somewhat below the isochron for the Tindahah batholith but is not significantly different at the 95-percent level of confidence. A model age of 608 m.y. is obtained by assuming an $r_{i}$ of 0.7032 , but its uncertainty could be as much as 30 m.y., because of analytical errors or an incorrect assumption of $r_{i}$.

\section{WADI SHUWAS}

Coalescing arcuate lenses or sheets of quartz monzonite, granodiorite, and quartz-bearing syenite (Greenwood, 1975a, 1975b) invade a dominantly diorite to quartz diorite terrane in the southeastern part of the Aqiq quadrangle and northeastern part of the Biljurshi quadrangle along Wadi Shuwas (locs. 11-14). Data from all of these units, called the quartz monzonites of Wadi Shuwas, form an isochron (fig. 20) with an age of $636 \pm 21$ m.y., $r_{i}$ of $0.7035 \pm 0.0003$, and Index of 0.43 , supporting the postulate of cogenesis. Potassium-argon ages reported by Fleck and others (1976) are discordant, yielding apparent ages from 618 m.y. to 545 m.y. and having hornblende values greater than those of coexisting biotite in all but one sample (71-8-15D, loc. 13). This single hornblende concentrate was examined by ${ }^{40} \mathrm{Ar} /{ }^{39} \mathrm{Ar}$ incremental-heating (Fleck and others, 1976), evolving a discordant age-spectrum with minimum age of 588 m.y. We conclude that the discordance cannot be due to slow cooling but rather is the result of argon loss subsequent to the 636-m.y. time of intrusion. The age of the event causing argon loss is poorly defined but is presumed to be less than 545 m.y. old.

\section{JABAL SHADA AND JABAL IBRAHIM}

Two small subcircular quartz monzonite to granite plutons occur in quadrangles adjacent to the Wadi Shuwas bodies at Jabal Shada (loc. 9) and Jabal Ibrahim (loc. 10). The single analyses reported (table 1) for each pluton represent the only samples from these locations, and sampling was made originally for K-Ar studies (Fleck and others, 1976). Recalculated to new constants, the K-Ar ages are from Jabal Shada, biotite 602 m.y. and muscovite 598 m.y. and for biotite from Jabal Ibrahim, 610 m.y. Rubidium-strontium model ages, shown in table 2, were calculated for these bodies assuming the mean $r_{i}$ of total-rock isochrons of the granodiorite to granite plutons (0.7032). Compared with the isochron for the nearby Wadi Shuwas bodies $\left(r_{i}=0.7035\right)$, however, the value for the Jabal Shada sample fits precisely and the Jabal Ibrahim data point is only slightly above (fig. 20). We conclude that the granite of Jabal Shada may have been cogenetic with the quartz monzonites of Wadi Shuwas (at 636 m.y.) and that the Jabal Ibrahim body may have been emplaced slightly earlier, between 640 and 657 m.y. ago.

JABAL QAL AND JABAL 'AYA

Quartz monzonite to granite plutons in the western part of the Jabal 'Aya quadrangle (locs. 21, 22) at Jabal Qal and Jabal Barkouk intrude metasedi- 
mentary and metavolcanic strata of both the basaltic and andesitic assemblages. Major north-south shear zones are truncated by the intrusives, and foliation in the metamorphic units adjacent to the bodies is deflected from the regional trend. The plutons are nearly circular bodies with arcuate screens of mafic rocks subparallel to the margins. They are undeformed and clearly postdate all major deformation within the area. As shown in figure 21 and table 2, sample values from these units form a linear array on the isochron diagram, yielding an age of $620 \pm 18$ m.y., $r_{i}$ of $0.7034 \pm 0.0005$, and Index of 0.03 . Data from a sample of a small closely related pluton at Jabal 'Aya (loc. 20) $15 \mathrm{~km}$ east of the Jabal Qal body also fit the isochron, suggesting that the Jabal 'Aya intrusive was coeval with Jabal Qal. Adding the data for Jabal 'Aya would reduce the isochron age slightly, yielding $614 \pm 13$ m.y., an $r_{i}$ of 0.7035 \pm 0.0004 , and an Index of 0.34 . The $614 \pm 13$-m.y. value represents a minimum age for the last movements on major north-trending shear zones in this area, which extend for distances as great as $300 \mathrm{~km}$ (see, for example, Greenwood and others, 1974).

\section{WADI HALAL}

The granodiorite of Wadi Halal intrudes a sequence of deformed ash-flow tuffs of the andesitic sequence in the Mayza quadrangle (Anderson, 1978b). The body is essentially circular but is itself cut by a younger reddish granitic phase. Foliations in the andesitic unit are deflected around the intrusion,

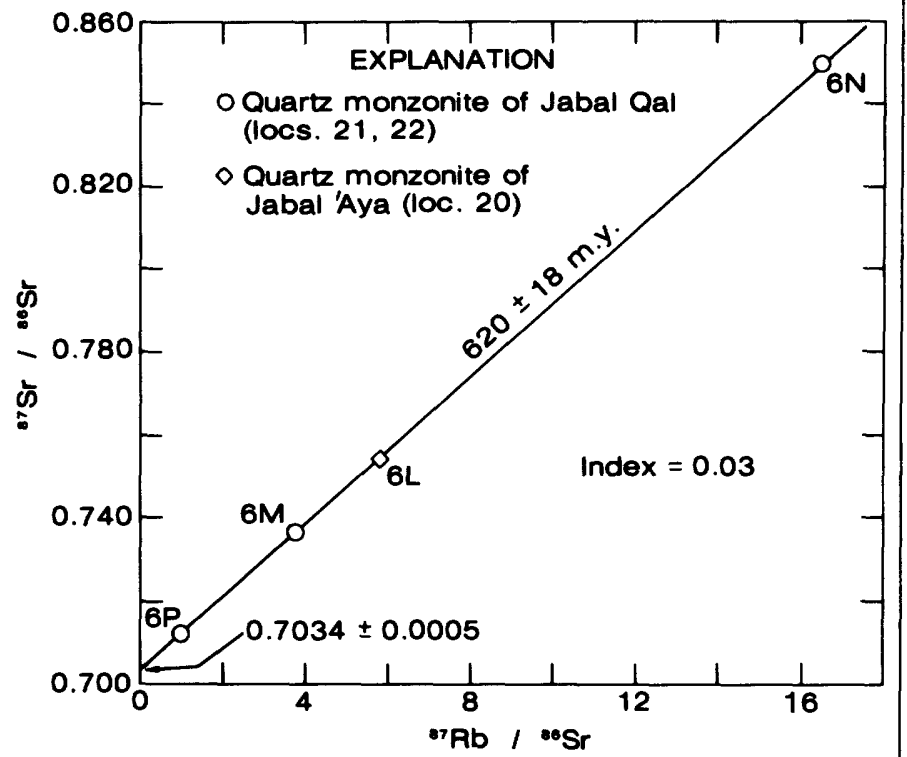

FIGURE 21.-Total-rock Rb-Sr isochron diagram of the quartz monzonites of Jabal Qal and Jabal 'Aya. See figure 20 for explanation of isochron data. which has metamorphosed some of these strata to the amphibolite facies. The metavolcanic rocks of Wadi Shuklalah (locs. 32, 33) are from this metamorphosed sequence, and as stated, yield a wholerock isochron age of $593 \pm 53$ m.y. Values for samples of the granodiorite are plotted in figure 22, where the array defines a good isochron showing an age of $643 \pm 20$ m.y., $r_{i}$ of $0.7028 \pm 0.0002$, and Index of 0.52. Samples 742-20A and 742-20B were collected approximately $0.5 \mathrm{~km}$ east of the other samples in what Anderson maps as a separate "border" phase of the body. Both samples appear to be displaced slightly above the isochron, indicating a slightly higher $r_{i}(0.7031)$ but an identical (2-point isochron) age of 644 m.y. The younger "red-granite" phase that intrudes the granodiorite may well be responsible for homogenization of strontium isotopes in the metavolcanic rocks at about 593 m.y. That phase was not collected as part of the current study, however. Because much of the granodiorite of Wadi Halal is foliated, it is hypothesized that emplacement may have been diapiric, as the magma of the younger, granitic phase drove a nearly circular "piston" of metavolcanic rocks and granodiorite upward. This hypothesis is strengthened by the presence of subcircular fractures that are generally concentric with arcuate sheets of red granite.

\section{WADI AL MIYAH AND WADI MUSAYRAH}

Granodiorite units in the area north and east of the town of Bishah (fig. 3) show a vague, possibly primary, foliation but are structurally, chemically,

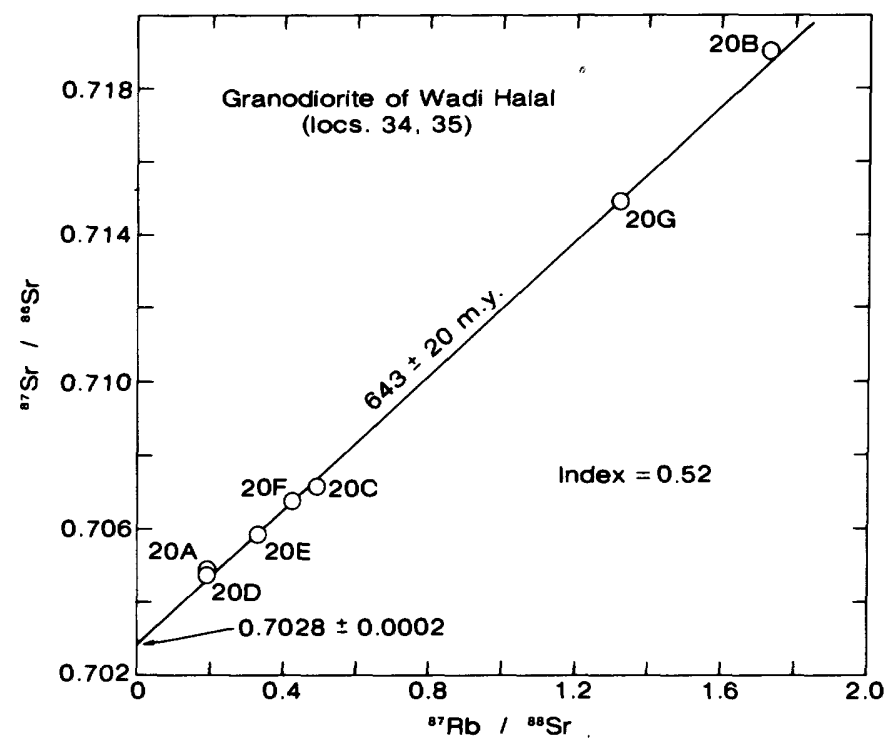

FIGURE 22.-Total-rock $\mathrm{Rb}-\mathrm{Sr}$ isochron diagram of the granodiorite of Wadi Halal. See figure 20 for explanation of isochron data. 
and isotopically distinct from the highly deformed (gneissic) granodiorite and quartz diorite bodies such as those of the southwestern part of the $\mathrm{Al}$ Qarah quadrangle (locs. 51, 87) that give ages of $724 \mathrm{~m} . \mathrm{y}$. Samples of the granodiorite of Wadi Musayrah (locs. 48-50, fig. 4) define a precise isochron (fig. 23) of $623 \pm 18 \mathrm{m.y}$. with an $r_{i}$ of $0.7033 \pm 0.0003$ and Index of 0.42 . The average $\mathrm{Rb} / \mathrm{Sr}$ ratio of the samples is significantly greater than those of the older batholiths (table 2), while Sr concentrations are less than half (table 1). The granodiorite of Wadi al Miyah (locs. 45, 46) intrudes metavolcanic strata of the andesite unit (loc. 47), but its relation to dioritic units in the region is not known. Feldspars in samples of the Wadi al Miyah body are somewhat altered, but it is not clear whether this alteration accompanied deformation or was significantly later. A statistically acceptable isochron may be drawn through the three data points, giving an apparent age of $587 \pm 77$ m.y. (fig. 23), but the large uncertainty reflects both the dispersion of the data and the inadequate spread in $\mathrm{Rb} / \mathrm{Sr}$ ratio. Only one point from the Wadi al Miyah set, 742-25L, diverges significantly from the Wadi Musayrah isochron. That point has the highest $\mathrm{Rb} / \mathrm{Sr}$ ratio and falls below the isochron, a characteristic pattern of geologic error in $\mathrm{Rb}-\mathrm{Sr}$ systems (see, for example, Brooks and others, 1972). The data are consistent with a $623-\mathrm{m} . y$. age for both bodies, although stron-

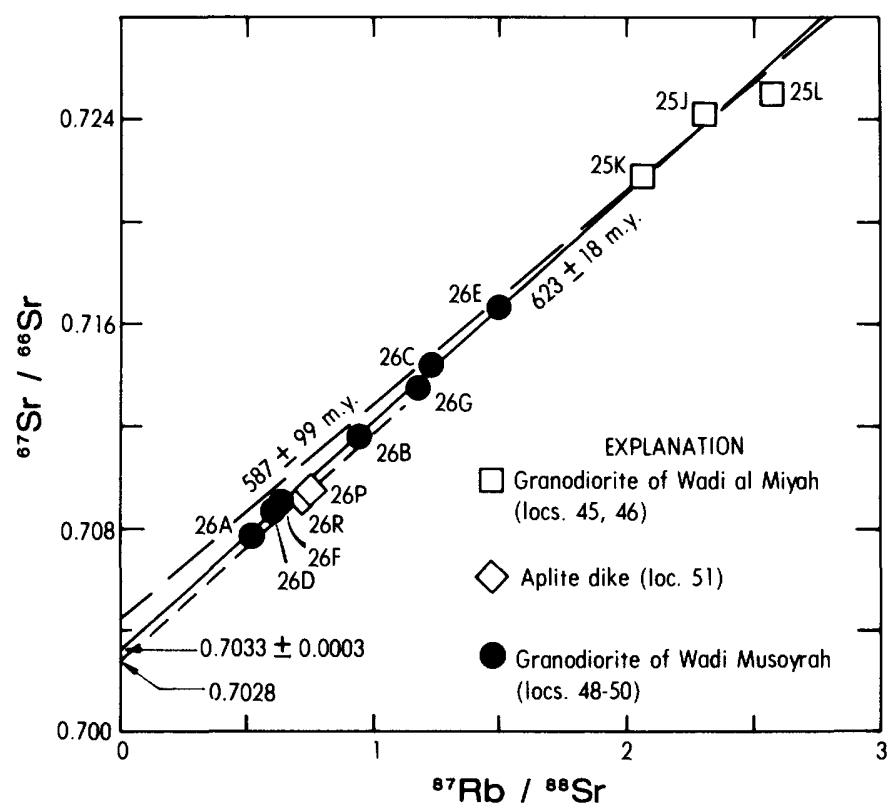

FIGURE 23.-Total-rock Rb-Sr isochron diagram of the granodiorites of Wadi al Miyah and Wadi Musayrah and an aplite dike of the southwestern Al Qarah quadrangle. See figure 20 for explanation of isochron data. tium concentrations in the Wadi al Miyah samples are much lower (table 1).

On its southern and eastern margins, the granodiorite of Wadi Musayrah intrudes a broad area of quartz diorite discussed previously (locs. 17-19, 51, 87 ; fig. 15) that gives an age of 723 m.y. At one of these localities (loc. 51) an aplite dike was sampled that cuts across foliation and compositional banding in the gneissic quartz diorite but is tightly folded coaxially with isoclinal folds in the banding of the gneiss. As the dike is less tightly folded than is the banding in the quartz diorite, its emplacement clearly postdated initial deformation. The folding, however, does indicate significant deformation subsequent to intrusion, probably at temperatures sufficiently elevated for plastic flow. $\mathrm{Rb}-\mathrm{Sr}$ data for two samples of the dike are shown in figure 23. The points fall slightly below the isochron of samples of the granodiorite of Wadi Musayrah located 15 to $20 \mathrm{~km}$ to the north, but a line through the two points is clearly parallel to the isochron. The apparent age of the two-point "isochron" is $621 \mathrm{~m} . y$. ., essentially identical with the Wadi Musayrah value of $623 \pm 18$ m.y. The $r_{i}$ of the dike, 0.7028 , is equal to the lowest value obtained from any of the late-orogenic or postorogenic bodies but is exactly the value to be expected if the aplite were derived by partial melting (mobilization) of the 723-m.y.-old gneissic host rock at that location (southwestern Al Qarah quadrangle) $623 \mathrm{m.y}$. ago.

The weak foliation of the granodiorites of Wadi Musayrah and Wadi al Miyah and the obvious deformation of the aplite dike of the same age show that deformation and, undoubtedly, metamorphism continued in the Bishah area after 620 m.y. ago. Because this deformation involved folding and the development of foliation along the major north-south trend at a time when all similar deformation in the area of Jabal Qal (loc. 21, 22) had ceased, deformation in the Bishah area must have occurred, or at least continued, after that to the west.

Although the granodiorites described are among the youngest intrusive bodies in the shield, a still younger "red granite" represents the youngest intrusive unit within this region. Potassium-argon studies by Fleck and others (1976) indicate concordant biotite and hornblende ages of about 605 m.y. from these red granites, although some biotite and plagioclase ages are as young as $536 \mathrm{~m} . \mathrm{y}$ and indicate subsequent loss of radiogenic argon. The red granites intrude the granodiorite of Wadi al Miyah, apparently disturbing the Sr isotopic systems to some degree. Because of the low strontium 
concentrations and high $\mathrm{Rb} / \mathrm{Sr}$ ratios of the Wadi al Miyah granodiorite, small disturbances of the system could be apparent even though they may occur within $30-50$ m.y. after intrusion.

\section{OTHER LATE-OROGENIC OR POSTOROGENIC PLUTONS}

Late-orogenic or postorogenic intrusions in the northeastern part of the region studied (fig. 3) show the same range in age as those in the southern and western parts, but in the northeast the bodies are generally deformed by the Najd fault system (see, for example, Greenwood and Brown, 1973; Hadley, 1976). The system is represented not only by northwest-trending, apparently left-lateral strike-slip faults but also by penetrative deformation of units adjacent to the major shear zones, forming strong planar and linear fabrics. These structures, however, have distinctly northwesterly trends, whereas the generally older regional trends are more nearly north-south or slightly northeasterly.

Effects of deformation after 600 m.y. ago are well displayed in the Bi'r Juqjuq quadrangle (locs. 52-61), studied by Hadley (1976). Although the resetting of $\mathrm{Rb}-\mathrm{Sr}$ ages of units of the andesitic assemblage can probably be related to emplacement of the granodiorite to granite plutons, the age of hornblende diorite in the eastern part of the quadrangle (loc. 58) cannot. As seen on the isochron diagram for samples of this unit, figure 24, the three samples, all with $\mathrm{Rb} / \mathrm{Sr}$ ratios less than 0.03 , yield an apparent age of $522 \pm 429$ m.y., $r_{i}$ of $0.7033 \pm 0.0003$, and Index of 0.07 . The samples all show petrographic evidence of greenschist-facies metamorphism, and the unit is intruded by a granodiorite to quartz monzonite complex that occurs within much

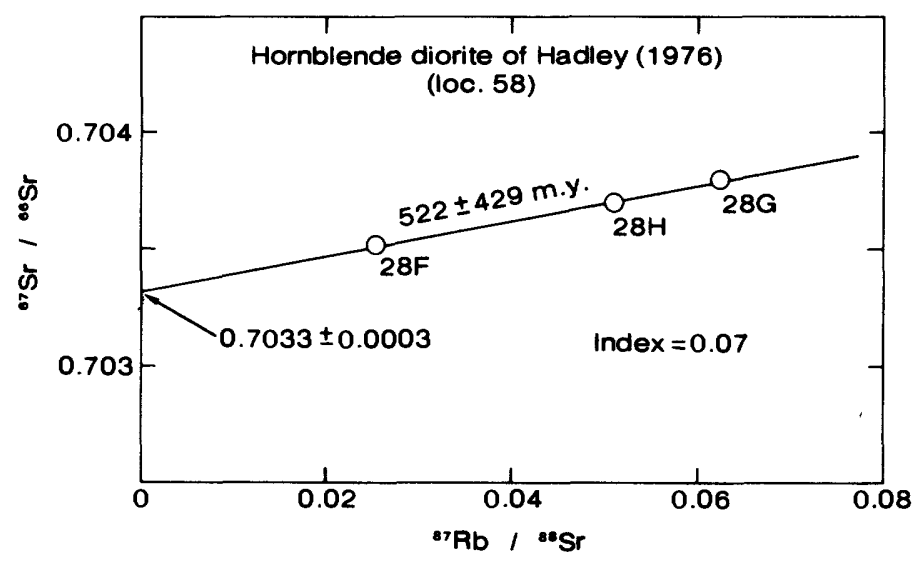

FIGURE 24.-Total-rock $\mathrm{Rb}-\mathrm{Sr}$ isochron diagram of the hornblende diorite of Hadley (1976). See figure 20 for explanation of isochron data. of the eastern one-third of the Bi'r Juqjuq quadrangle. Of the $\mathrm{Rb}-\mathrm{Sr}$ results from samples from four different locations (locs. 56, 57, 59, 61) within this complex intrusion, shown in the isochron diagram of figure 25, only the two from the Jabal Tarban area (loc. 61) have sufficient variation in $\mathrm{Rb} / \mathrm{Sr}$ ratio to give an independent indication of age for a single locality. That value is $635 \mathrm{~m} . \mathrm{y}$., with an intercept of 0.7031 , close to the average $r_{i}(0.7032)$ of isochrons from rocks of this group. The remaining granodiorite or quartz monzonite samples show significant dispersion that may be the result of metamorphism but also may indicate differences in age and (or) $r_{i}$ within the intrusive complex. Model ages generated by using the weighted mean ${ }^{87} \mathrm{Sr} /{ }^{86} \mathrm{Sr}$ and ${ }^{87} \mathrm{Rb} /{ }^{86} \mathrm{Sr}$ values for each area and and assuming an $r_{i}$ of 0.7032 yield apparent ages of 620 m.y. for the gneissic granodiorite (loc. 56, 57) and 636 m.y. for quartz monzonite to the northeast (loc. 59). If all samples are presumed to be coeval, they yield an isochron age of $651 \pm 25 \mathrm{~m} . y ., r_{i}$ of $0.7029 \pm 0.0004$, and Index of 0.92 . These values indicate a significantly greater age than the age of the hornblende diorite (522 m.y.) intruded by the complex. In addition to showing evidence of greenschist-facies metamorphism, the hornblende diorite body is cut by en echelon fractures of the Najd fault system, is the host for a closely spaced system of mafic dikes that parallel the trend of the Najd system, and contains a pervasive foliation with a similar northwest trend. We suggest that the apparent age of the diorite may represent the time of homogenization of strontium as a result of deformation within the Najd fault system, although the uncertainty in the age is ex-

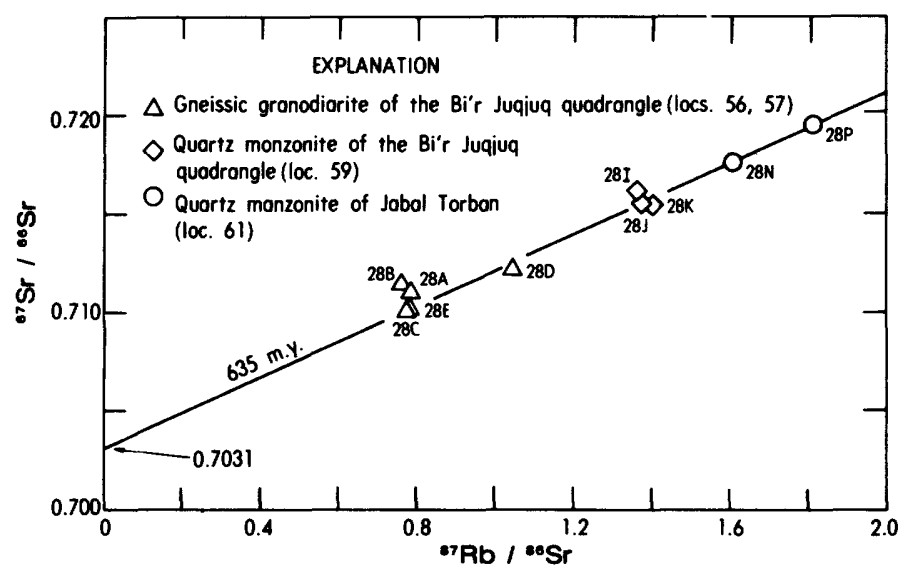

FiguRe 25.-Total-rock $\mathrm{Rb}-\mathrm{Sr}$ isochron diagram of the quartz monzonite and gneissic granodiorite of the Bi'r Juqjuq quadrangle and the quartz monzonite of Jabal Tarban. See figure 20 for explanation of isochron data. 
ceptionally large because of the small range of the $\mathrm{Rb} / \mathrm{Sr}$ ratio. By this interpretation, the 522-m.y. value would reflect the closing of strontium isotopic systems at the end of major deformation and greenschist metamorphism along the Najd fault system and is quite similar to that suggested by Fleck and others (1976) for that event.

\section{DISGUSSION OF THE RESULTS}

Rubidium-strontium ages reported here represent all major rock units of the southern part of the Arabian Shield with the exception of the layered gabbros and several exposures of serpentinite. The layered bodies are considered to be largely late-orogenic or postorogenic but older than most of the granodiorite to granite plutons (Coleman and others, 1972). In the Khaybar quadrangle (Coleman, 1973b), hornblende from the Jabal Shayi gabbro (Coleman and others, 1973) yields an ${ }^{40} \mathrm{Ar} /{ }^{39} \mathrm{Ar}$ age of $626 \pm 8 \mathrm{~m} . \mathrm{y}$. (corrected to new decay constants) and is intruded by the Bani Thuwr pluton (608 m.y., table 2). The gabbro postdates the granitic gneiss of Wadi Bishah (loc. 4), which yields an age of $664 \pm 9$ m.y. (table 2 ). These data and those for other gabbros reported by Fleck and others (1976) are consistent with an age range of 620-660 m.y. for the layered gabbros. With the exception of the serpentinite, then, the $\mathrm{Rb}-\mathrm{Sr}$ data reported here provide a detailed chronology for the evolution of the shield.

The Arabian Shield is not Archean continental crust remobilized during one or more periods of intracratonic events. There is at this time no evidence to support the presence of any sialic crust before 1,000 m.y. ago. The oldest plutonic rocks studied are about $900 \mathrm{~m} . \mathrm{y}$. old. Units of the basaltic assemblage represent the oldest rocks in the southern part of the shield and the constituent rock types indicate marine sedimentation and volcanism remote or isolated from a continental landmass. Whether part of the andesitic assemblage might be as old as the 1,165-m.y. age obtained from the basaltic assemblage cannot be resolved at this time. The oldest rocks of the andesitic assemblage studied yield an age of $912 \pm 76 \mathrm{~m} . \mathrm{y}$., almost precisely the age of the oldest plutonic rocks studied.

Although none of the major rock units has been so adequately sampled as to represent the entire shield, the similar age ranges, initial ${ }^{87} \mathrm{Sr} /{ }^{86} \mathrm{Sr}$ ratios, chemical compositions and $\mathrm{Rb} / \mathrm{Sr}$ ratios of flow units of the andesitic assemblage and the dioritic batholiths are strong evidence of a common genesis. The greatest time spans not represented by either andesitic or dioritic units during the period from 920 to 720 m.y. are 37 m.y. (between 890 and 853 m.y.) and 30 m.y. (between 815 and 785 m.y.). Considering the problem of representative sampling of an area the size of the Arabian Shield, as well as the uncertainties in the ages themselves, however, these "gaps" cannot be assigned much significance at present.

The model of Proterozoic evolution of the Arabian Shield presented by us previously (Greenwood and others, 1976) is consistent with data presented here, although the earlier results have been modified to reflect the newly recommended decay constant for ${ }^{8} \mathrm{Rb}$ as well as the analyses of additional samples. Although the tectonic episodes delineated by Greenwood and others (1976), which represent specific deformational periods in the areas where they were defined, may eventually be recognized more widely, we cannot extend these "events" shieldwide. At given periods of time, deformation, uplift, and erosion occurred in some areas while others were the sites of rapid accumulation of volcanic, volcaniclastic, and even carbonate strata. This situation was reversed later, providing evidence of repeated local cycles of deposition, deformation, intrusion, and erosion. Folded schistosities in the oldest strata demonstrate the repetitive nature of deformation, although folds from later events may be coaxial with those developed earlier. In short, multiple tectonic and magmatic phases are detectable within specific areas during the period between 920 and $680 \mathrm{~m}$.y. ago, but some of these episodes were both temporally and areally restricted and did not affect broad areas of the Arabian Shield. This period of andesitic volcanism, diorite intrusion, island-arc sedimentation, and deformation with generally north-south structural-trends is temporally defined by the ages of the diorite batholiths. We favor treatment of this orogenesis as a single geologic event, the Hijaz tectonic cycle or orogeny (Brown, 1972; Greenwood and others, 1973, 1976), which represents the major period of development of the Arabian subcontinent and formation of continental crust within an "oceanic" geologic environment.

Whereas plutonism and island-arc volcanism and sedimentation of the Hijaz tectonic cycle spanned a period of about $200 \mathrm{~m} . \mathrm{y}$., the culminating plutonic phase of orognesis was comparatively brief, covering approximately $40 \mathrm{~m} . \mathrm{y}$. between about 650 and 610 m.y. ago. Magmas of this late-orogenic phase were much more evolved than those in earlier phases; plutons average over 70 percent $\mathrm{SiO}_{2}$, 
whereas diorite bodies, including trondhjemite, average only 64 percent (Greenwood and Brown, 1973). As shown by Greenwood and Brown (1973), the differentiation trends of the two groups diverge significantly, suggesting derivation of the magmas from different source materials.

Rubidium-strontium studies not only support the interpretation of differences in the sources of Hijaz and Pan-African magmas but also provide data that reflect the evolution of sialic crust in Saudi Arabia. From correlation diagrams of $\left({ }^{87} \mathrm{Sr} /{ }^{86} \mathrm{Sr}\right)_{0}$ and $(\mathrm{Rb} / \mathrm{Sr}$ ) with age (fig. 26), it is evident that both ratios are universally low in the early magmas. Even the metavolcanic rocks of Wadi bin Dwaynah (912 m.y.), which contain 74 percent $\mathrm{SiO}_{2}$, have low $\mathrm{Rb} / \mathrm{Sr}$ ratios and probably represent trondhjemitic magma. Between 770 and 780 m.y., however, magmas enriched in rubidium $(\mathrm{Rb} / \mathrm{Sr}$ ratios greater than 0.3) appeared for the first time. Magmas in which $\mathrm{Rb} / \mathrm{Sr}$ ratios were below 0.2 were still common, but the average value was distinctly greater
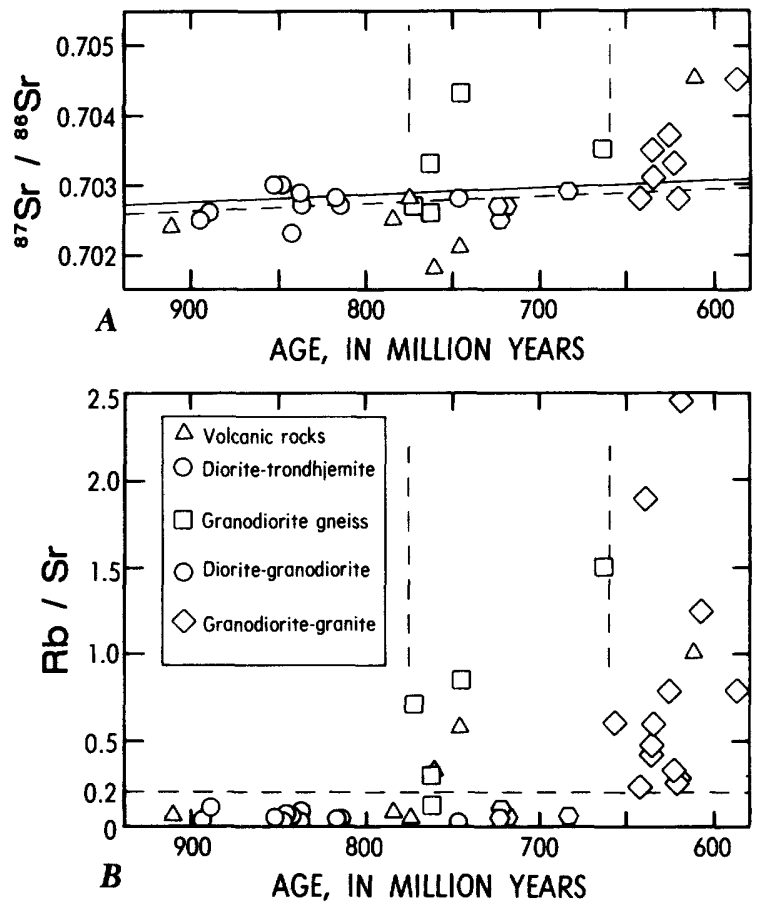

FIgURE 26.--Variation of $(A)$ initial ${ }^{87} \mathrm{Sr} /{ }^{88} \mathrm{Sr}$ ratio and $(B)$ $\mathrm{Rb} / \mathrm{Sr}$ with age. Vertical dashed lines are drawn at two periods of major change in strontium isotope composition and $\mathrm{Rb} / \mathrm{Sr}$ ratio of magmas. Strontium-evolution line (solid line) in $A$ represents single-stage evolution from a meteorite initial ${ }^{87} \mathrm{Sr} /{ }^{88} \mathrm{Sr}$ ratio of 0.699 to the average modern island-arc value of 0.7037 (Faure and Powell, 1972; Dickinson, 1970). Dashed evolution line is least-squares fit of data points shown here and listed in table 2 having $\mathrm{Rb} / \mathrm{Sr}$ ratio less than 0.2 , yielding ${ }^{87} \mathrm{Sr} /{ }^{86} \mathrm{Sr}$ values of 0.7036 for the present and 0.6989 at 4,500 m.y. than that of the more primitive diorite-trondhjemite suite. Although many of the plutons having $\mathrm{Rb} / \mathrm{Sr}$ ratios greater than 0.2 occur as gneiss domes, volcanic units are also represented in this group. We suggest that the change in magma type reflects an evolution of the source from one dominated by oceanic lithosphere and perhaps mantle to one including previously differentiated (that is, sialic) crust. Because this sialic component is juvenile, however, no increase in initial ${ }^{87} \mathrm{Sr} /{ }^{86} \mathrm{Sr}$ ratios is observed. As shown by Kistler and Peterman (1973), in places where magma was derived at least in part from ancient sialic crust ${ }^{87} \mathrm{Sr} /{ }^{86} \mathrm{Sr}$ ratios are elevated above those of magma derived solely from mantle or oceanic lithosphere.

Initial ${ }^{87} \mathrm{Sr} /{ }^{86} \mathrm{Sr}$ ratios obtained in this study (table 2) are compared to an average oceanic basalt strontium-evolution line (solid line, fig. 26A) representing a "single-stage" or constant $\mathrm{Rb} / \mathrm{Sr}$-ratio model for strontium evolution (Faure and Powell, 1972; Gast, 1967). The evolution line was constructed using an average ${ }^{87} \mathrm{Sr} /{ }^{86} \mathrm{Sr}$ ratio of 0.7037 for modern oceanic island-arc andesites and basalts (Faure and Powell, 1972 ; Dickinson, 1970; Peterman and Hedge, 1971) and an initial ${ }^{87} \mathrm{Sr} /{ }^{86} \mathrm{Sr}$ ratio $\left(r_{i}\right)$ of 0.699 and age of $4.5 \times 10^{9} \mathrm{yr}$ for the Earth. As shown by Peterman and Hedge (1971) and Brooks and others (1976), $r_{i}$ values of modern magmas show significant variation, much of which is probably related to source heterogeneities. Data presented in figure 26 show similar ranges, as would be expected. The evolution line is considered to be only an average and-depends critically upon the assumption of constant average $\mathrm{Rb} / \mathrm{Sr}$ ratio $(0.025)$ in the source region. The close agreement between this line and initial ratios of early shield-forming units from Saudi Arabia, however, is impressive (fig. 26). Not only does the best-fit strontiumevolution line for primitive Arabian magmas, as defined by data from all units studied having $\mathrm{Rb} / \mathrm{Sr}$ ratios less than 0.20 (dashed line, fig. 26A), demonstrate the agreement of initial ${ }^{87} \mathrm{Sr} /{ }^{86} \mathrm{Sr}$ with a linear island-arc strontium-evolution model, but also the slope of this line indicates a similar $\mathrm{Rb} / \mathrm{Sr}$ ratio for the source area (0.025). Extrapolated to $4.5 \times 10^{9}$ yrs, this line yields a ${ }^{87} \mathrm{Sr} /{ }^{86} \mathrm{Sr}$ ratio of 0.6989 , where its zero-age (modern) value would be 0.7036 . We conclude that the early arc-building magmas in Saudi Arabia were both compositionally and isotopically identical to those that would have been formed during the period from 900 to 780 m.y. ago in intraoceanic island arcs and were distinctly different from those that would have formed ensiali- 
cally from ancient continental crust. Magmas formed between 770 and 680 m.y. ago were more variable in ${ }^{8 \tau} \mathrm{Sr} /{ }^{86} \mathrm{Sr}$, but the values for these ratios nevertheless average near the evolution line. Because these average values are consistent with the model, no significant amounts of older sialic material could have been involved, although involvement of very juvenile "granitic crust" cannot be excluded.

Units studied that formed after 670 m.y. are significantly enriched in rubidium relative to older rocks, and their $\mathrm{Rb} / \mathrm{Sr}$ ratios are greater than 0.20 , without exception. As stated, however, no analyses were made of layered gabbro that formed between about 660 and 620 m.y. ago, but the presence of these mafic rocks suggests a bimodal distribution of the $\mathrm{Rb} / \mathrm{Sr}$ ratio during that period. Volumetrically, gabbro constitutes only a small proportion of the younger magmas, whereas almost all Pan-African magmas had compositions ranging from granodiorite to granite and $\mathrm{Rb} / \mathrm{Sr}$ ratios greater than 0.20 . Initial ${ }^{87} \mathrm{Sr} /{ }^{86} \mathrm{Sr}$ values for most of the Pan-African plutons fall somewhat above the best-fit strontiumevolution line of the older rocks (fig. 26), although in most cases the differences from the line are not significant at the 95-percent level of confidence. The slight elevation of the average initial ratio of these younger magmas above the strontium-evolution line, however, when combined with the marked increase in $\mathrm{Rb} / \mathrm{Sr}$ ratio, suggests a contribution to the melts from a more highly differentiated source than that from which earlier units were derived. Because the $r_{i}$ values of the older units of the area are inconsistent with the presence of ancient sialic crust, the only possible source of sialic material in Arabia would have been the early arcforming units themselves. Although still low, the average $\mathrm{Rb} / \mathrm{Sr}$ ratio of analyzed rocks older than $670 \mathrm{~m} . \mathrm{y}$. is 0.15 , well above the 0.025 value indicated for their source by their strontium-evolution line (fig. 26). Partial melting at 650-600 m.y. of this thickened, more siliceous, and higher $\mathrm{Rb} / \mathrm{Sr}$ lower crust formed between 900 and 700 m.y., could produce both the higher $\mathrm{Rb} / \mathrm{Sr}$ ratios and the slightly elevated $r_{i}$ values of the Pan-African plutons. The fact that the $r_{i}$ values of these plutons fall only slightly above the theoretical strontium-evolution line for oceanic island arcs indicates that this sialic component in the source of Pan-African magmas was juvenile, with insufficient time for significant enrichment in ${ }^{87} \mathrm{Sr}$.

When the age determinations of dioritic bodies (table 2) are plotted geographically (fig. 27), a general decrease in age is seen from west to east (or southwest to northeast) across the southern part of the shield, although the sparcity of data does not justify any attempt to delineate age provinces or gradients. As will be noted from the small variation in $\mathrm{Rb} / \mathrm{Sr}$ ratios of the diorite bodies, little if any systematic variation in rock type occurs, even though the ages have a range of almost 200 m.y. These data suggest an eastward migration of the axis of magmatism and presumably the volcanic arc itself with time. West of the major north-trending fault system that extends from north of Jizan toward Ranyah along Wadi Bishah at about long $42^{\circ} 30^{\prime} \mathrm{E}$, most dioritic complexes are older than 800 m.y. As shown in figure 26, only low $\mathrm{Rb} / \mathrm{Sr}$ magmas formed before about 775 m.y. ago, when granodiorite gneiss domes such as those at Jabal Mina and Wadi Bagarah were emplaced. These more highly differentiated bodies not only constitute the youngest plutonic units of the Hijaz cycle in each region but also suggest an evolution of the source of orogenic magmas. To the southeast, dioritic magmas range from 843 to 684 m.y., but only the oldest are associated with trondhjemite. East of Bishah, units representing the greatest volume of magma range from diorite to granodiorite with ages between 750 and 680 m.y. These data document an eastward or northeastward migration of magmatic activity with the earliest magmas in each area being dioritic, but evolving to granodiorite as the volume of sialic material in the source regions increased.

In contrast to the geographic distribution of plutonic rocks of the Hijaz cycle, that of late-orogenic or postorogenic granitic rocks is shieldwide (fig. 28). The migration of plutonic activity recorded by the decrease in ages of dioritic complexes from west to east does not continue in plutons younger than about 660 m.y. The youngest apparent age, $587 \pm 99$ m.y., granodiorite in the Wadi Miyah quadrangle, is from the north-central part of the area; but, as noted, this rock may have been affected by later alteration. Ages reported here (table 2) indicate a shieldwide period of largely postorogenic plutonism between $660 \mathrm{~m} . \mathrm{y}$. and about $610 \mathrm{~m} . \mathrm{y}$. with some indication of subsequent late-stage emplacement of red granite, some of which may be peralkaline.

Although the dioritic magmas are associated with the early evolution of an island arc, the granodiorite to granite suite differs from the diorites in age, composition, degree of deformation, and intrusive style, suggesting to us a genetic difference as well. This younger suite is contemporaneous with largely detrital sedimentary strata containing much less 


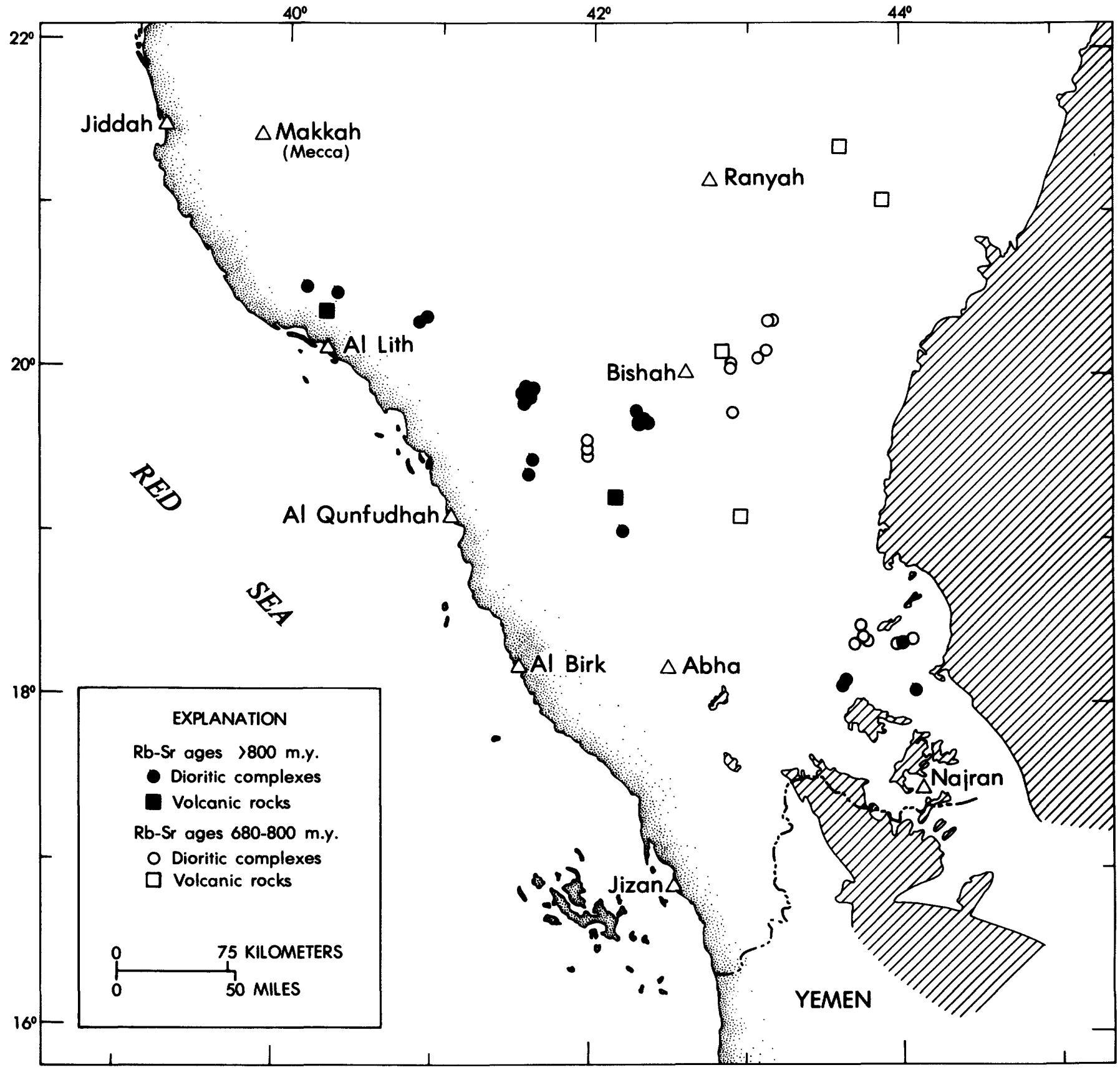

FIGURE 27.-Distribution of dioritic complexes and metavolcanic rocks older than 680 m.y.

primary volcanic material dominated by the products of presumably subaerial eruptions, including ash-flow tuff. Even though the older units of the andesitic assemblage were deposited in an area remote or isolated from eroding continental masses, by $\mathbf{5 4 0}$ m.y. ago the Arabian Shield had become part of the African plate, and the granodiorite to granite suite represents the last major magmatic event. We suggest that this event, known as the Pan-African event or orgeny (Kennedy, 1964; Holmes, 1965; Clifford, 1967, 1968, 1970) and expressed not only in Saudi Arabia but throughout much of the eastern part of the African continent, represents a phase of continental collision or suturing of Arabia to the Proterozoic African plate. Subduction would have ceased as the continental margin of Africa 


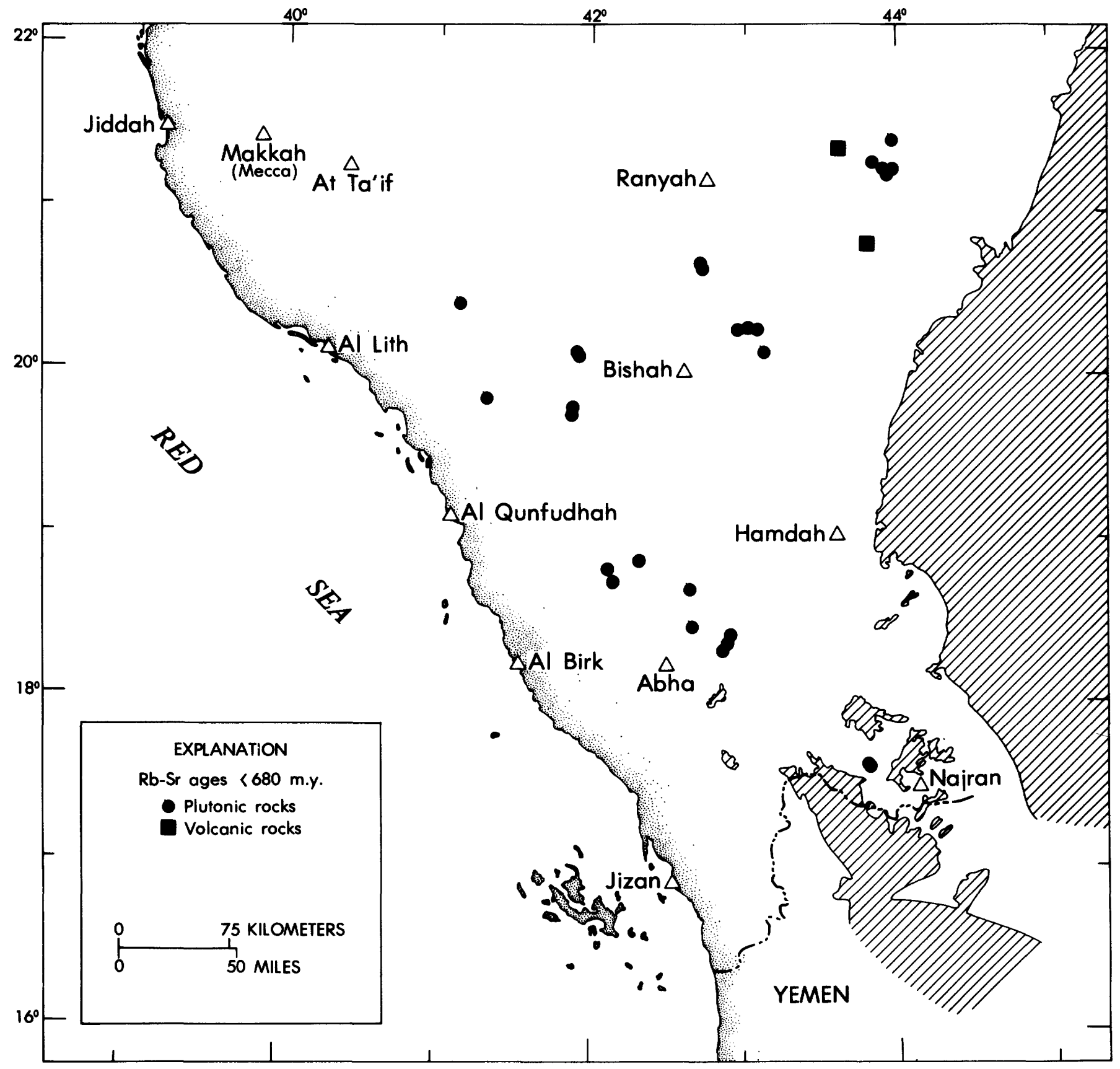

FIGURE 28.-Distribution of volcanic rocks and late-orogenic or postorogenic plutonic rocks less than $680 \mathrm{~m} . \mathrm{y}$. old.

entered the trench, making this event the culminating phase of a prolonged period of plate convergence.

Our model suggests that prior to the collisional phase, consumption of a margin of oceanic crust on the east side of the Proterozoic African plate by subduction along an eastward-dipping oceanicoceanic plate-boundary (island-arc-type subduction zone) generated new sialic crust along the Arabian island arc (fig. 29). As subduction proceeded, the distance between the Proterozoic African Continent and the Arabian island arc decreased, and progressively larger amounts of terrigenous detritus were added to andesitic volcanic and sedimentary sequences in the arc (see fig. 2). Because the areas of active volcanism, plutonism, and sedimentation mi- 
WEST
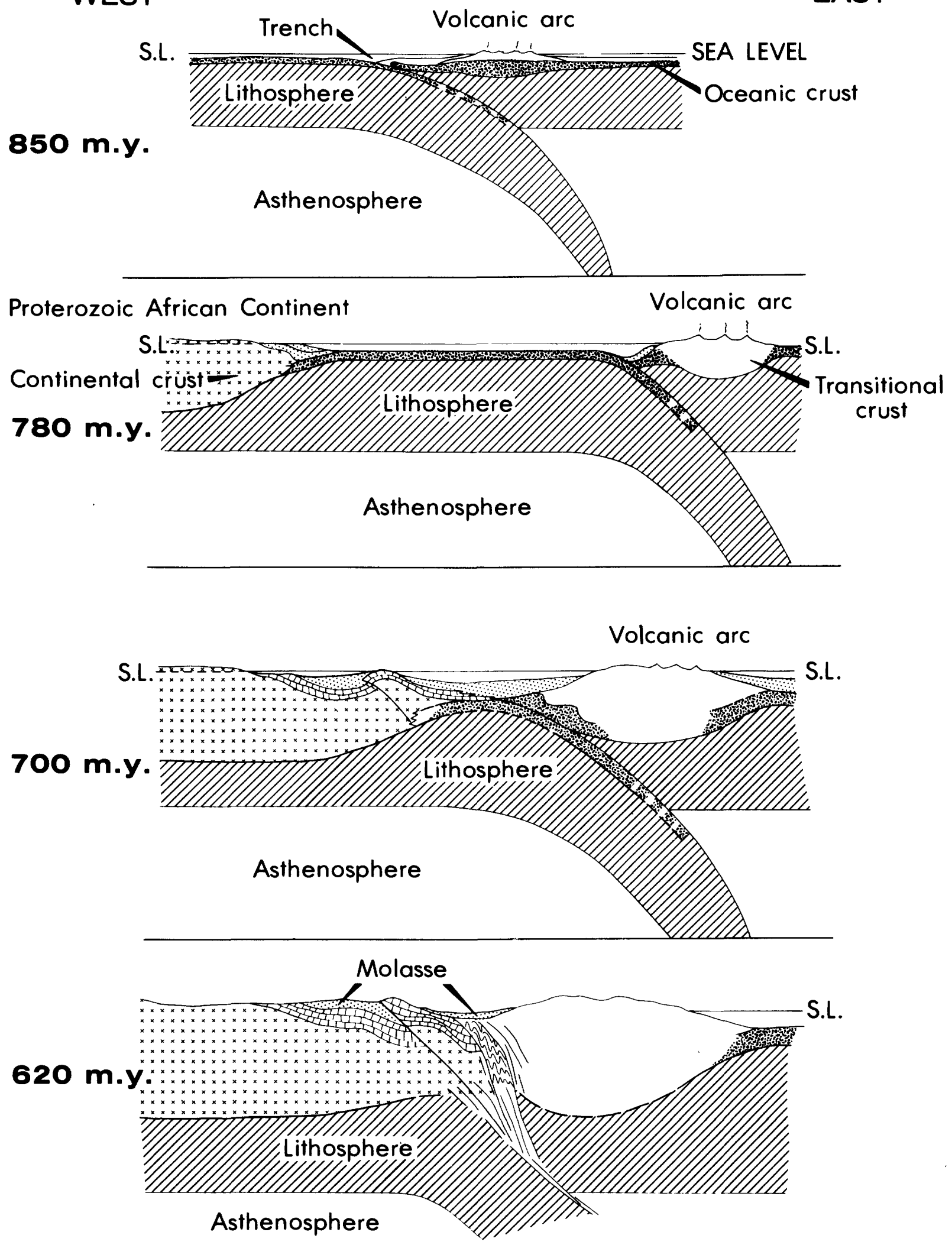

FIGURE 29.-Plate-tectonic model of the origin and deformation of the Arabian Shield. 
grated to the eastern part of the Arabian island arc before collision, however, exposure of the older parts of the are may have provided both a source of juvenile plutonic detritus and a barrier limiting amounts of African continental detritus. Because subduction would have continued until the time of actual collision of the African continent with the island arc and because the axis of subduction-related volcanism and plutonism (that is, andesite and diorite) was migrating eastward, the hiatus between island-arc volcanism and intrusion of the Hijaz cycle and the onset of collision-related (Pan-African) intrusion and tectonism would decrease from west to east. In the easternmost parts of the Arabian arc, subduction-related tectonism and plutonism would have progressed directly into those related to collision with no hiatus at all. Deformation of 640-630m.y.-old granodiorite in the Bi'r Juqjuq and Mayza quadrangles and the presence of 684-m.y.-old gneissic quartz diorite in Wadi Malahah may be evidence of this transition.

As collision began, deformation, metamorphism, and granodiorite to granite plutonism became shieldwide. The collisional phase (the Pan-African event) probably began prior to 650 m.y. ago, subsequent to emplacement of the major diorite batholiths. Location of a suture or sutures between the Proterozoic African plate and the island arc or arcs is currently uncertain, although the report of an ophiolite body at Jabal al Wask (Bakor and others, 1976) raises the possibility that part of such a structure may lie within the Arabian Peninsula. Study of both diorite and granodiorite to granite intrusive bodies in northeastern Sudan (Neary and others, 1976), however, revealed the presence of intrusive rock types characteristic of the Arabian Shield. Darracott (1972) reports a gravity "signature" of the edge of the Pan-African orogen in East Africa, but this may well represent the limit of deformation within the African plate and could be well west of an actual suture. We would locate the westernmost parts of the Proterozoic island arc west of all occurrences of 900- to 700-m.y.-old diorite but within the area affected by the Pan-African event. The presence of subsidiary or even imbricate zones of subduction within the Arabian Peninsula cannot be discounted, but such zones are inadequately documented at present. Possible locations along the major north-trending shear zones, such as that at about long $42^{\circ} 30^{\prime} \mathrm{E}$., or the major contact between the basaltic and older andesitic assemblage (fig. 3) might be suggested as areas for investigation.

In addition to explaining the early evolution of the present Arabian craton, this model accounts for intraplate deformations subsequent to subduction. Tapponier and Molnar (1976) presented an indentation-deformation model for continental-collision tectonics that has application to the Arabian Peninsula. Similar, though less rigorously developed, collision models have been presented by Sengor (1976), Dewey and Burke (1973), and Dewey and others (1973). During the Pan-African event, the Arabian Peninsula was subjected to simultaneous plutonism, folding, graben formation, and transcurrent faulting with large displacement (Fleck and others, 1976; Delfour, 1970; Brown, 1972; Al-Shanti and Mitchell, 1976). Folding about nearly north-southtrending axes, such as that of 621-m.y.-old aplite dikes in the Al Qarah Quadrangle (loc. 51), must be accommodated by the same tectonic model invoked to explain the northwest-trending Najd fault system. We suggest that a continental-collision model such as that proposed for the Himalayan deformation by Tapponnier and Molnar (1976) provides a unifying explanation of Arabian intraplate tectonics. As shown by those workers, left-lateral strike-slip faults of large displacement, such as the Altyn Tagh fault north of the Himalayas, would be predicted by analogy to indentation of a semiinfinite rigid-plastic medium (Asia) by a rigid indenter (India). The angular relations of the northwest-trending Najd fault system to the nearly north-south trend of compressional structures in Saudi Arabia are nearly identical to those between the Altyn Tagh fault and the Himalayan fold system (fig. 30). These collisionlike intraplate tectonics combined with the strong evidence of pre-PanAfrican isolation from continental areas, the chemiical evidence cited by Greenwood and Brown (1973) and Greenwood and others (1976) for an eastdipping subduction zone, and the extended period of eastward-migrating, subduction-related magmatism suggest that the Pan-African event represents the culmination of orogenesis in Arabia during which time the juvenile Arabian subcontinent or island arc was sutured to the Proterozoic African Continent. This last tectonic episode probably extended from before 650 m.y. to at least 540 m.y. ago, and the cooling of rocks now exposed was completed after about 520 m.y. ago. 

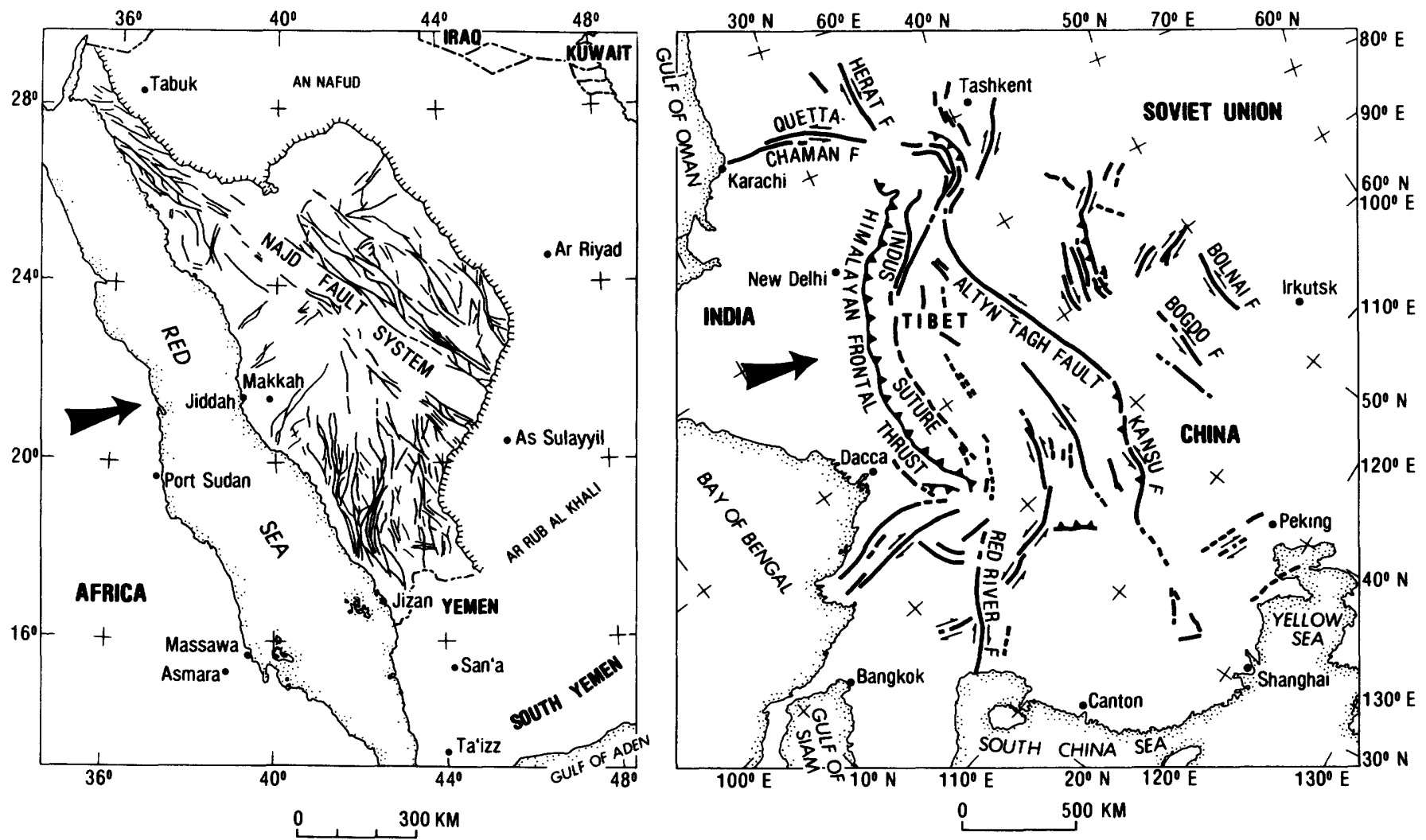

Figure 30.-A comparison of continental-collision-related structures of Asia (after Tapponníer and Molnar, 1976) with orogenic structures of the Arabian Shield. Large arrows indicate implied direction of subduction prior to collision.

\section{CONCLUSIONS}

Basaltic volcanism with island arc affinities rather than mid-ocean-rift affinities occurred prior to 1,100 m.y. ago in the area presently incorporated into the Arabian Shield. Island-arc volcanism, sedimentation, and plutonism began prior to $900 \mathrm{~m} . \mathrm{y}$. ago; magmas were dioritic to trondhjemitic, probably derived by partial melting of oceanic lithosphere or, less probably, upper mantle. After about 775 m.y. ago, more alkali-rich melts appeared with quartz diorite-granodiorite replacing diorite-trondhjemite. The youngest dioritic units occur in the eastern parts of the shield, emplaced between 680 and 720 m.y. ago when the north- or northwest-trending zone of magmatism migrated to its most easterly position. After this period of subduction-related orogenesis, called the Hijaz tectonic cycle, the newly formed arc was sutured to the Proterozoic African plate during a collisional phase recognized in Arabia and eastern Africa as the Pan-African orogeny. Plutonism related to this event began between 660 and $640 \mathrm{~m} . \mathrm{y}$. ago and continued after the major deformational period was concluded. The youngest, possibly postorogenic, plutonic rocks of the shield were probably formed between about 610 and 570 m.y. ago, but low-grade metamorphism and wrench-fault tectonism continued until about 520 m.y. ago.

\section{REFERENCES CITED}

Al-Shanti, A. M. S., and Mitchell, A. H. G., 1976, Late Precambrian subduction and collision in the Al Amar-Idsas region, Arabian Shield, Kingdom of Saudi Arabia: Tectonophysics, v. 30, p. T41-T47.

Anderson, R. E.. 1977, Geology of the Wadi Tarj quadrangle, Sheet 19/42A, Kingdom of Saudi Arabia: Saudi Arabian Directorate General Mineral Resources Geologic Map GM-29, scale 1:100,000, with text.

1978a, Geology of the Wadi 'Atf quadrangle, Sheet 17/43A, Kingdom of Saudi Arabia: Saudi Arabian Directorate General Mineral Resources Geologic Map GM-30, scale 1:100,000, with text. - 1978b, Geology of the Mayza quadrangle, Sheet 17/43B, Kingdom of Saudi Arabia: Saudi Arabian Directorate General Mineral Resources Geologic Map GM-31, scale $1: 100,000$, with text.

Bakor, A. R., Gass, I. G., and Neary, C. R., 1976, Jabal al Wask, northwest Saudi Arabia-An Eocambrian backarc ophiolite: Earth and Planetary Science Letters, v. 30 , p. $1-9$.

Beckinsale, R. D., and Gale, N. H., 1969, A reappraisal of the decay constants and branching ratios of ${ }^{40} \mathrm{~K}$ : Earth and Planetary Science Letters, v. 6, p. 289-294. 
Bramkamp, R. A., Brown, G. F., Holm, D. A., and Layne, N. M., Jr., 1963a, Geologic map of the Wadi as Sirhan quadrangle, Kingdom of Saudi Arabia: U.S. Geological Survey Miscellaneous Geologic Investigations Map I-200A, scale $1: 500,000$.

Bramkamp, R. A., Ramirez, L. F., Brown, G. F., and Pocock, A. E., 1963b, Geologic map of the Wadi ar Rimah quadrangle, Kingdom of Saudi Arabia: U.S. Geological Survey Miscellaneous Geologic Investigations Map I-206A, scale $1: 500,000$.

Brooks, C., Hart, S. R., Hofman, A., and James, D. E., 1976, $\mathrm{Rb}-\mathrm{Sr}$ mantle isochrons from oceanic regions: Earth and Planetary Science Letters, v. 32, p. 51-61.

Brooks, C., Hart, S. R., and Wendt, I., 1972, Realistic use of two-error regression treatments as applied to rubidiumstrontium data: Reviews of Geophysics and Space Physics, v. 10 , p. $551-577$.

Brown, G. F., 1970, Eastern margin of the Red Sea and the coastal structures in Saudi Arabia: Royal Society of London Philosphical Transactions, v. A267, p. 76-87.

1972, Tectonic map of the Arabian Peninsula: Saudi Arabian Directorate General Mineral Resources Arabian Peninsula Map AP-2, scale 1:4,000,000.

Brown, G. F., and Jackson, R. O., 1960, The Arabian Shield: International Geological Congress, 21st, Copenhagen 1960, Proceedings, sec. 9, p. 69-77.

Brown, G. F., Jackson, R. O. Bogue, R. G., and Elberg, E. L., Jr., 1963a, Geologic map of the northwestern Hijaz quadrangle, Kingdom of Saudi Arabia: U.S. Geological Survey Miscellaneous Geologic Investigations Map I-204A, scale $1: 500,000$.

Brown, G. F., Jackson, R. O., Bogue, R. G., and McLean, W. H., 1963b, Geologic map of the southern Hijaz quadrangle, Kingdom of Saudi Arabia: U.S. Geological Survey Miscellaneous Geologic Investigations Map I-210A, scale $1: 500,000$.

Catanzaro, E. J., Murphy, T. J., Garner, E. L., and Shields, W. R., 1969, Absolute isotopic abundance ratio and atomic weight of terrestrial rubidium: National Bureau of Standards Journal of Research, v. 73A, p. 511-516.

Clifford, T. N., 1967, The Damaran episode in the Upper Proterozoic-Lower Paleozoic structural history of southern Africa: Geological Society of America Special Paper 92, $100 \mathrm{p}$.

1968, Radiometric dating and the pre-Silurian geology of Africa, in Hamilton, E. I., and Farquhar, R. H., eds., Radiometric dating for geologists: London, Interscience, p. 299-416.

1970, The structural framework of Africa, in Clifford, T. N., and Gass, I. G., eds, African magmatism and tectonics: Darien, Conn., Hafner, p. 1-26.

Coleman, R. G., 1973a, Reconnaissance geology of the Khamis Mushayt quadrangle, Kingdom of Saudi Arabia: Saudi Arabian Directorate General Mineral Resources Geologic Map GM-5, scale 1:100,000.

- 1973b, Reconnaissance geology of the Khaybar quadrangle, Kingdom of Saudi Arabia: Saudi Arabian Directorate General Mineral Resources Geologic Map GM-4, scale $1: 100,000$.

Coleman, R. G., Brown, G. F., and Keith, T. E. C., 1972, Layered gabbros in southwest Saudi Arabia: U.S. Geological Survey Professional Paper 800-D, p. D143D150.
Coleman, R. G., Ghent, E. D., Fleck, R. J., and Griscom, A., 1973, The Jabal Shayi layered gabbro in southwestern Saudi Arabia: U.S. Geological Survey Saudi Arabian Project Open-file Report 159, 93 p.

Darracott, B. W., 1972, Gravity surveys to delineate African orogenic belts: Nature, v. 240, p. 403-404.

Delfour, Jacques, 1970, Le Groupe de J'Balah, une nouvelle unit du bouclier arabe (The J'Balah Group, a new unit of the Arabian Shield): [France] Bureau Recherches Géologique et Minières Bulletin (ser. 2), sec. 4, no. 4, p. 19-32.

Dewey, J. F., and Burke, K. C. A., 1973, Tibetan, Variscan, and Precambrian basement reactivation-Products of continental collision: Journal of Geology, v. 81, p. 683-692.

Dewey, J. F., Pitman, W. C., III, Ryan, W. B. F., and Bonnin, Jean, 1973, Plate tectonics and the evolution of the Alpine system: Geological Society of America Bulletin, v. 84, p. 3137-3180.

Dick nson, W. R., 1970, Relations of andesites, granites, and derivation sandstones to arc-trench tectonics: Reviews of Geophysics and Space Physics, v. 8, p. 813-860.

Dodge, F. C. W., and Rossman, D. L., 1975, Mineralization in the Wadi Qatan area, Kingdom of Saudi Arabia: U.S. Geological Survey Saudi Arabian Project Open-file Report 190, $71 \mathrm{p}$.

Faure, G., and Powell, J. L., 1972, Strontium isotope geology: Berlin, Springer-Verlag, 188 p.

Fleck, R. J., 1972, Geachronology of late Precambrian and early Paleozoic rocks from Saudi Arabia [abs.]: American Geophysical Union Transactions, v. 43, p. 1130.

Fleck, R. J., Coleman, R. G., Cornwall, H. R., Greenwood, W. R., Hadley, D. G., Prinz, W. C., Ratte, J. S., and Schmidt, D. L., 1976, Potassium-argon geochronology of the Arabian Shield, Kingdom of Saudi Arabia: Geological Society of America Bulletin, v. 87, p. 9-21.

Fleck, R. J., Greenwood, W. R., Hadley, D. G., and Prinz, W. C., 1973, Age and origin of tonalite-granodiorite gneisses, western Saudi Arabia [abs.]: American Geophysical Union Transactions, v. 54, p. 1226.

Garner, E. L., Murphy, T. J., Gramlich, J. W., Paulsen, P. J., and Barnes, I. L., 1975, Absolute isotopic abundance ratios and the atomic weight of a reference sample of potassium: National Bureau of Standards Journal of Research, v. $79 \mathrm{~A}$, no. 6 , p. 713-725.

Gast, P. W., 1967, Isotope geochemistry of volcanic rocks, in Hess, H. H., and Poldervaart, A., eds., Basalts-The Poldervaart treatise on rocks of basaltic composition: New York, Interscience, v. 1, p. 325-358.

Greenwood, W. R., 1975a, Geology of the Biljurshi quadrangle, Kingdom of Saudi Arabia, with a section on Economic geology, by V. A. Trent and T. H. Kiilsgaard, and a section on Aeromagnetic Studies, by G. E. Andreasen: Saudi Arabian Directorate General Mineral Resources Geologic Map GM-25, scale 1:100,000, with text.

$1975 \mathrm{~b}$, Geology of the Al Aqiq quadrangle, Kingdom of Saudi Arabia: Saudi Arabian Directorate General Mineral Resources Geologic Map GM-23, scale 1:100,000, with text.

1979a, Geology of the An Nimas quadrangle, Kingdom of Saudi Arabia: Saudi Arabian Directorate General Mineral Resources Geologic Map, scale 1:100,000, with text [in press]. 
Greenwood, W. R., 1979b, Geology of the Khadrah quadrangle, Sheet 19/42D, Kingdom of Saudi Arabia: Saudi Arabian Directorate General Mineral Resources Geologic Map, scale $1: 100,000$, with text [in press].

$1979 \mathrm{c}$, Geology of the Wadi Wassat quadrangle, Sheet 18/44C, Kingdom of Saudi Arabia: Saudi Arabian Directorate General Mineral Resources Geologic Map, scale $1: 100,000$, with text [in press].

$1979 \mathrm{~d}$, Geology of the Wadi Malahah quadrangle, Sheet 18/43D, Kingdom of Saudi Arabia: Saudi Arabian Directorate General Mineral Resources Geologic Map, scale $1: 100,000$, with text [in press].

Greenwood, W. R., Anderson, R. E., Fleck, R. J., and Roberts, R. J., 1977, Precambrian geologic history and plate tectonic evolution of the Arabian Shield: U.S. Geological Survey Saudi Arabian Project Report 222, $97 \mathrm{p}$.

Greenwood, W. R., and Brown, G. F., 1973, Petrology and chemical analyses of selected plutonic rocks from the Arabian Shield, Kingdom of Saudi Arabia: Saudi Arabian Directorate General Mineral Resources Bulletin $9,9 \mathrm{p}$.

Greenwood, W. R., Hadley, D. G., Anderson, R. E., Fleck R. J., and Schmidt, D. L., 1976, Proterozoic cratonization in southwestern Saudi Arabia: Royal Society of London Philosophical Transactions, ser. A, v. 280, p. 517-527.

Greenwood, W. R., Hadley, D. G., and Schmidt, D. L., 1973, Tectono-stratigraphic subdivision of Precambrian rocks in the southern part of the Arabian Shield: Geological Society of America Abstracts with Programs, v. 5, no. 7, p. 643.

Greenwood, W. R., Roberts, R. J., and Bagdady, Abdulaziz, 1974, Mineral belts in western Saudi Arabia: U.S. Geological Survey Saudi Arabian Project Report 177, $26 \mathrm{p}$.

Hadley, D. G., 1974, The taphrogeosynclinal Jubaylah Group in the Mashhad area, northwestern Hijaz, Kingdom of Saudi Arabia: Saudi Arabian Direcorate General Mineral Resources Bulletin 10, $18 \mathrm{p}$.

1976, Geology of the Bi'r Juqjuq quadrangle, Sheet 21/43D, Kingdom of Saudi Arabia: Saudi Arabian Directorate General Mineral Resources Geologic Map GM-26, scale $1: 100,000$, with text.

Hart, S. R.; Brooks, C., Krogh, T. E., Davis, G. L., and Nava, D., 1970, Ancient and modern volcanic rocks-A trace element model: Earth and Planetary Science Letters, v. 10, p. 17-28.

Holmes, Arthur, 1965, Principles of physical geology (revised ed.) : New York, Ronald Press, $1288 \mathrm{p}$.

Jackson, R. O., Bogue, R. G., Brown, G. F., and Gierhart, R. D., 1963, Geologic map of the southern Najd quadrangle, Kingdom of Saudi Arabia: U.S. Gealogical Sur- vey Miscellaneous Geologic Investigations Map I-211A, scale $1: 500,000$.

Kennedy, W. Q., 1964, The structural differentiation of Africa in the Pan-African ( \pm 500 m.y.) tectonic episode: Leeds University Research Institute of African Geology 8th Annual Report on Scientific Results, p. 48-49.

Kistler, R. W., and Peterman, Z. E., 1973, Variation in Sr, $\mathrm{Rb}, \mathrm{K}, \mathrm{Na}$, and initial ${ }^{87} \mathrm{Sr} /{ }^{88} \mathrm{Sr}$ in Mesozoic granitic rocks and intruded wall rocks in central California: Geological Society of America Bulletin, v. 84, p. 3489-3512.

Lenz, H., Bender, F., Besang, C., Harre, W., Kreuzer, H., Müller, P. and Wendt, I., 1973, The age of early tectonic events in the zone of the Jordan geosuture based on radiometric data: Fortschritte der Mineralogie, v. 50, no. 3, p. 98 .

McIntyre, G. A., Brooks, C., Compston, W., and Turek, A., 1966, The statistical assessment of $\mathrm{Rb}-\mathrm{Sr}$ isochrons: Journal of Geophysical Research, v. 71, p. 5459-5468.

Neary, C. R., Gass, I. G., and Cavanaugh, B. J., 1976, Granitic association of northeastern Sudan: Geological Society of America Bulletin, v. 87, p. 1501-1512.

Neumann, W., and Huster, E., 1976, Discussion of the ${ }^{87} \mathrm{Rb}$ half-life determined by absolute counting: Earth and Planetary Science Letters, v. 33, p. 277-288.

Nier, A. O., 1938, Isotopic constitution of $\mathrm{Sr}, \mathrm{Ba}, \mathrm{Bi}, \mathrm{Tl}$, and Hg: Physics Review, v. 54, p. 275-278.

Norrish, K., and Chappell, B. W., 1967, X-ray fluorescence spectrography, in Zussman, J., ed., Physical methods in determinative mineralogy: London, England, Academic Press, p. 161-214.

Peterman, Z. E., and Hedge, C. E., 1971, Related strontium isotopic and chemical variations in oceanic basalts: Geological Society of America Bulletin, v. 82, p. 493-500.

Roddick, J. C., 1978, The application of isochron diagrams in ${ }^{40} \mathrm{Ar}-{ }^{30} \mathrm{Ar}$ dating-a discussion: Earth and Planetary Science Letters, v. 41, p. 233-244.

Schmidt, D. L., Hadley, D. G., Greenwood, W. R., Gonzalez, Louis, Coleman, R. G., and Brown, G. F., 1973, Stratigraphy and tectonism of the southern part of the Precambrian Shield of Saudi Arabia: Saudi Arabian Directorate General Mineral Resources Bulletin, v. 8, 13 p.

Sengor, A. M. C., 1976, Collision of irregular continental margins: Implications for foreland deformation of alpinetype orogens: Geology, v. 4, p. 779-782.

Tapponnier, P., and Molnar, P., 1976, Slip-line field theory and large-scale continental tectonics: Nature, v. 264, p. 319-324.

York, D., 1969, Least-squares fitting of a straight line with correlated errors: Earth and Planetary Science Letters, v. 5 , p. $320-324$. 
